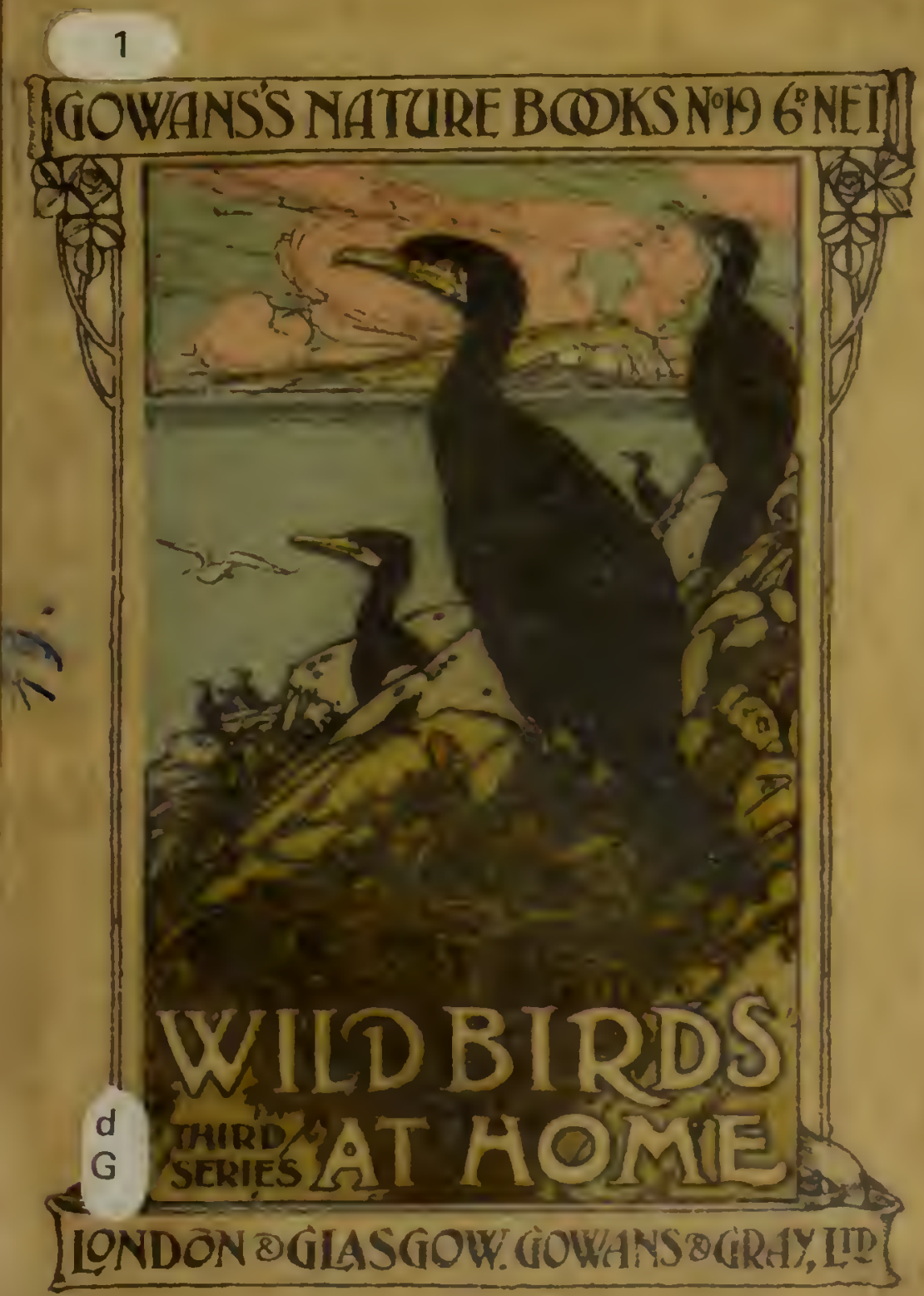




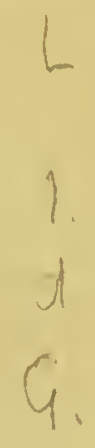

Presented by the Executors of Norman Douglas Simpson I 890 - I974 


\section{Gowans's Nature Books}

$T^{\gamma} \mathrm{HE}$ object of these little books is to stimulate a love for nature and a desire to study it.

All the volunes of the serics that huve been issued so $f a r$ have been very suceessful, and the publishers hope to be able to mintaln the very high standard of excellence which has made this serles so well known all over the country. Some of the photograplis included in the different volumes are uncqualled and unique irlumphs of the naturephotographer's art.

No. 1.-WILD BIRDS AT HOME. Sixty Photo. graphs froin Life, by Chas. Kirk, of British Bird. and their Nests.

No. 2.-WILD FLOWERS AT HOME. First Series. Sixty Photographs from Nature, by Cameron Todd.

No. 3.-WILD FLOWERS AT HOME. Second Series. By the Saine.

No. 4.-BUTTERFLIES AND MOTHS AT HOME. Sixty Plotographs from Life, by A. Forrester.

No. 5.-WILD BIRDS AT HOME. Second Series. By Chas. Kirk.

No. 6. FRESHWATER FISHES. Sixty Photographs from Life, by Walford B. Johnson and Stanley C. Johnson, M.A.

No. 7.-TOADSTOOLS AT HOME. Sixty Photographs of Fungi, by Somerville Hastings, F.R.C.S.

No. 8. OUR TREES \& HOW TO KNOW THEM. Sixty Photographs by Chas. Kirk. 
No. 9. -WILD FLOWERS AT HOME, Third Series. By Somerville Hastings, F.R.C.S.

No. I0.-LIFE IN THE ANTARCTIC. Sixty Photographs from Life by Members of the Scottish National Antarctic Expedition.

No. Ix.-REPTILE LIFE. Sixty Photographs from Life, by Walford B. Johnson and Stanley $C$. Johnson, M.A.

No. 12.-SEA-SHORE LIFE. Sixty Photographs by the Same.

No. 13.-BIRDS AT THE ZOO. Sixty Photographs from Life, by W.S. Berridge, F.Z.S.

No. 14.-ANIMALS AT THE ZOO. Sixty Photographs by the Same.

No. 15.-SOME MOTHS AND BUTTERFLIES AND THEIR EGGS. Sixty Photographs by A. E. Tonge, F.E.S.

No. 16.-WILD FLOWERS AT HOME. Fourth Series. By Somerville Hasting .

No. 17.-BRITISH MAMMALS. Sixty Photographs from Life, by Oxley Grabham, M.A., T. A. Metcalfe, Sydney H. Smith, and Chas. Kirk.

No. 18.-POND AND STREAM LIFE. Sixty Photographs from Life, by Walford $B$. Johnson and Stanley C. Johnson, M.A.

No. 19.-WILD BIRDS AT HOME. Third Series. By Chas. Kirk.

No. 20.-ALPINE PLANTS AT HOME. First Series. Sixty Photographs by Somerville Hastings, F.R.C.S.

\section{Others in Preparation.}

PRice 6d. Net. Each Volume, Postage id. Each.

GOWANS \& GRAY, Ltd, London \& Glasgow

Natural History Museum Library

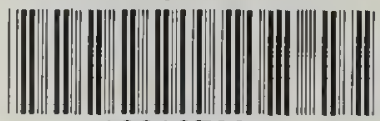

000097230 


\section{The Captive of Love.}

\section{A NATIVE JAPANESE NOVEL}

A strange and fascinating story from the original of Bakin, the greatest of Japadese Novelists.

$$
\text { Price, od. Portage, } 20 .
$$

\section{Robinson's New}

Testament. .

The New Testzment, Authorised Version, rubv type, with 5 s beautful drawings in black and red by Charles Robinson.

$$
\text { CLoth, 28. Net. LEATHER, 20. B. Net. }
$$

$$
\text { postace, zio. }
$$

COWANS'S PRACTICAL PICTURE BOOKS-No. 1.

\section{Ambulance Illustrated,}

B) WM. CULLEN, M.D.

Sirty Photographs by W. M. Warneuke, fllustrat. Ing First Ald, with conclsi notes by the author,

Pice, od. Net poetaez, 10.

jOWANS \& GRAY, Ltd, London and Glasgow 


\section{THE}

\section{Illustrated Nero Testament}

Contains 18 Full Page and 49 Smaller Photographic Views of Holy Places, Two Maps, and an Etched Frontispiece, after

\section{"THE LIGHT OF THE WORLD,"}

BY W. HOLMAN HUNT:

\section{Published in Four Styles.}

o. Cloth LImp (without Frontlsplece), . . Price Net.

1. Art Linen, Red Edges, . - . . . 1s. Od.

2. French Morocco, Gilt Edges, - - - - 18. 6d.

3. Morocco, Red under Gold Edges, - - 2s. Bd. Postago, 11/2d.

\section{The New Illustrated Bible}

Contains 27 Full Page and 103 Smaller Reproductions of Copyright Photographs of places mentioned in the Holy Scriptures; also, Five Maps and

a Photogravure Frontispieco,

\section{"JERUSALEM."}

\section{Published in Three Styles.}

1. Cloth Bourds, Red Edges. - . - . 8s, Odek 1a. Cloth Boards, Gilt Edges, - - - - - 3s. 6d. 2. French Morocco, Red under Gold Edges, - 48. 6d.

a. Morocco, Red under Gold Edges, - - - 6s. Od. Postage, 4d.

GOWANS \& GRAY, Ltd., London and Glasgow. 


\section{THE LIFE OF \\ LOPE \\ $\mathrm{DE}$ \\ VEGA \\ The Great Spanish Dramatist}

BY HUGO A. RENNERT

PROFESSOR OF ROMANIC LANGUAGES AND LITERATIRE IN THE UNIYBRSITY OF PRNNSYLVANIA

THERE has been no life of Lope de Vega published in English since that by Lord Ilolliund nearly 100 years ago.

Professor Rennert has been able to take advantage of the labours of all previous workers in the same field, and las in addition been fortunate atove all his predecessors in that documents were published for the fist time within the last few years by Señor Pérez Pastor, which threw a Hood of light on many dark points in Lope's career.

He has made full use of all fresh data, and has endeavoured to record every known fact in the life of the poet. Il is biography is therefore the most thorough yet published, and in the opinion of Mr. Fitzmaurice-Kelly, the leading English authority, his biography is the standard life. It is consequently indispensable io every important library.

Demy 8vo, Cloth, Gilt Top. Price, 12s. 6r. net

GOWANS \& GRAY, LTD., LONDON AND GLASGOV 


\section{LOPE DE VEGA \& THE SPANISH DRAMA}

BEING THE TAYLORIAN LECTURE (1902)

BY JAMES FITZMAURICE-KELLY

An extremely eloquent essay, of great interest to all students of the drama.

Paper Covers, is. net. Postage, Id.

\section{MRS. MCCRAW}

BY SAMUEL MACPLOWTER, RULING ELDER

The 'Kailyard' as it really is; not the idealised presentment of it as given in the novels of J. M. Barrie and Ian Maclaren. Clever character sketches of a most impudent woman, by a resident somewhere in the Thrums district.

$$
\text { Price, Is. net. Postage, I } \frac{1}{8} d \text {. }
$$

\section{THE BIRTHDAY BOOK OF

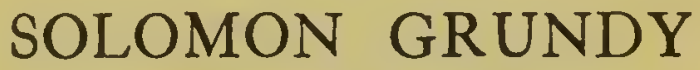

ILLUSTRATIONS BY A. S. BOYD LETTERPRESS BY WILL. ROBERTSON

Scotsmax-'The book is really very funny, and will unquestlon. ably be popular."

Puxch-'Solomon Grundy's Birthday Book's a charmlng publica. tion. Devised by Robertson and Boyd for people's delectation."

Prices, Is. and Is. 6d. Postage, I $\frac{1}{d} d$.

GOWANS \& GRAY, LTD., LONDON AKD GLAscon 


\title{
A TREASURY
}

OF

\section{ENGLISH VERSE}

\author{
SELECTED BT \\ ADAM L. GOWANS, M.A. \\ WITH DECORATIVE TITLE-PAGE AND END-PAPEKS, \\ TRONTISPIKCE, 49 TULL PAGE AND IO3 \\ SMALLER ILLUSTRATIONS, BY \\ STEPHEN REID
}

A most dellghtful volume to hendle; beautifully illustrated and printed

Imperial 16 mo. Art Cloth Gilt, G. Top Price: 5s. met. Pastage, 4d. extra

GOWANS \& GRAY, LTD, LONDON AND GLAgOW 


\section{Great Continental Successes, No. I}

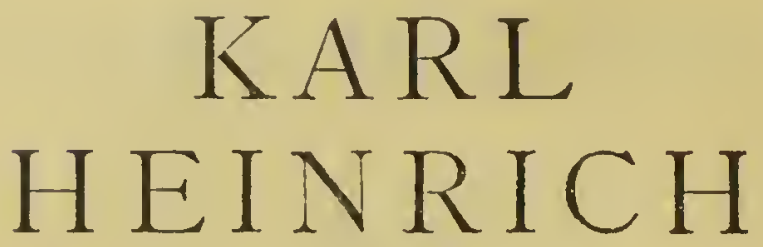

The Novel on which is founded the Play

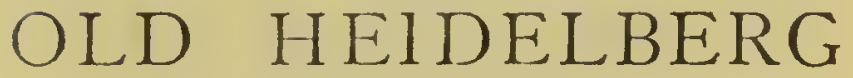

WHICH HAS BEEN GIVEN WITH SUCH VERY GREAT SUCCESS BY MR. GEORGE ALEXANDER

BY W. MEYER-FÖRSTER

WITH I3 ILLUSTRATIONS

THE ONLY TRANSLATION

Cloth, 3s. 6d. net

GOWANS \& GRAY, LTD., London and Glasgow 
Gowanss Nature Books, No. 19

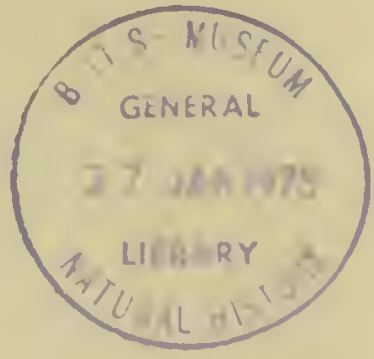

WILD Birds at Home

TIIRD SERIES

CARSON \& NICOL FRINTERS, GLASOOW. 
RUTICILLA PHENICURUS, L.

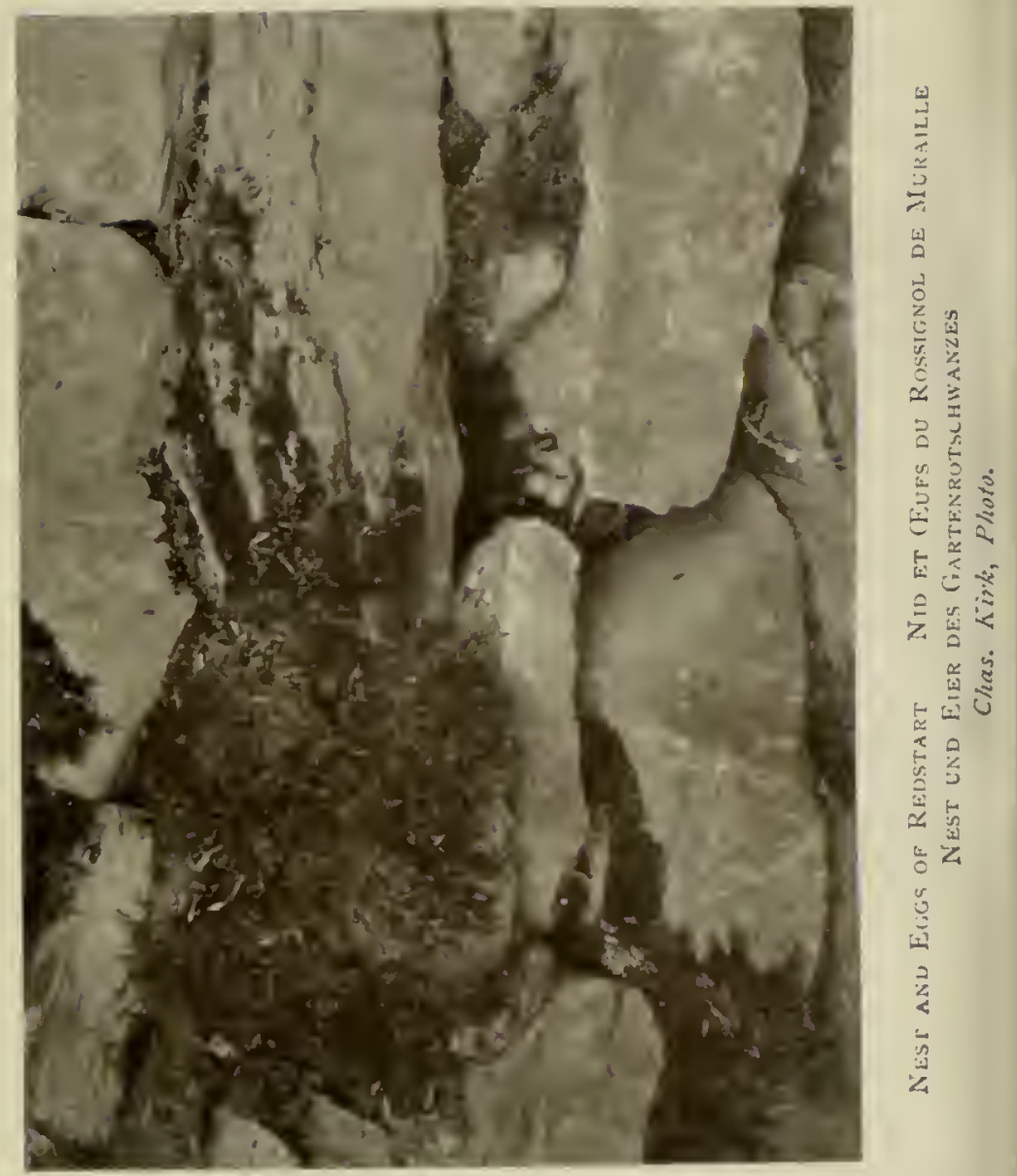



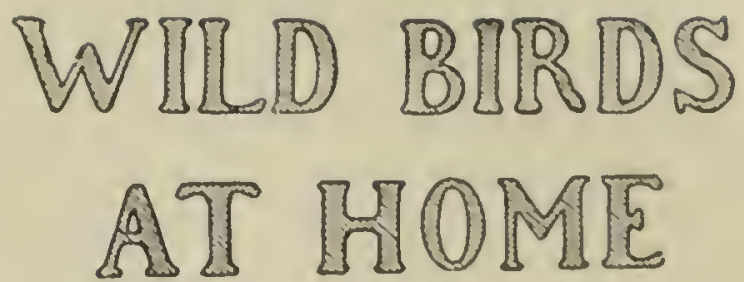

TUKRD SERIES

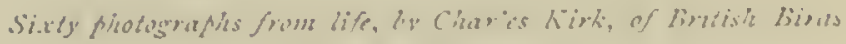
andicin liss

GOW.IXS \& GKAY, LTH.

5 lobert Sireet, Auelph, Loxuor, W.C.

jS Caldogax STREet, Glasgow $190 ;$ 


\section{AGEVTS FOR THE SALE OF TIIS SERIES}

France: A. Perche, t5 Rue Jacob, Paris.

Germany: Hithelm II eicher, Inselstrasse ro p.r., Lcipzig.

Savitzerlant: J. Frankfurer, 12 Grand-Chinc, Lausanue.

Belgium: Spincux \& Cie, 3 Rue du Bois-Suminge, brussels.

Scandinaria: A. So O. Schedin, Lum, Swetin.

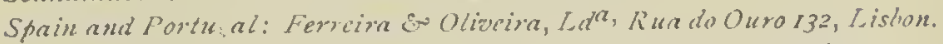
IJolland: Kivbeiger So Kesper and Jacs. C. Kolbers, Amsterdam.

A. Abrahams, The Hiegue.

Ancrica: Dotge Publishing Company, fo-ft East 10th Strect, Nez' l'ork. 


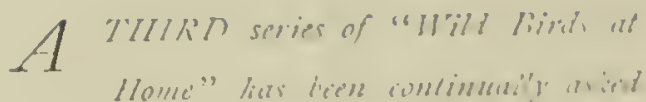

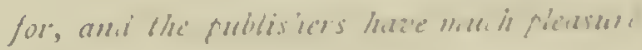

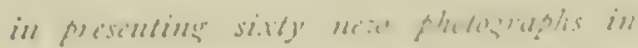

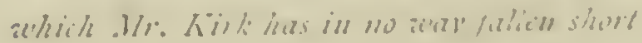

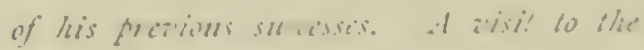

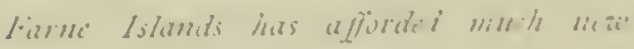

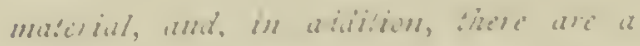

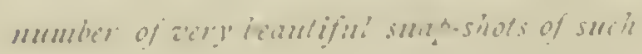
interestins birds as the withet, the snife, and the rinsid fivier. 


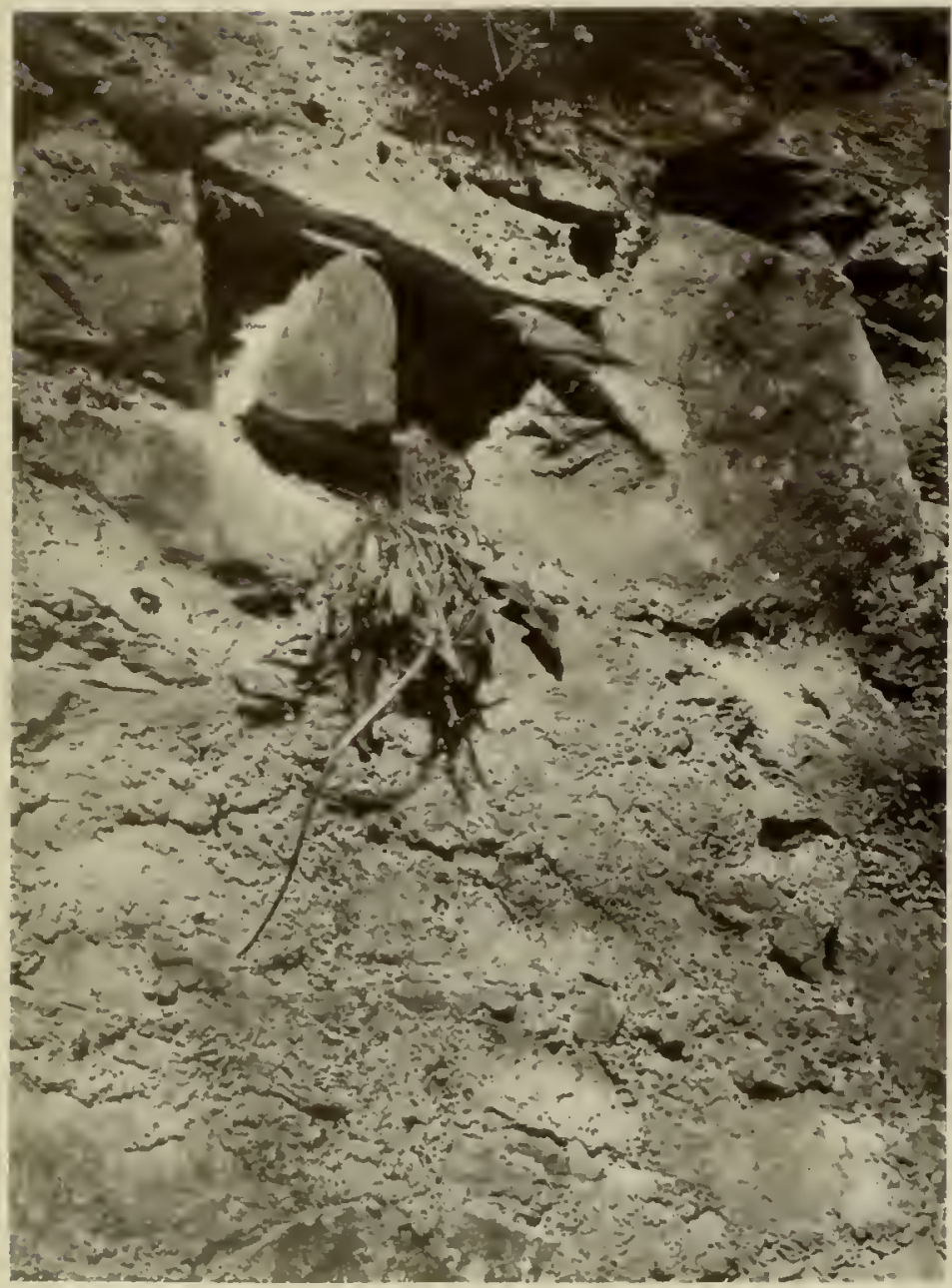

RFISTART AT ENTRANCE OF Nies:
Rossicnot, DE MURAIt.t.E A

L'LNTRFE I)U NIU

Gakllintorscilwan? am Fincianci dis Nhsths

Clus. ん̈ir, Hhoto. 


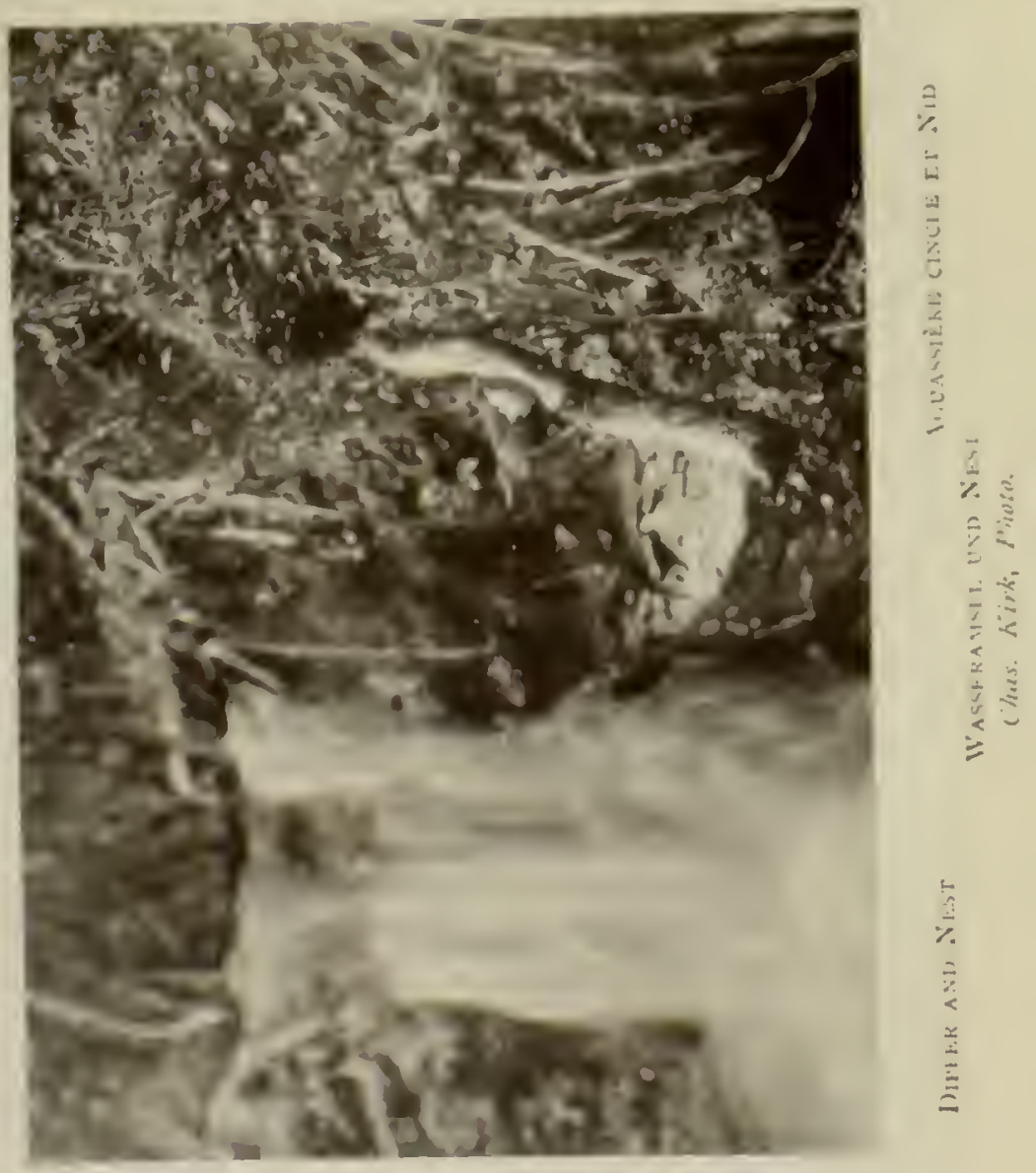




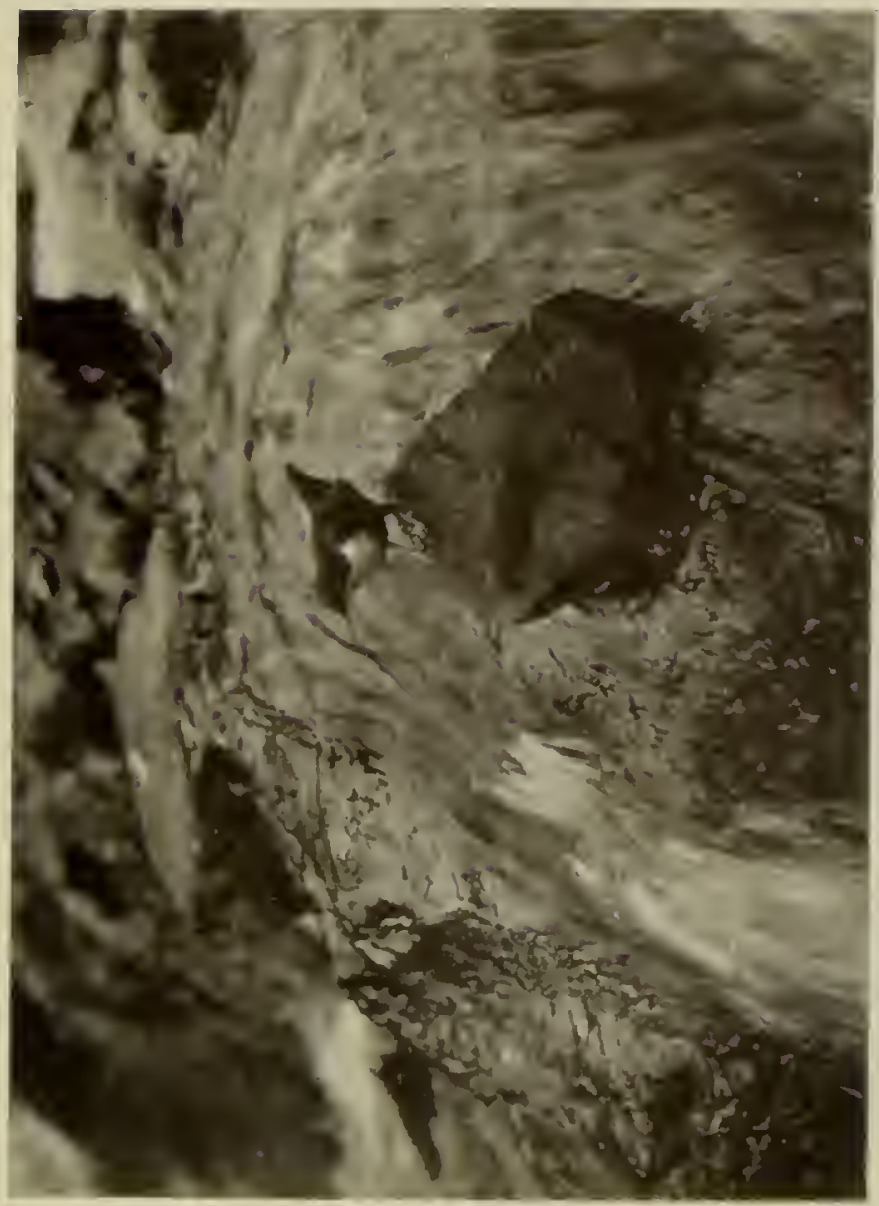

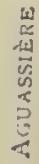

$\frac{1}{4}$ 


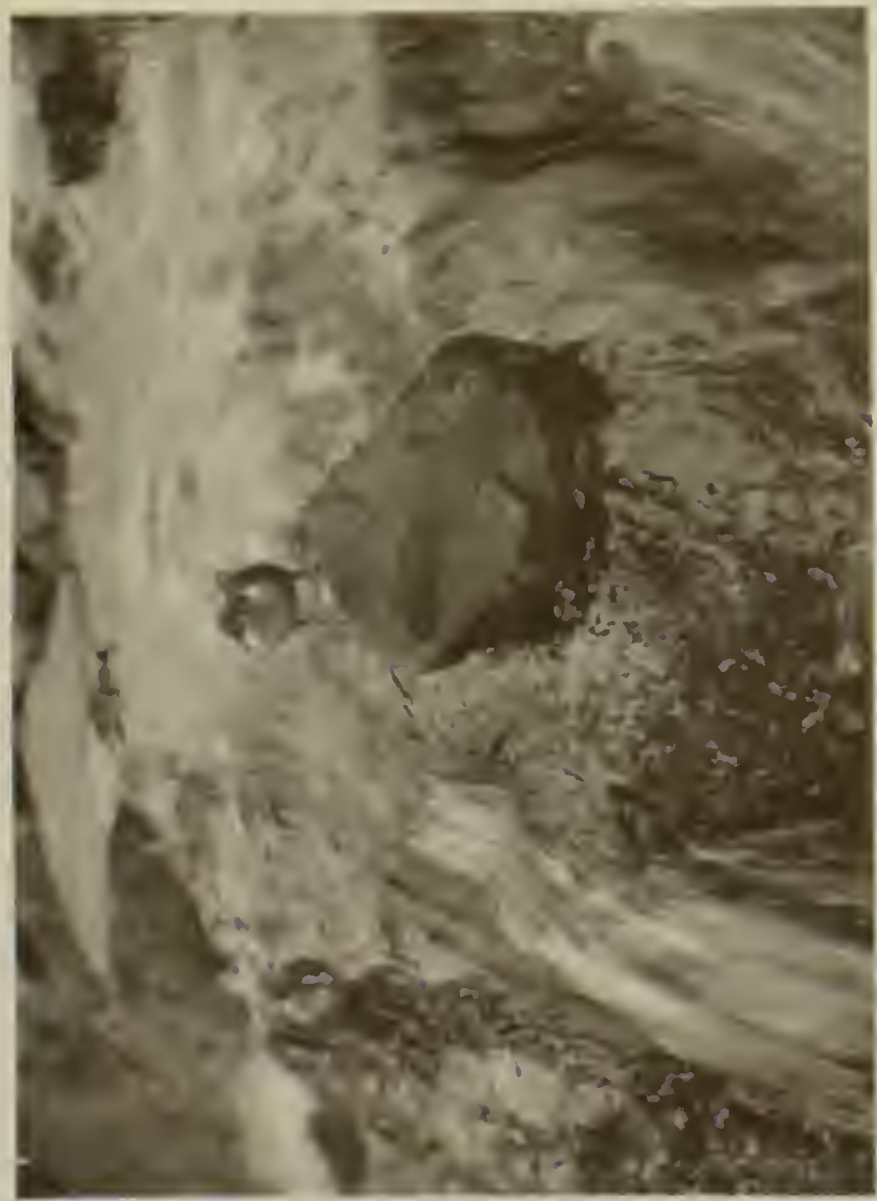

a

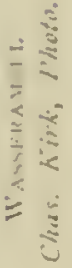

\pm
$=$
$=$ 


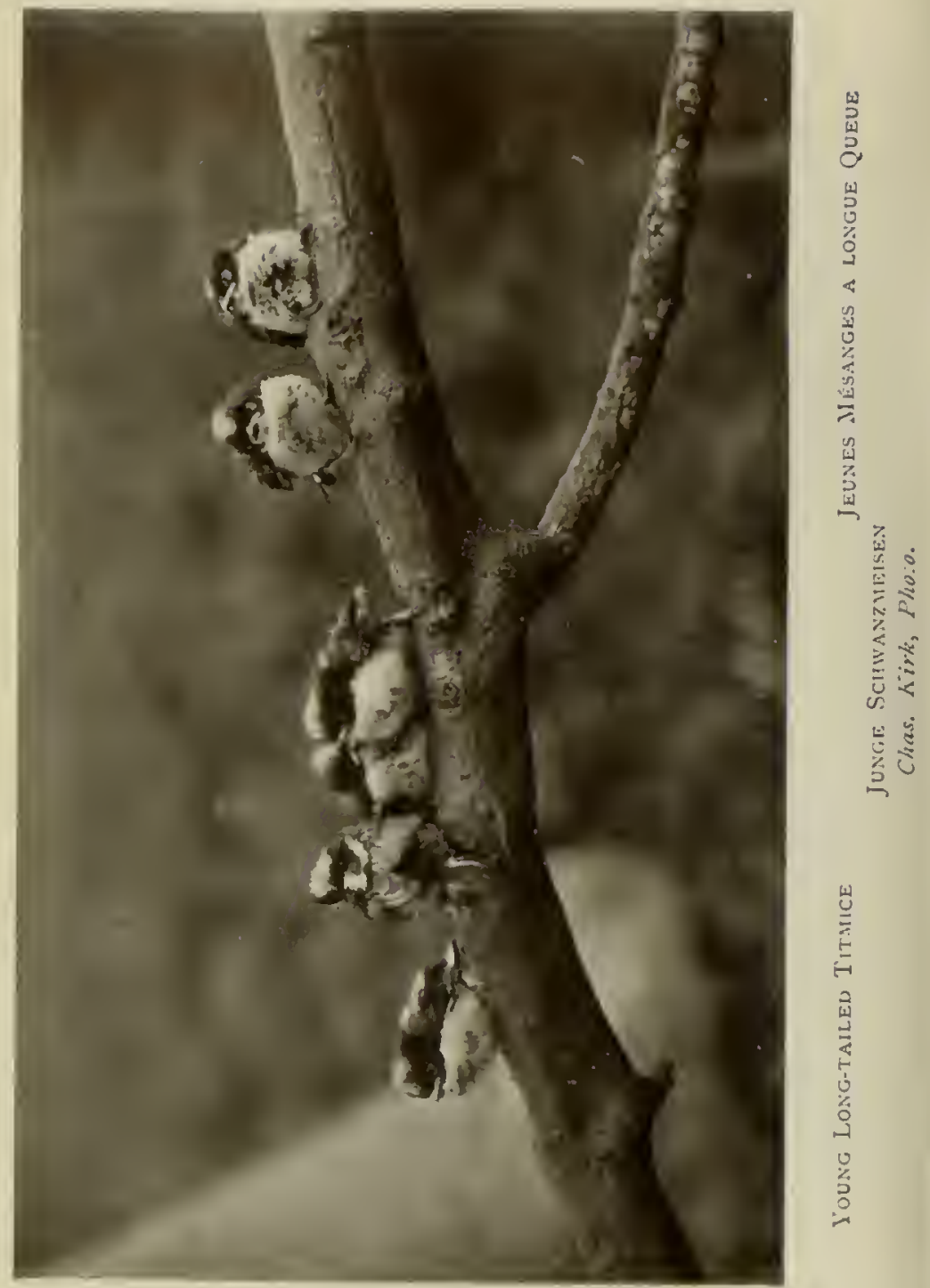




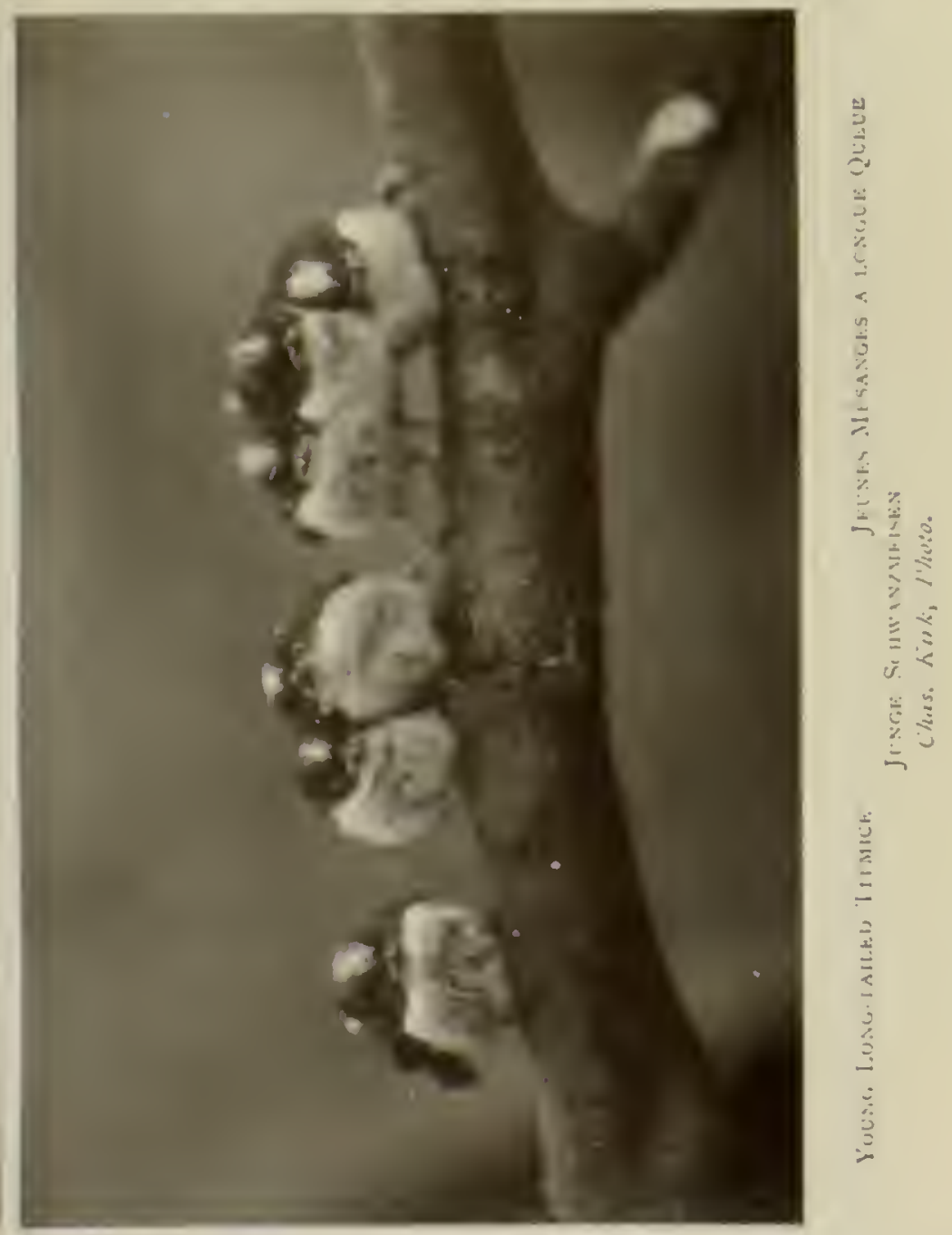




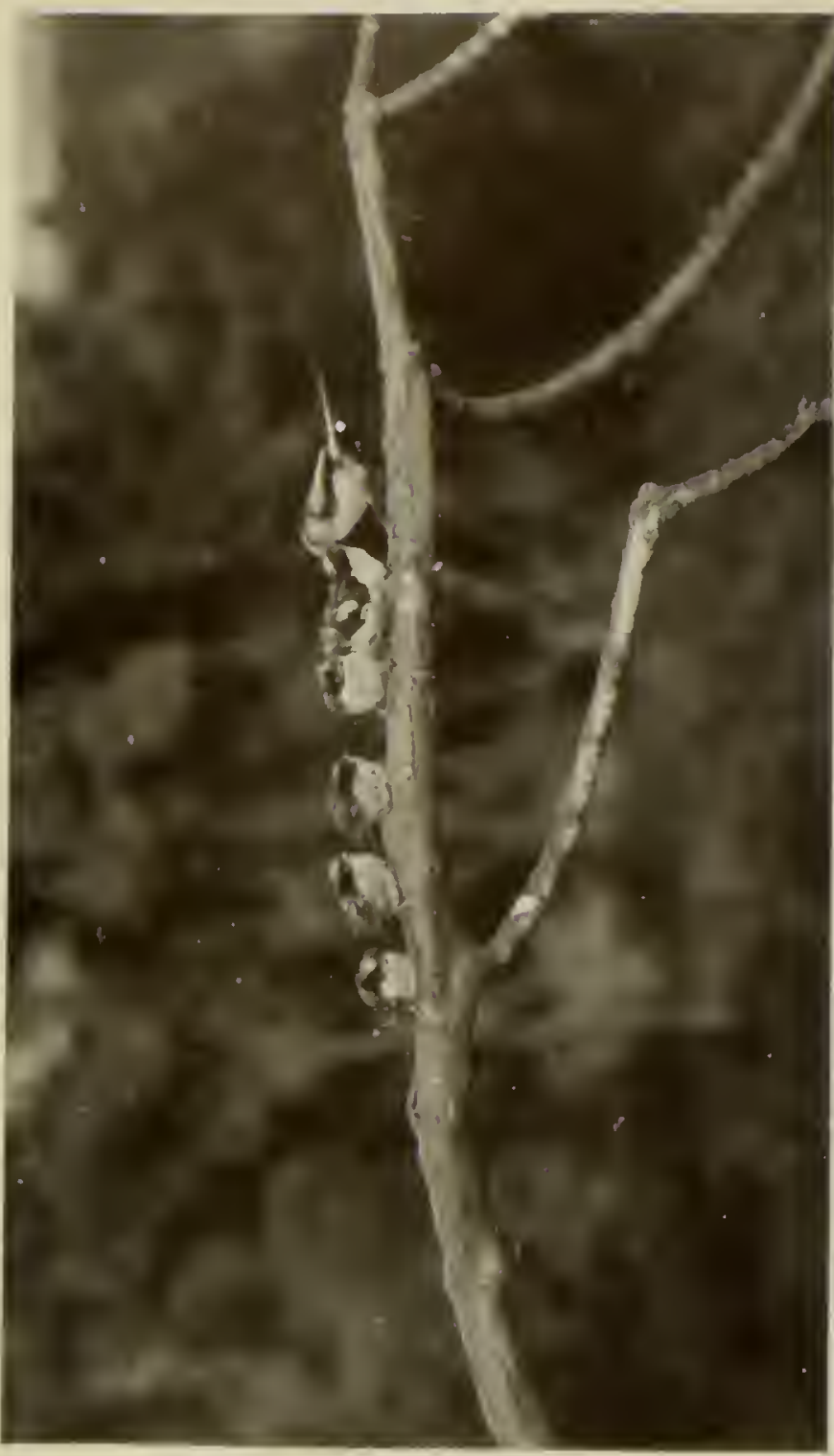




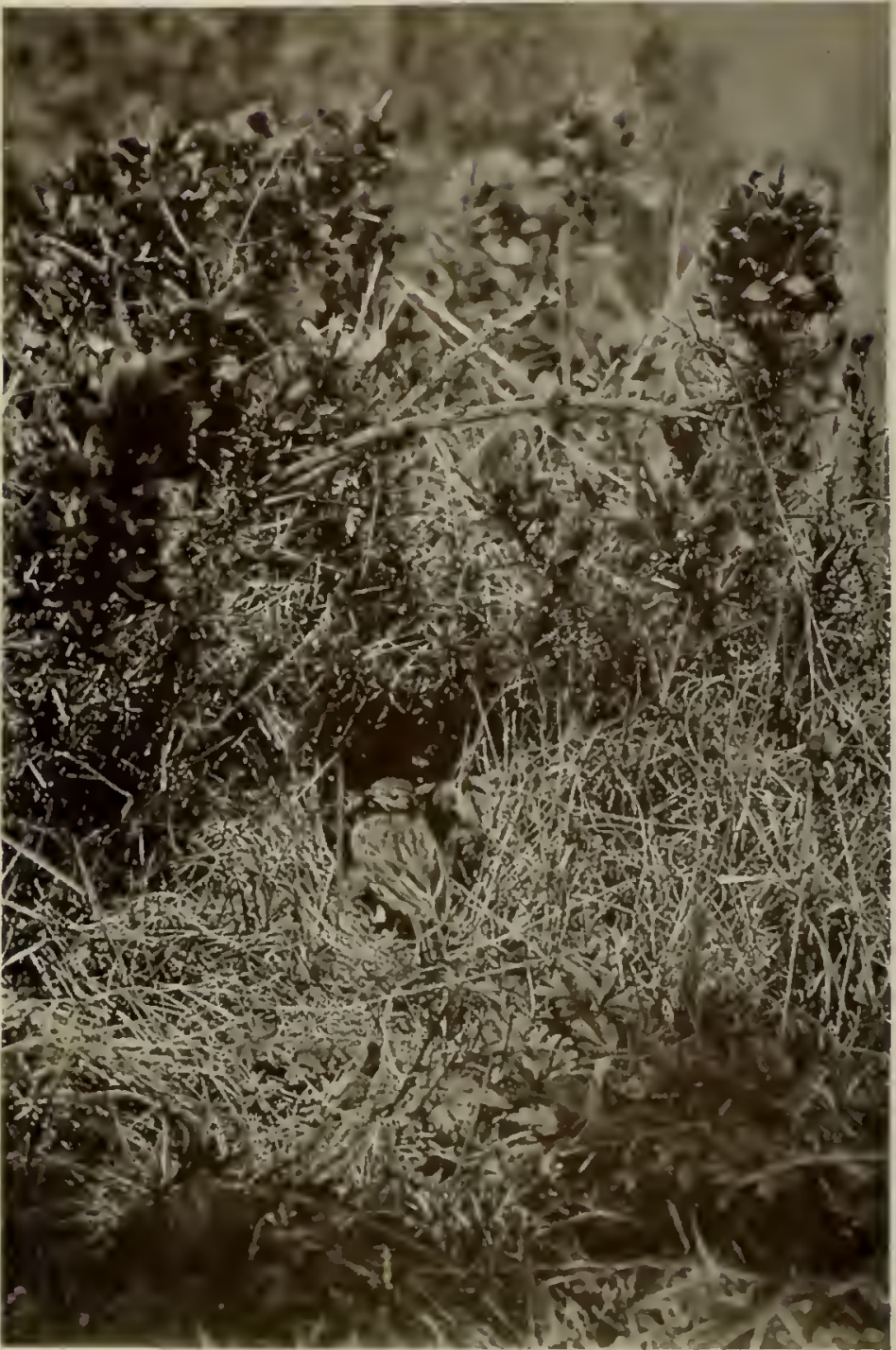

Meadow-Pipit at Entrance Pijl des Prés (Fartoush) a OF NEST L'ENTREE DU NiU

WIESHANIEPER AM EINGANG DES NESTES 


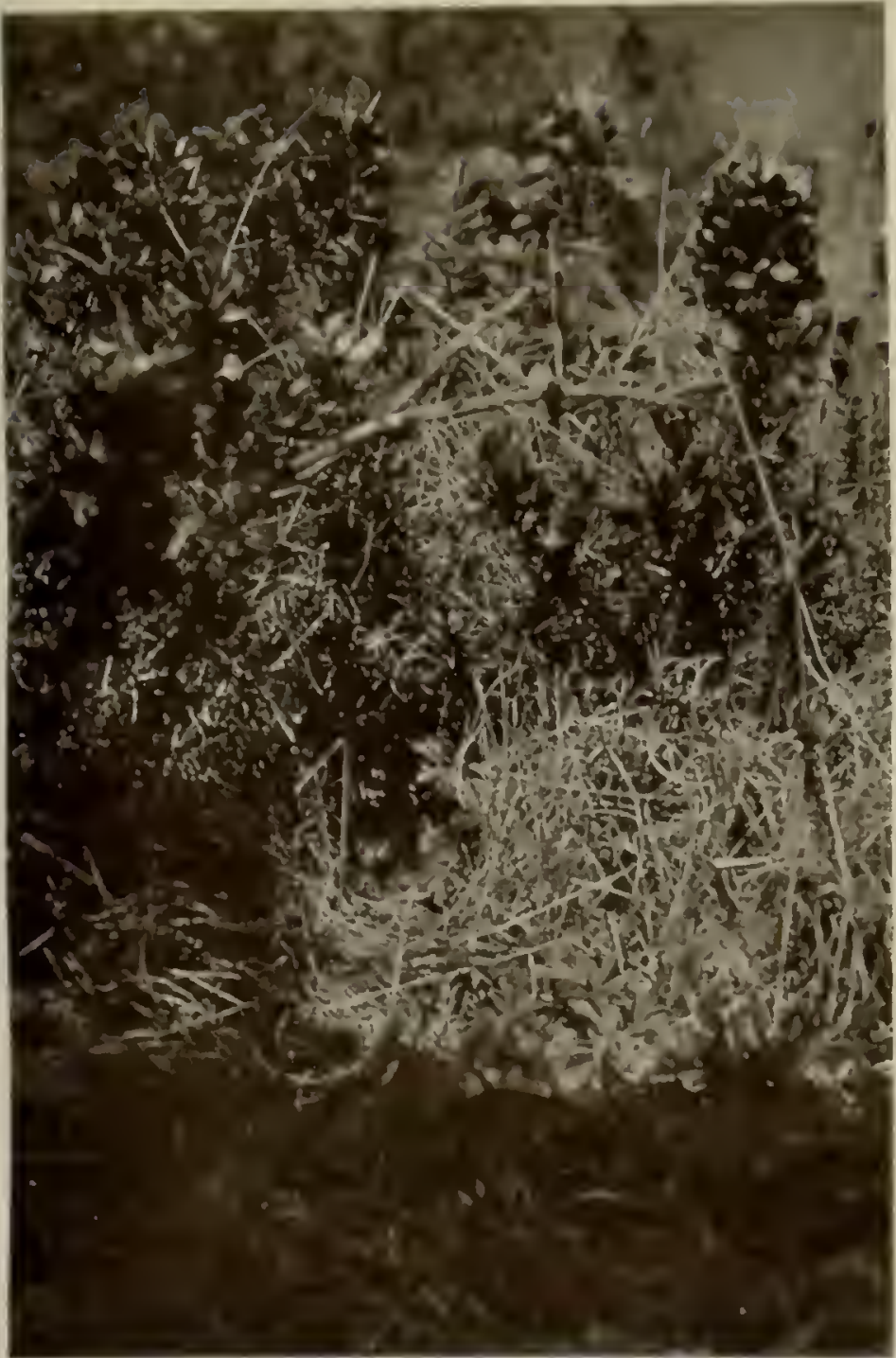

Mr.nuW-PlPt ox Nest

PIII DIES PRÉS SLR NIU

Wiesentifeper auf Nest

Chas. Kini, Pisu. 


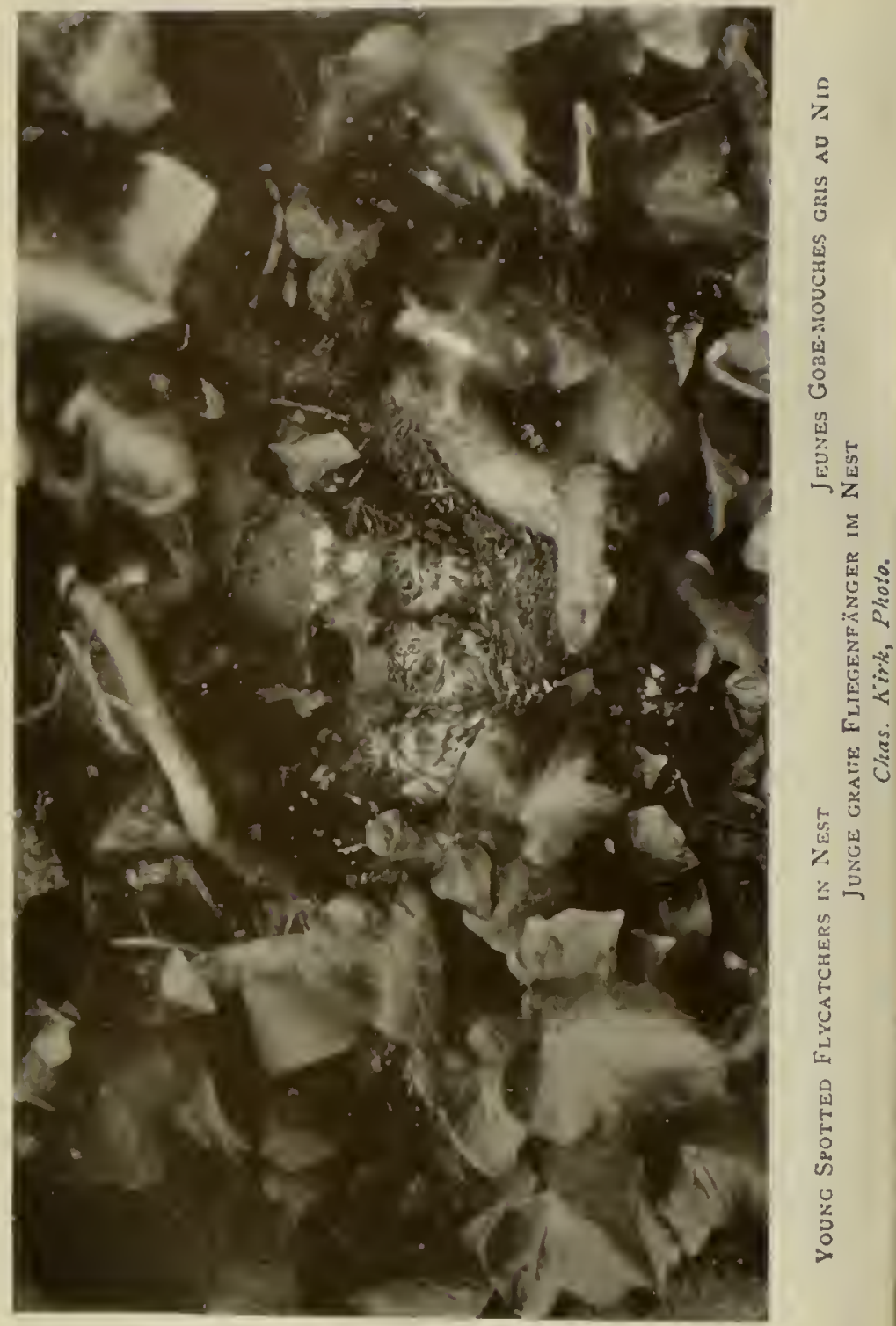




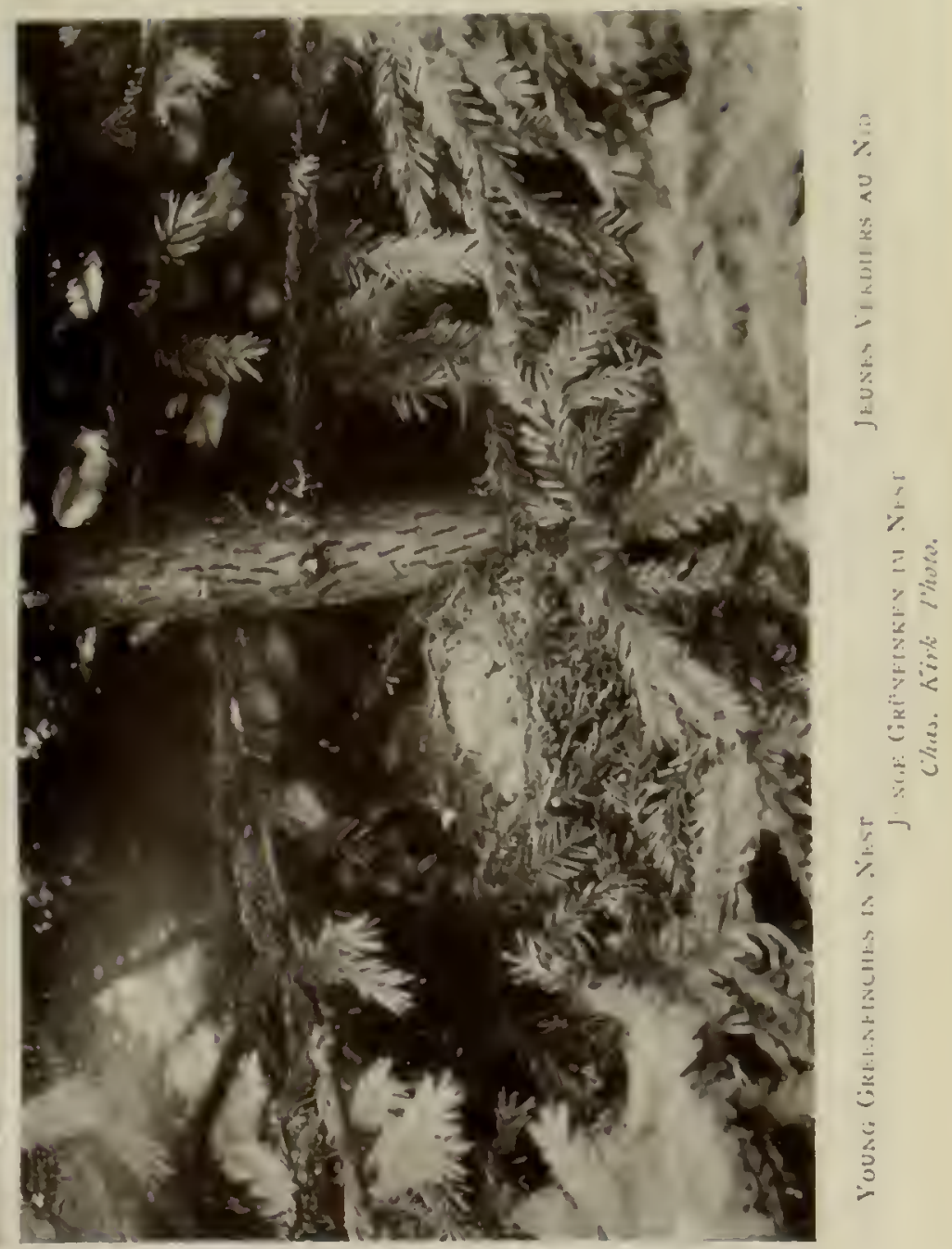




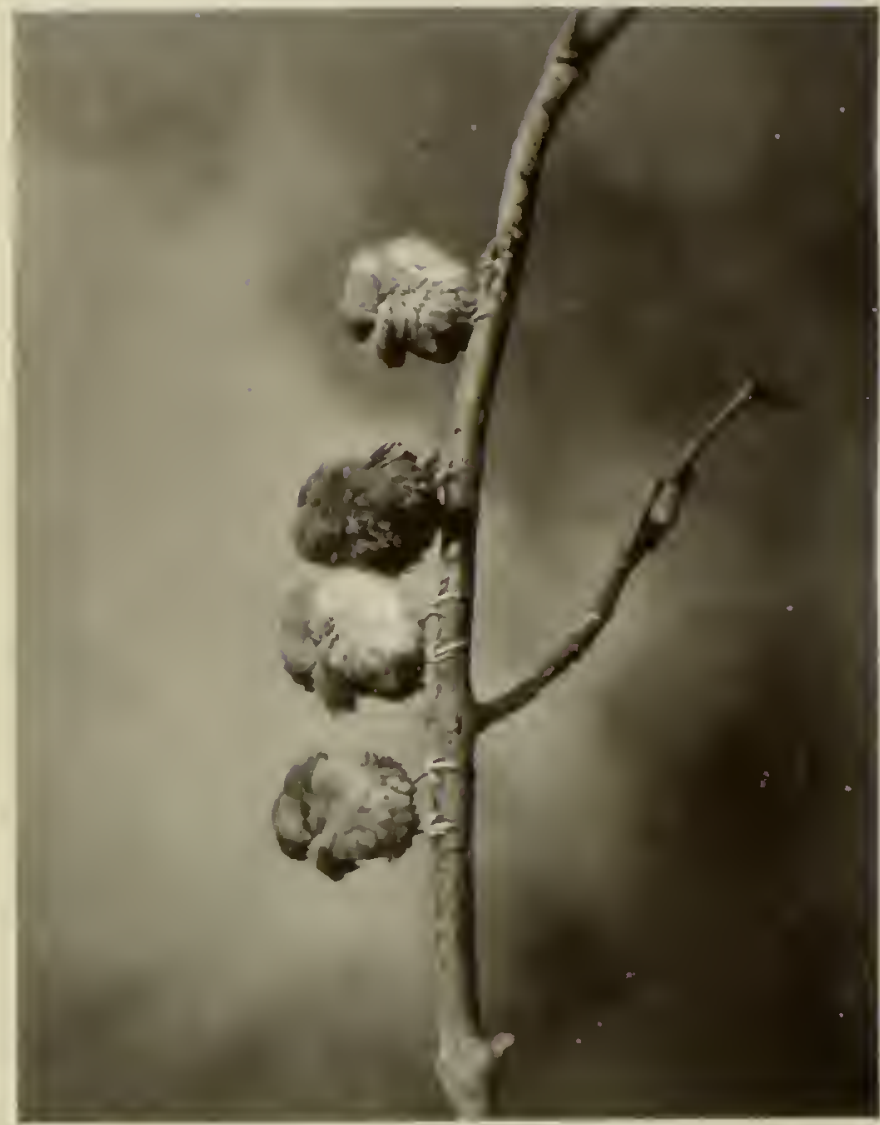

(5)

(5)

(4) $z$

Z

s<

包

z

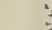

I

()ㅗ․

trot

4 实

इ

4

$=$

$\%$

ta

(4)

5

ช

0
2
3
5 


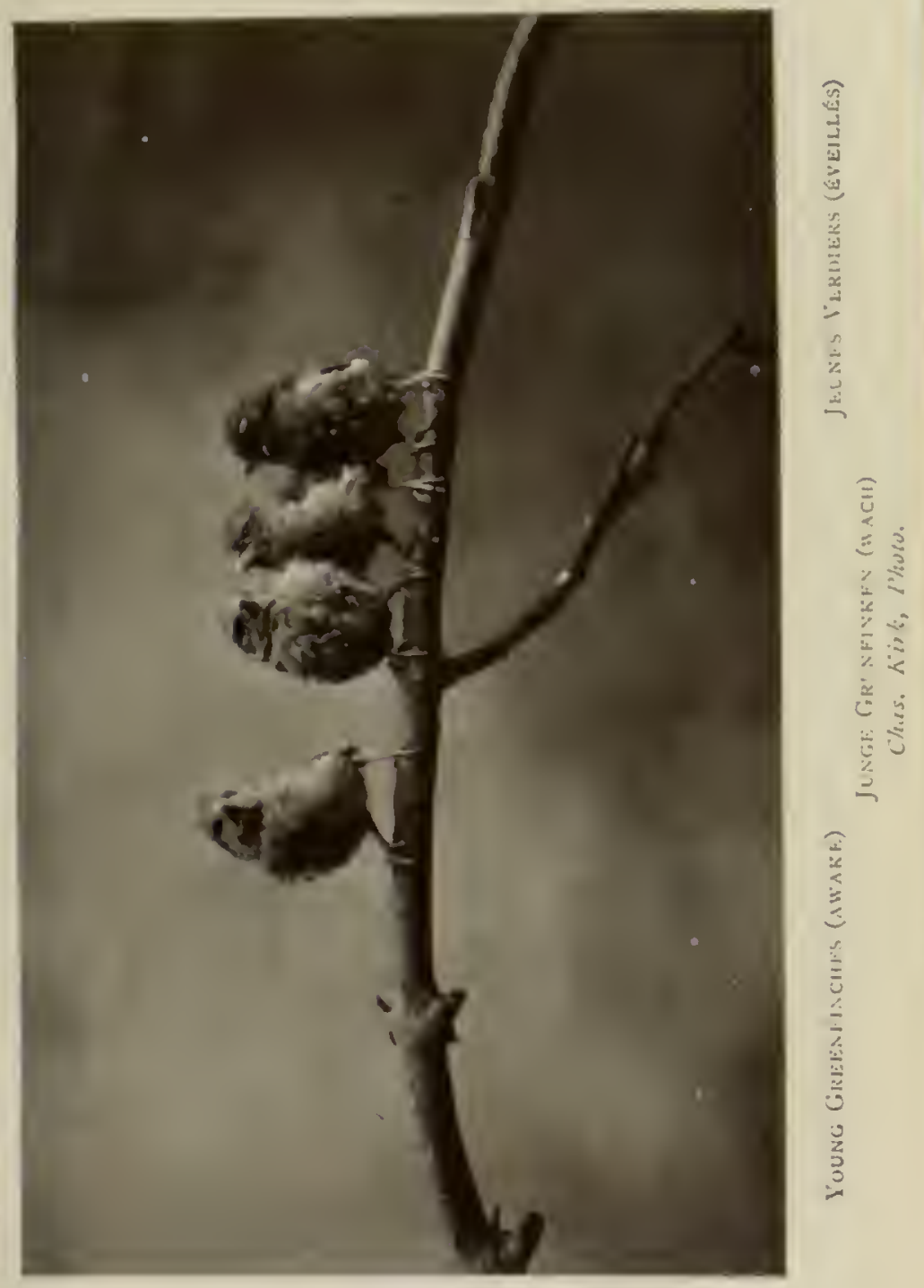




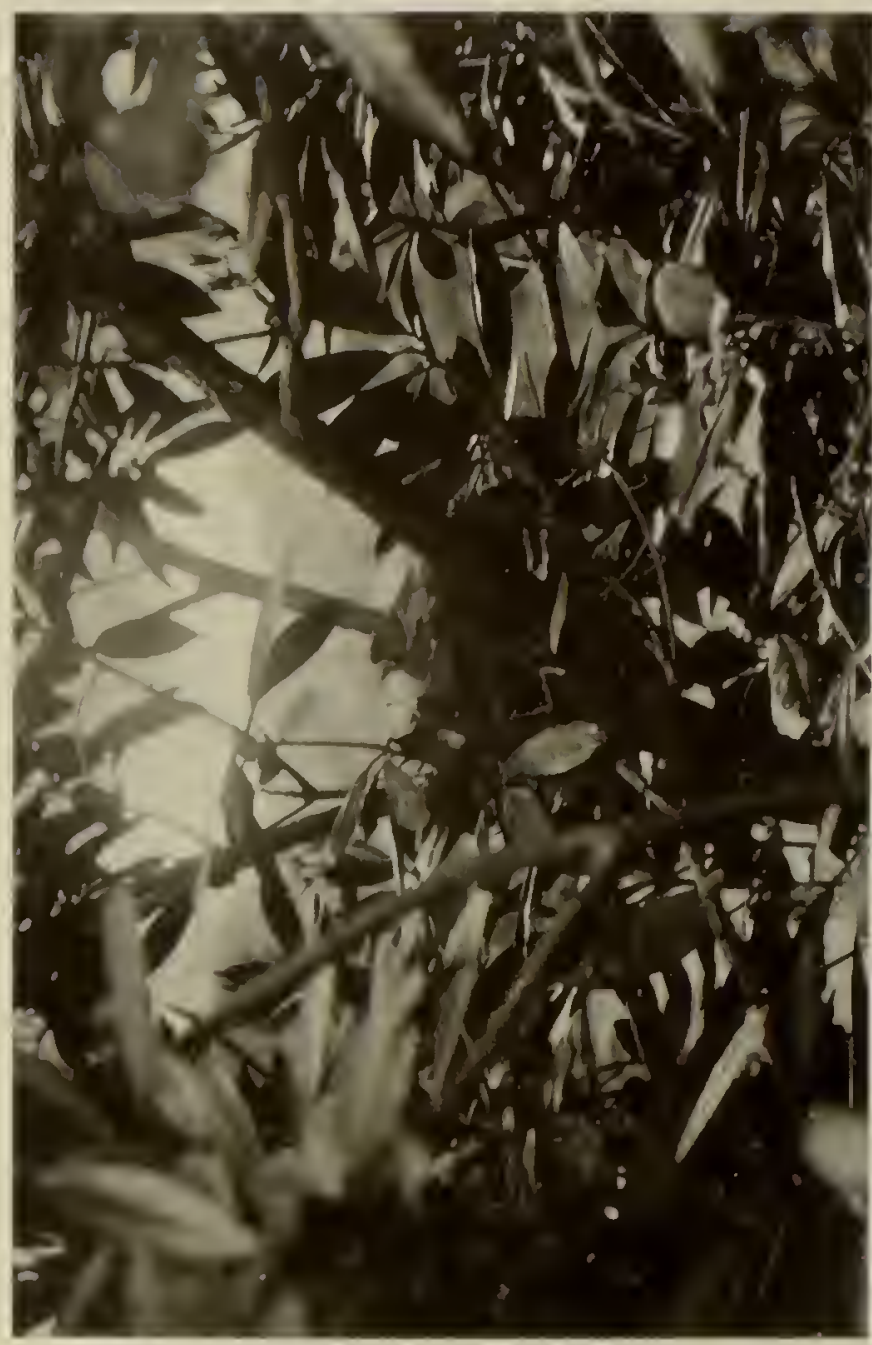

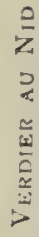

in
$z$
$z$
$z$
$z$
$z$
$z$
$z$
$z$
$z$ 


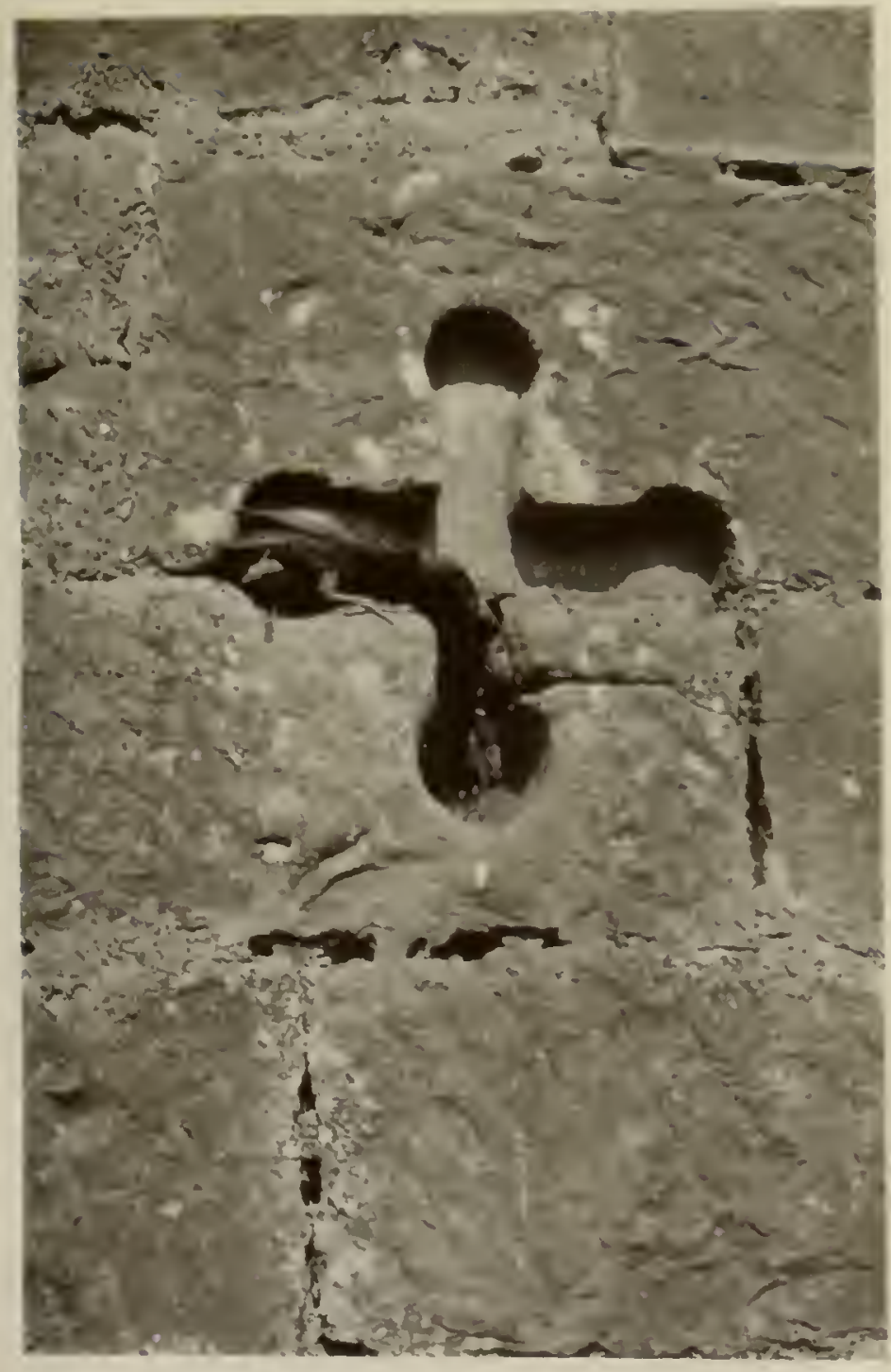

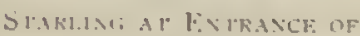
Nir.

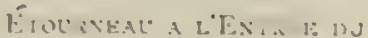

Vis

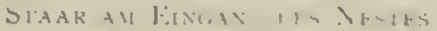




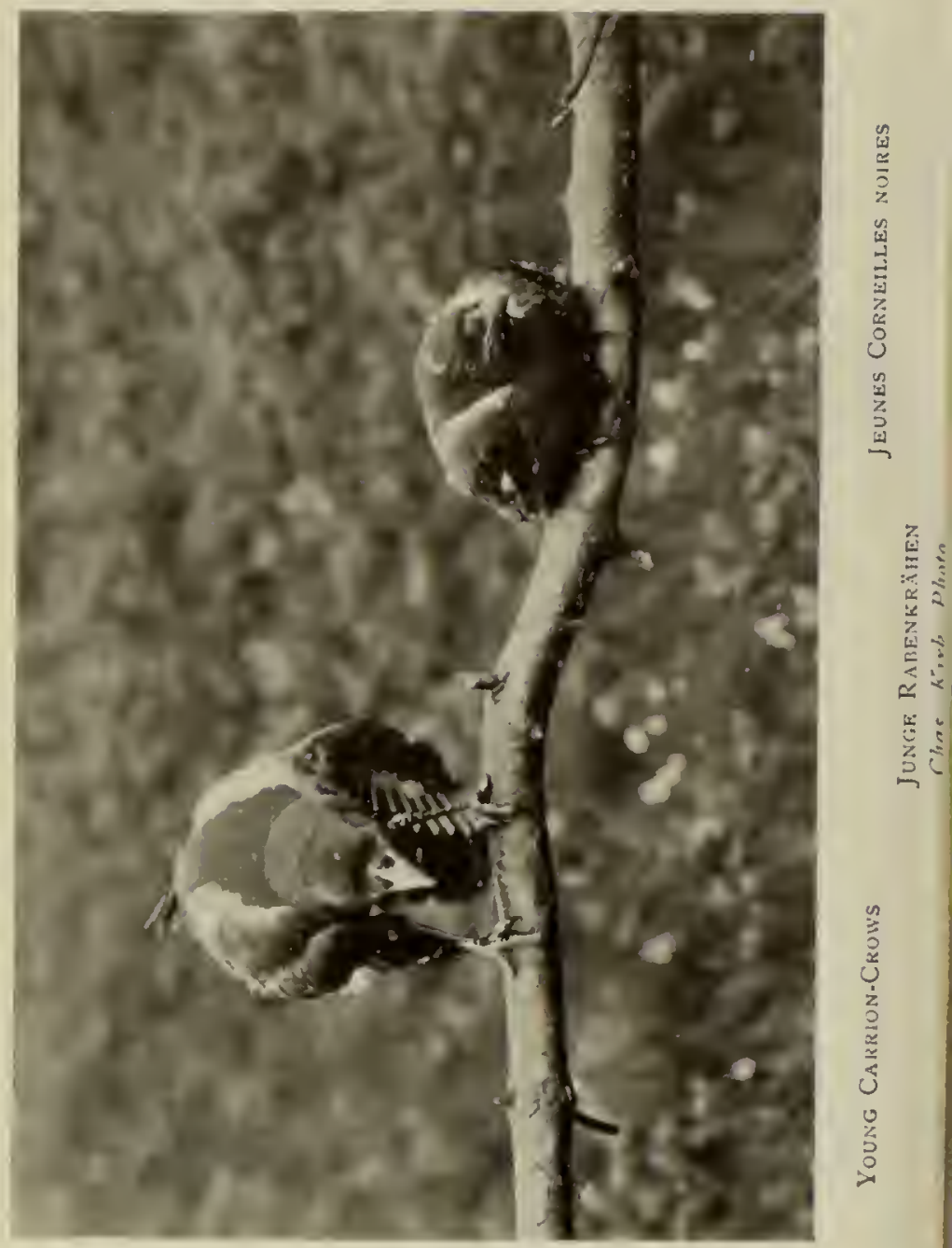


CORVUS CORONE, L.

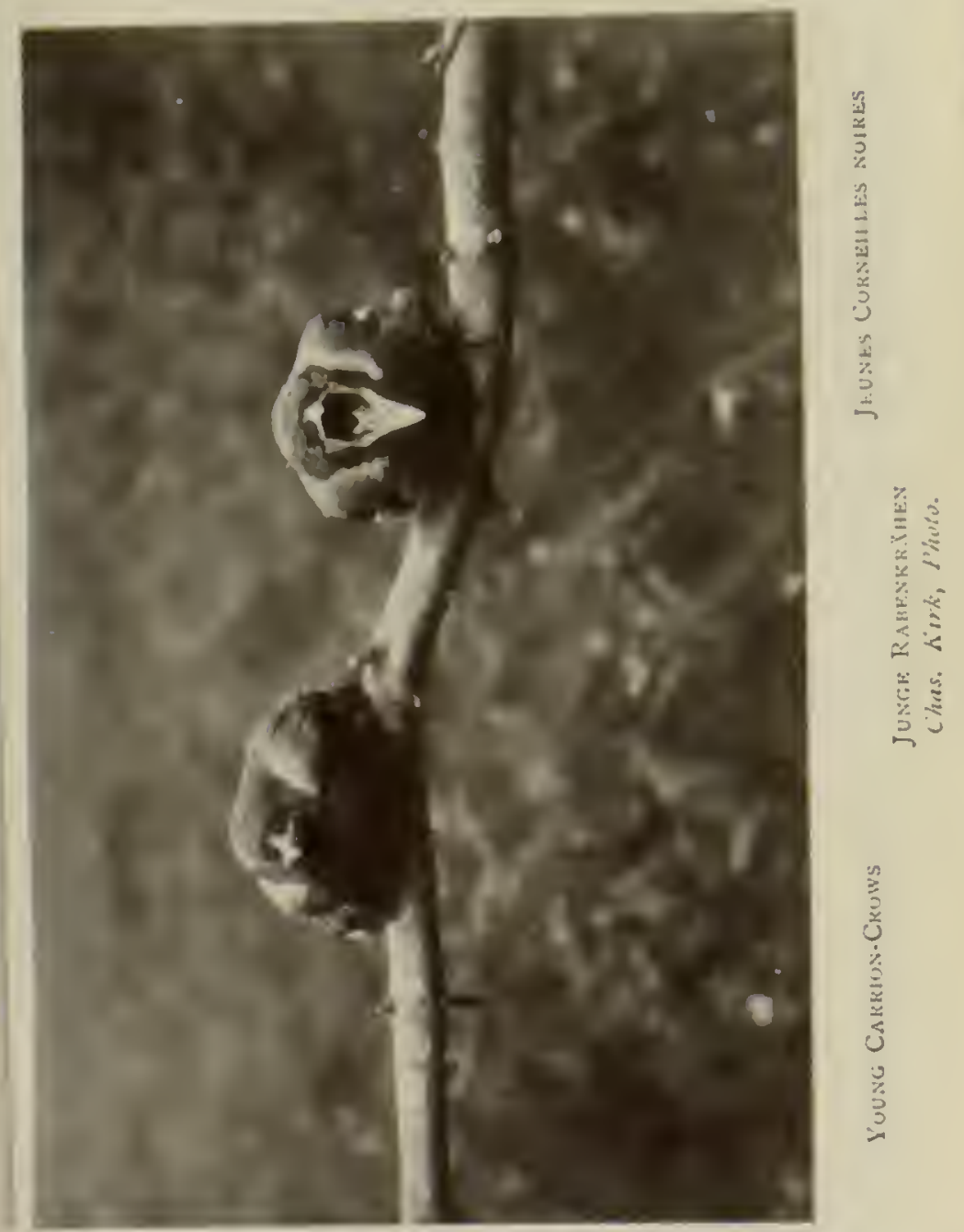




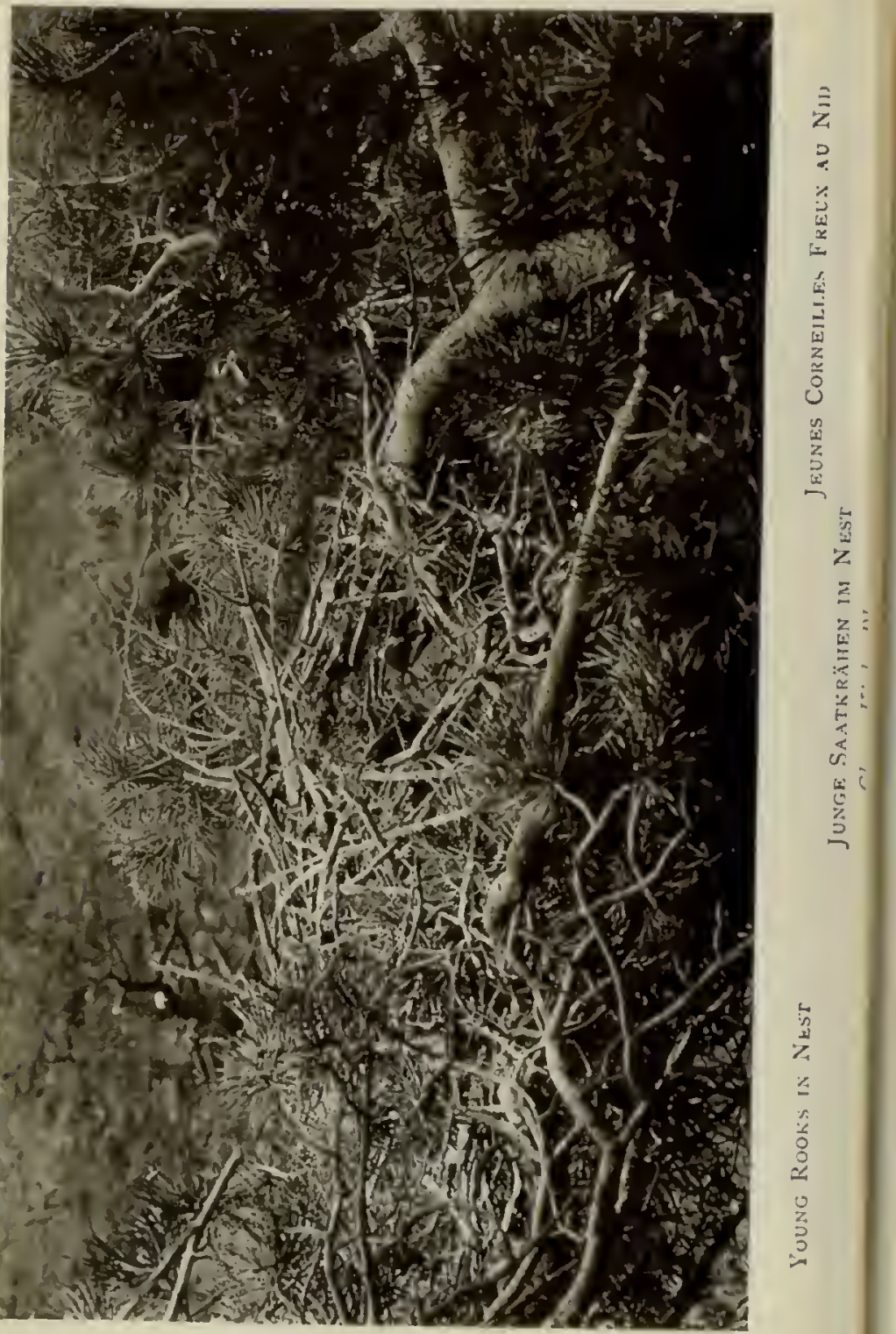




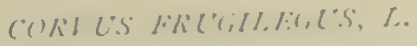

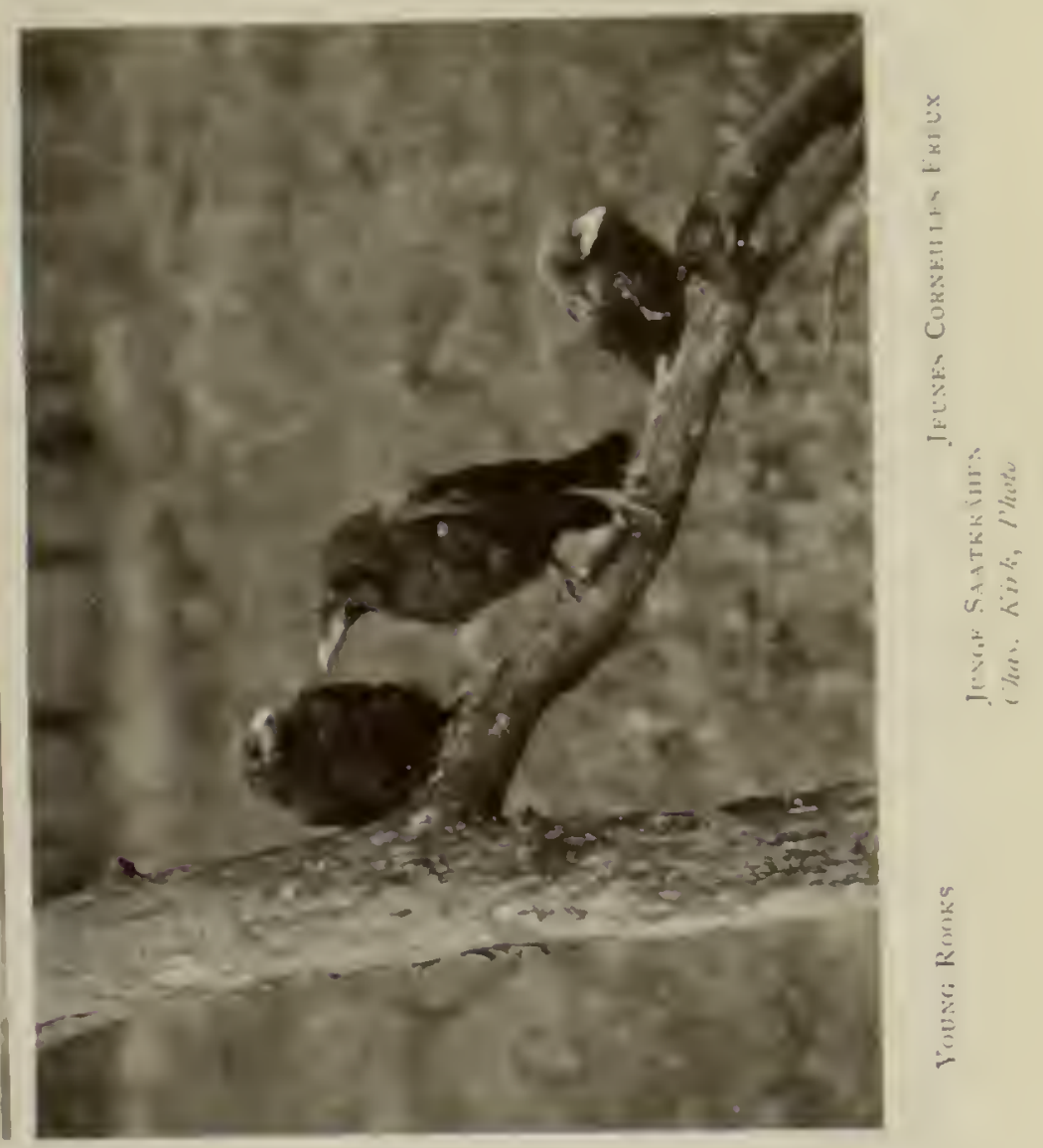




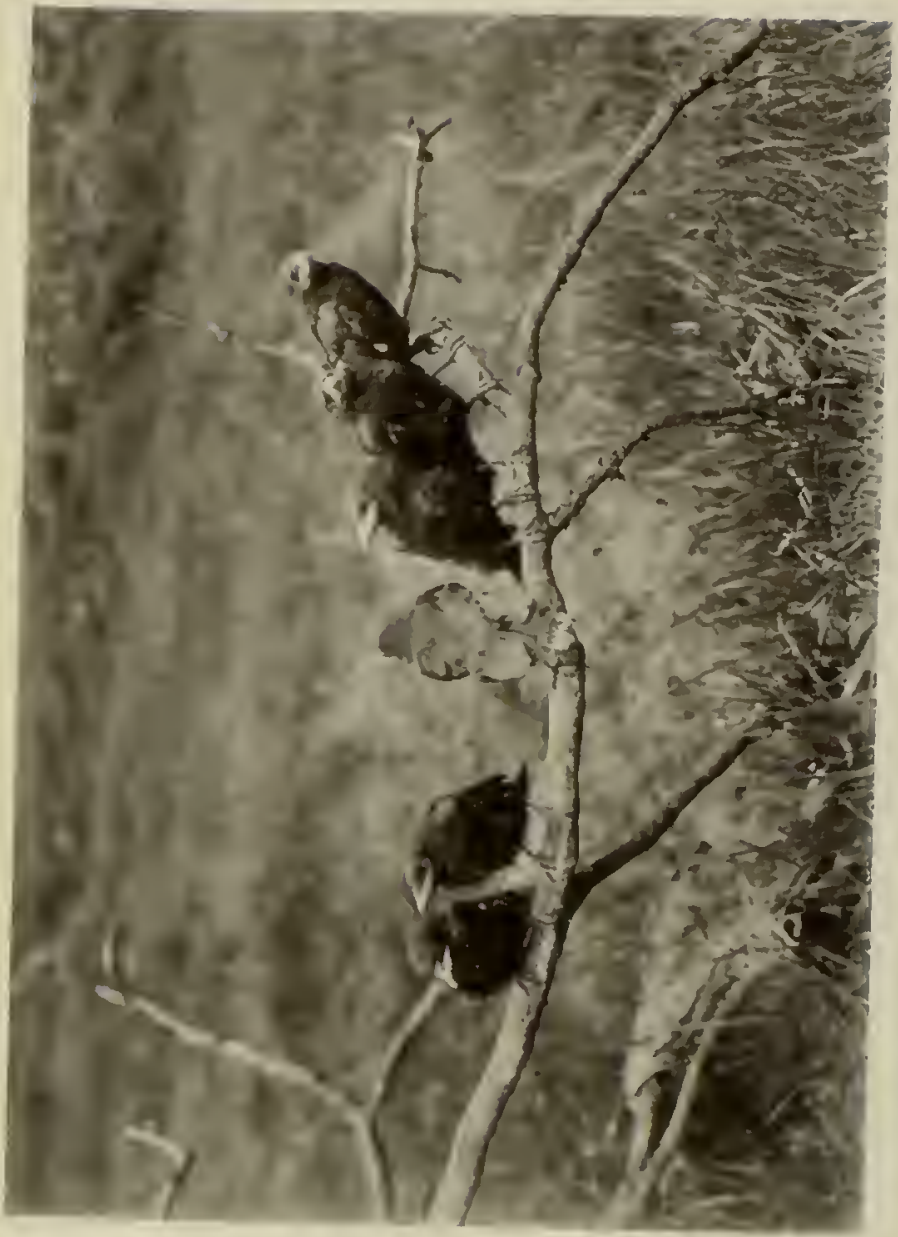

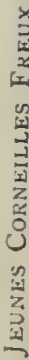

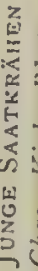

4
1
0
0
0
0
5
0
0 


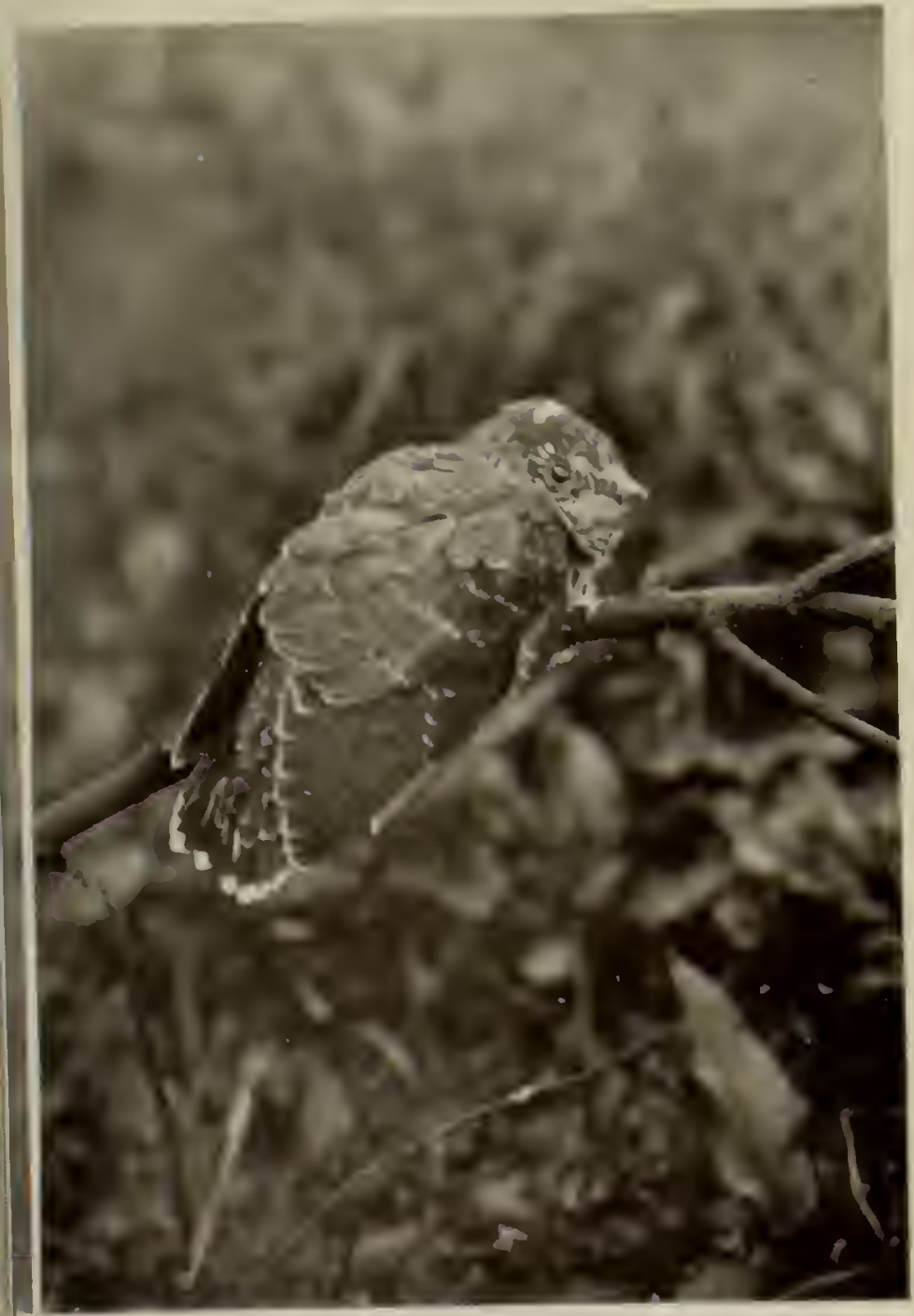

Yousc Cuchoo 


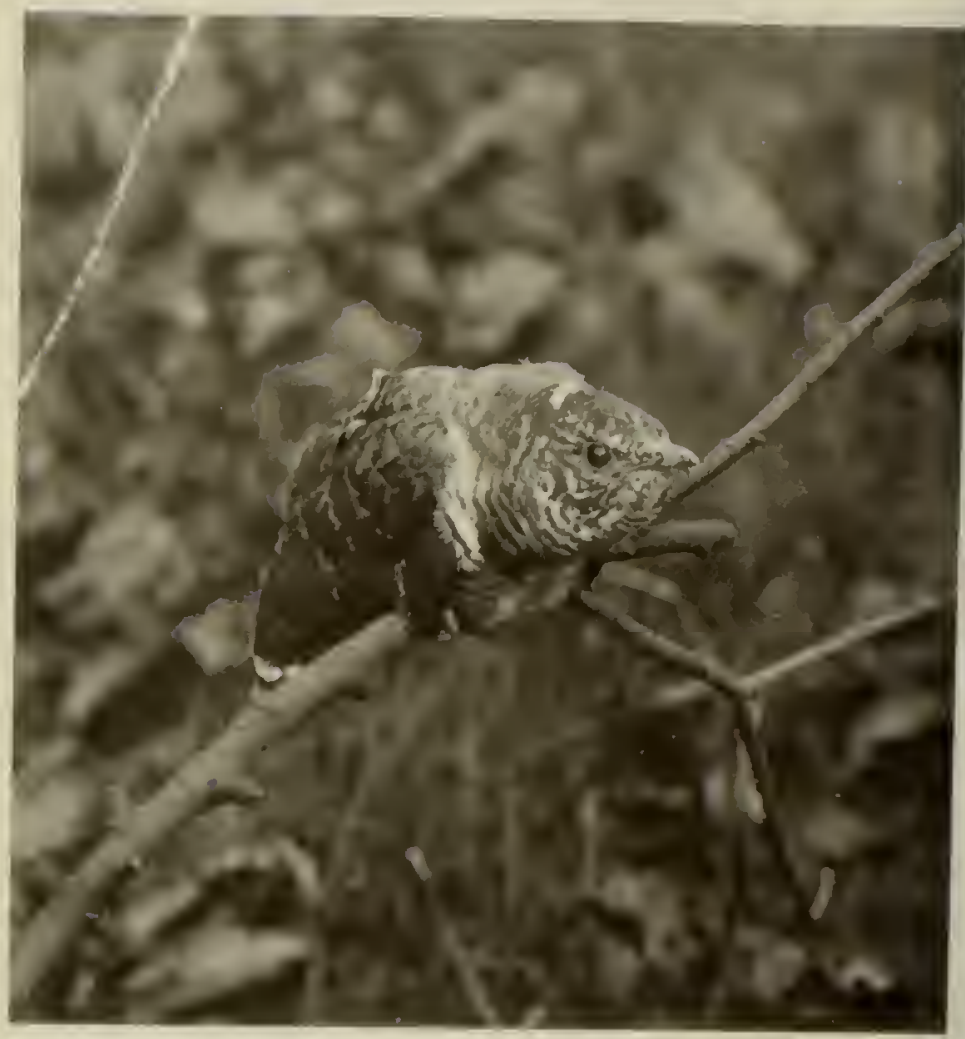

loung; Cuckou

Juverke Luckuck

Chas. Ni\%, photo. 


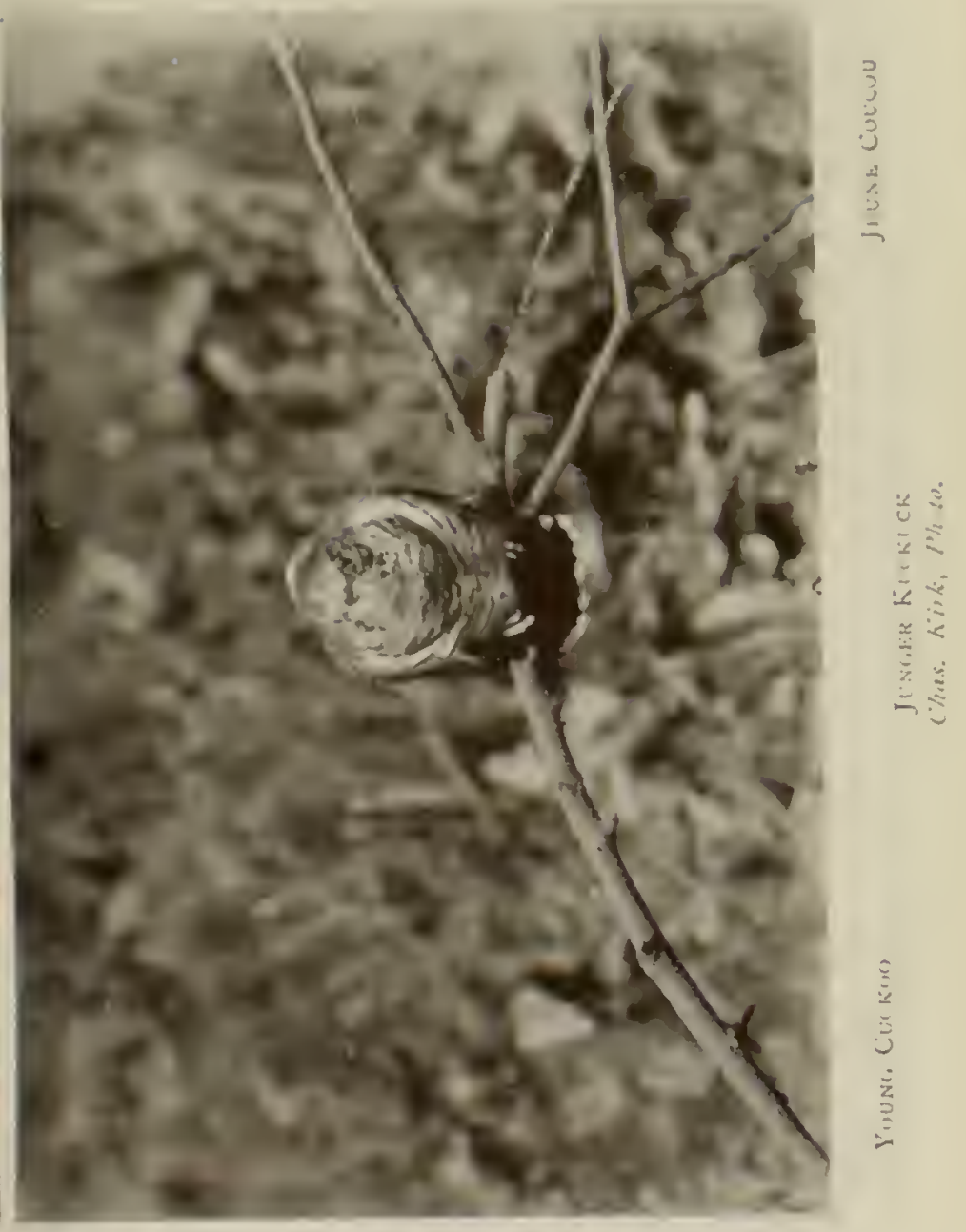




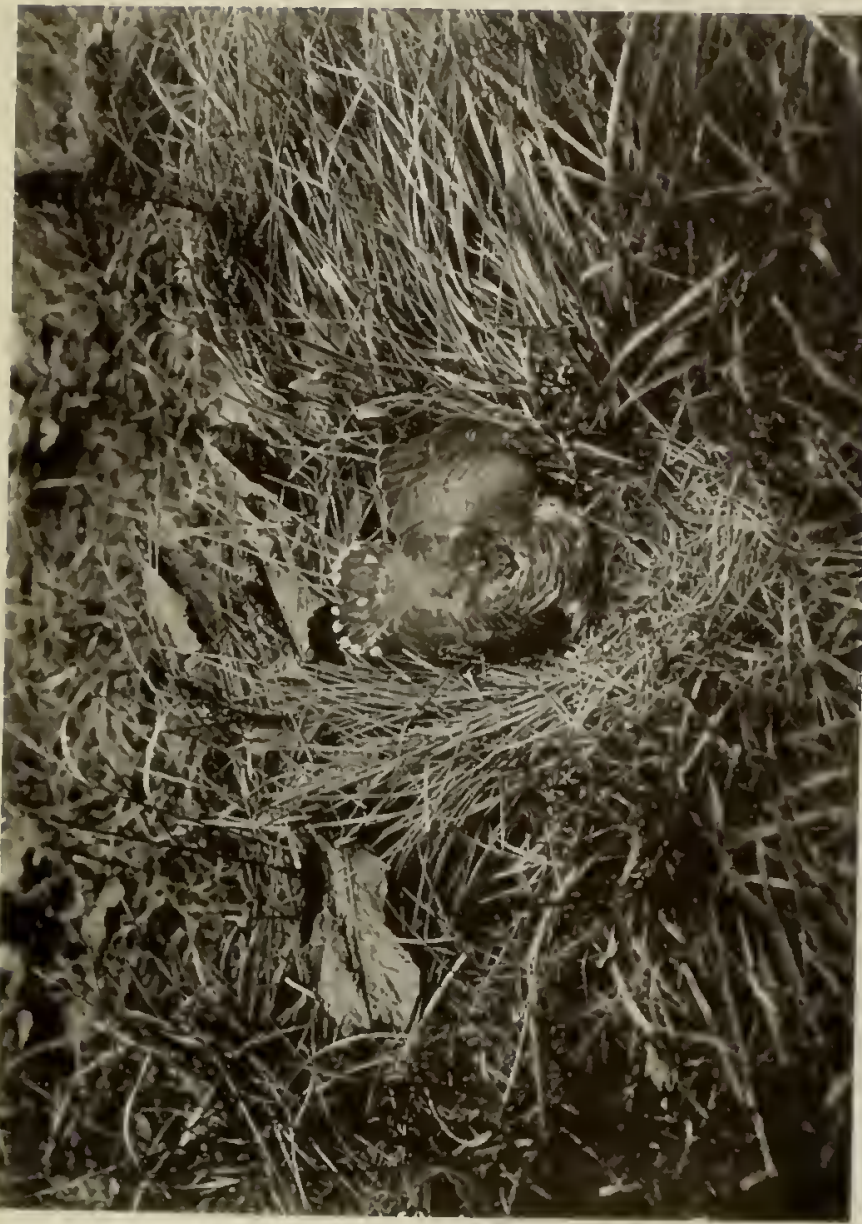

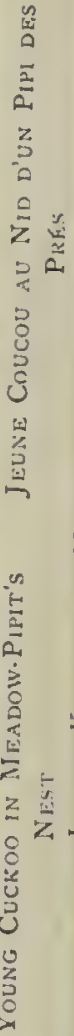




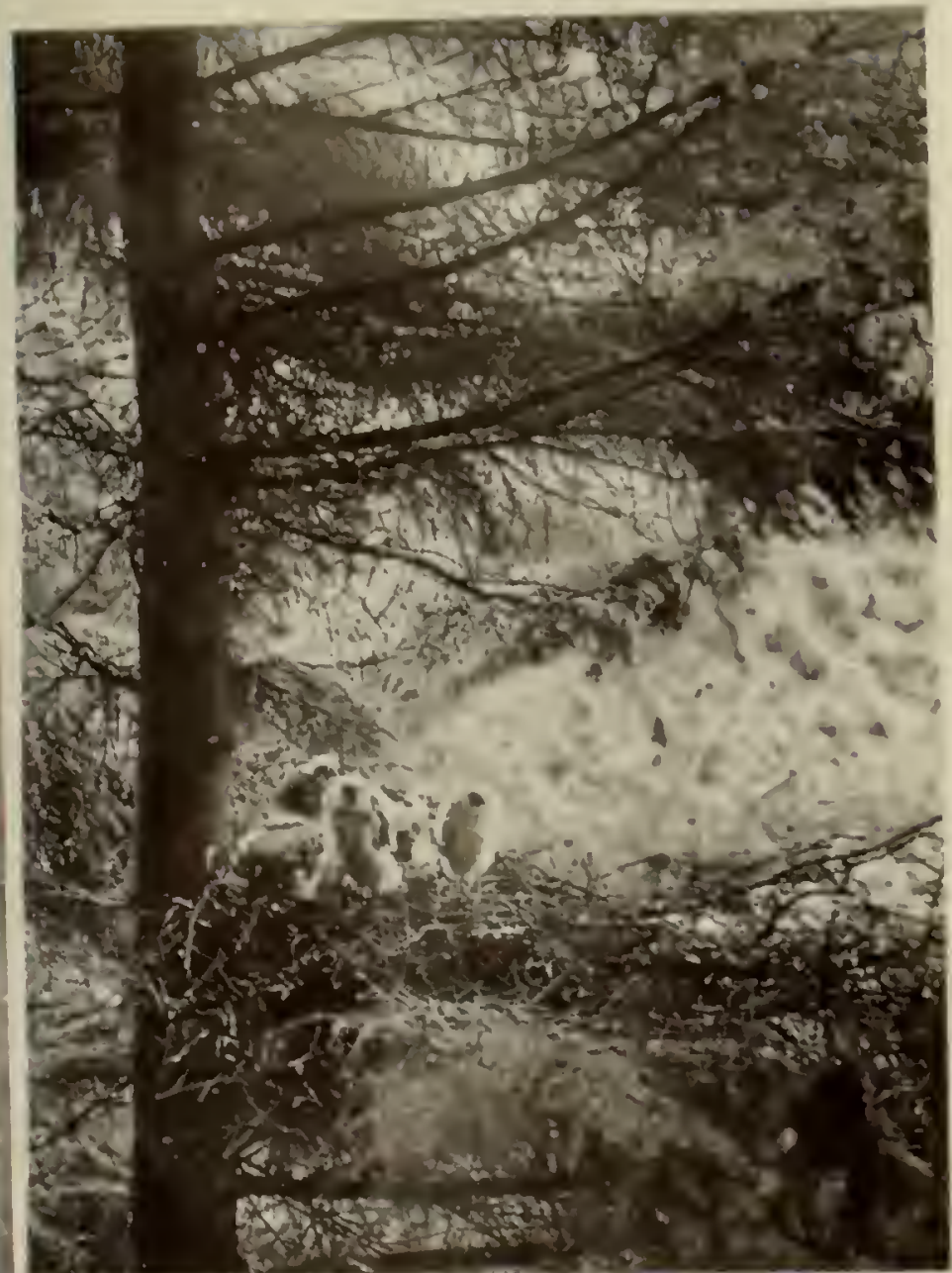

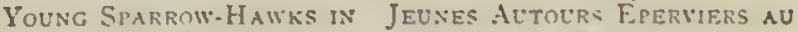
NEST Niv

JUNGE SPERBER IM SEST

Chas. Kirk, riw:o. 


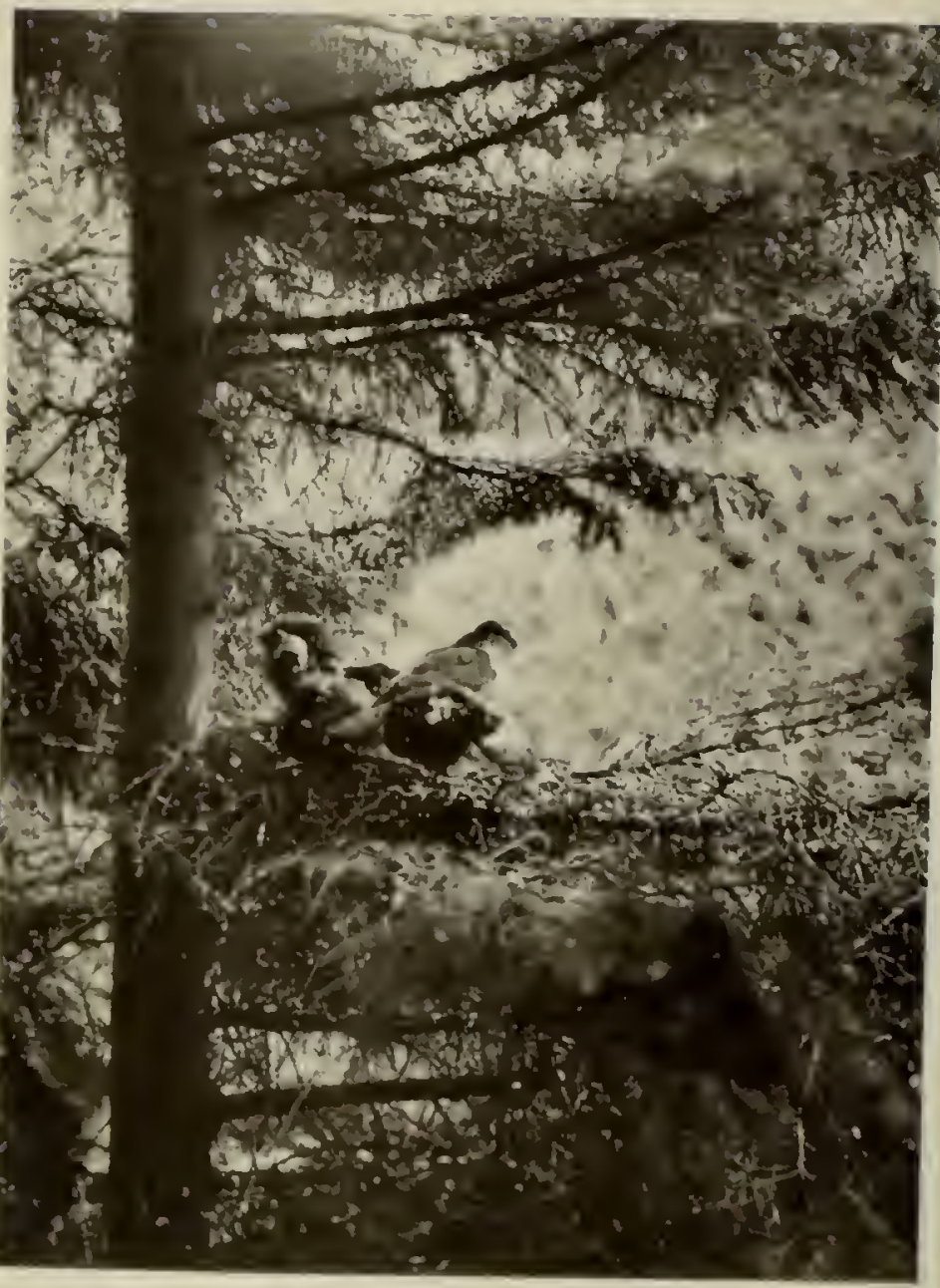

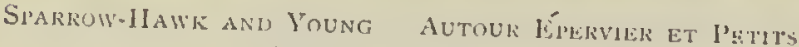
SHEKBER UND JUNGE

Clars. Airk, Photo. 


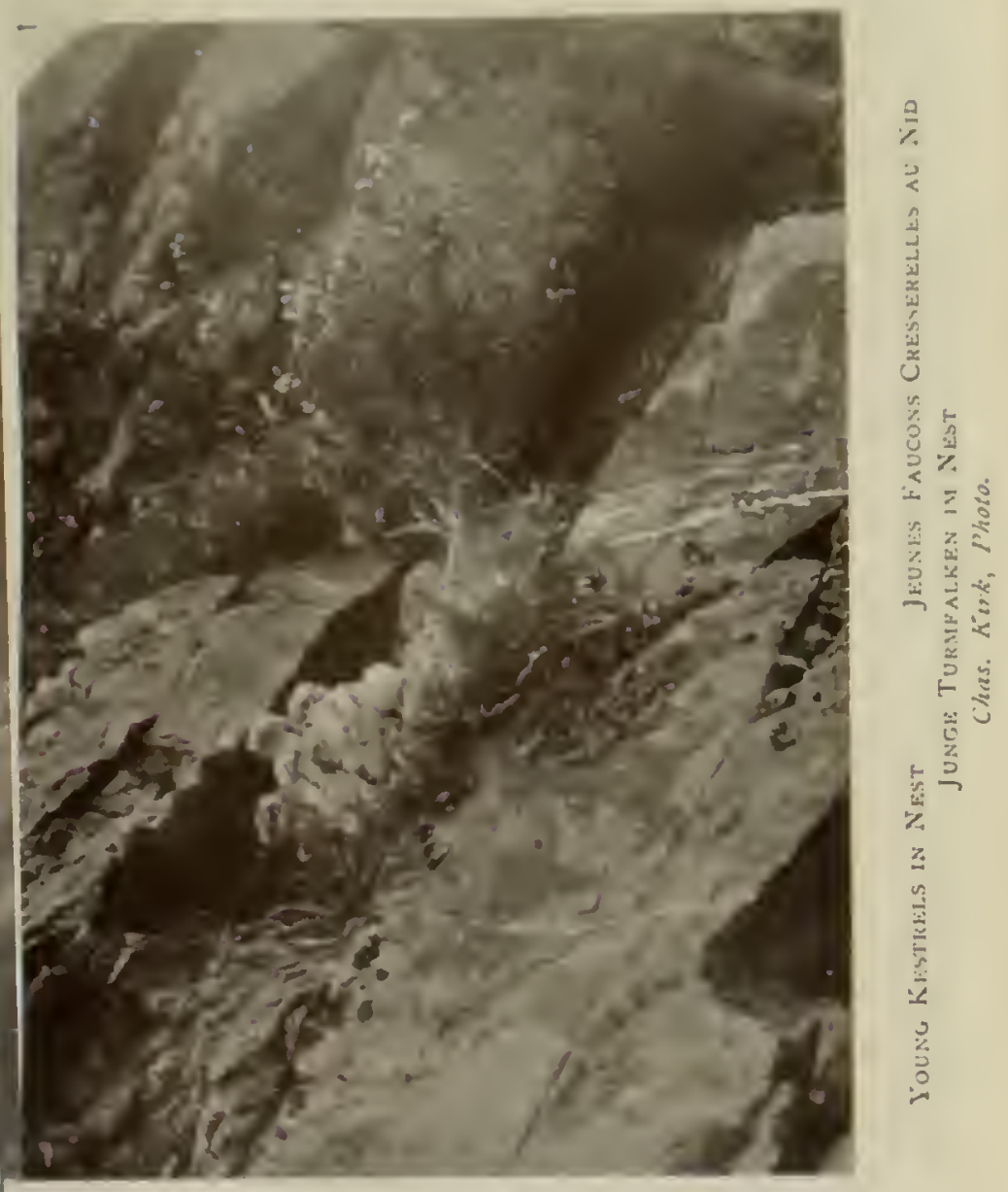


PHATACROCORAX' CARBO, L.

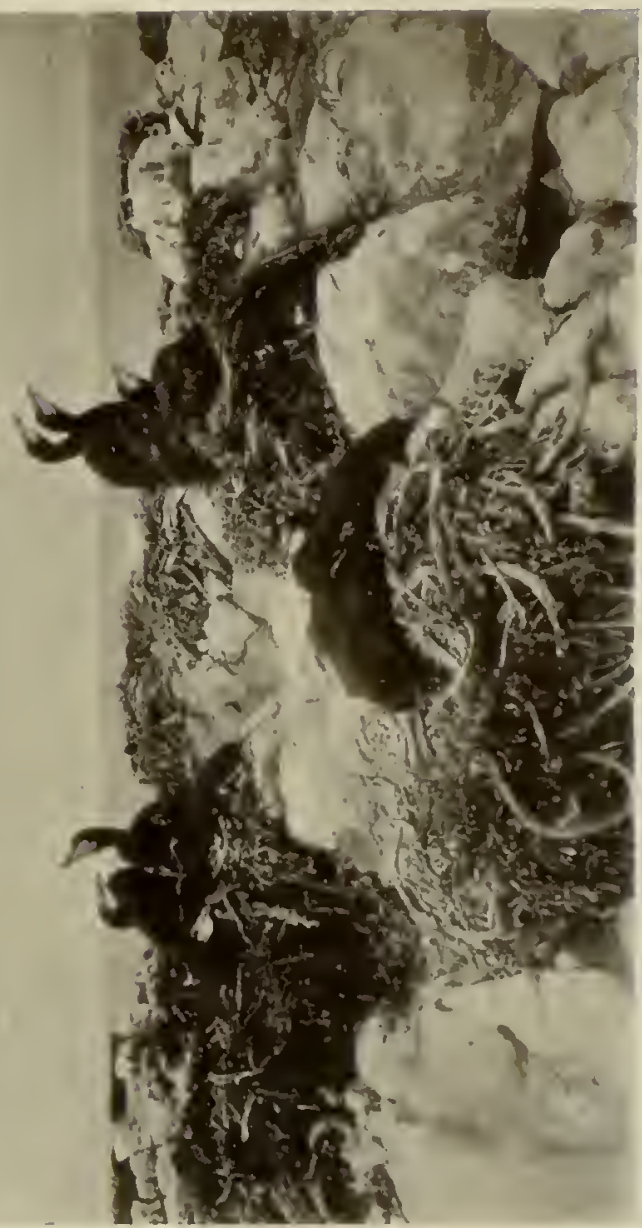

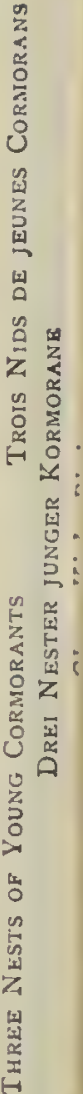




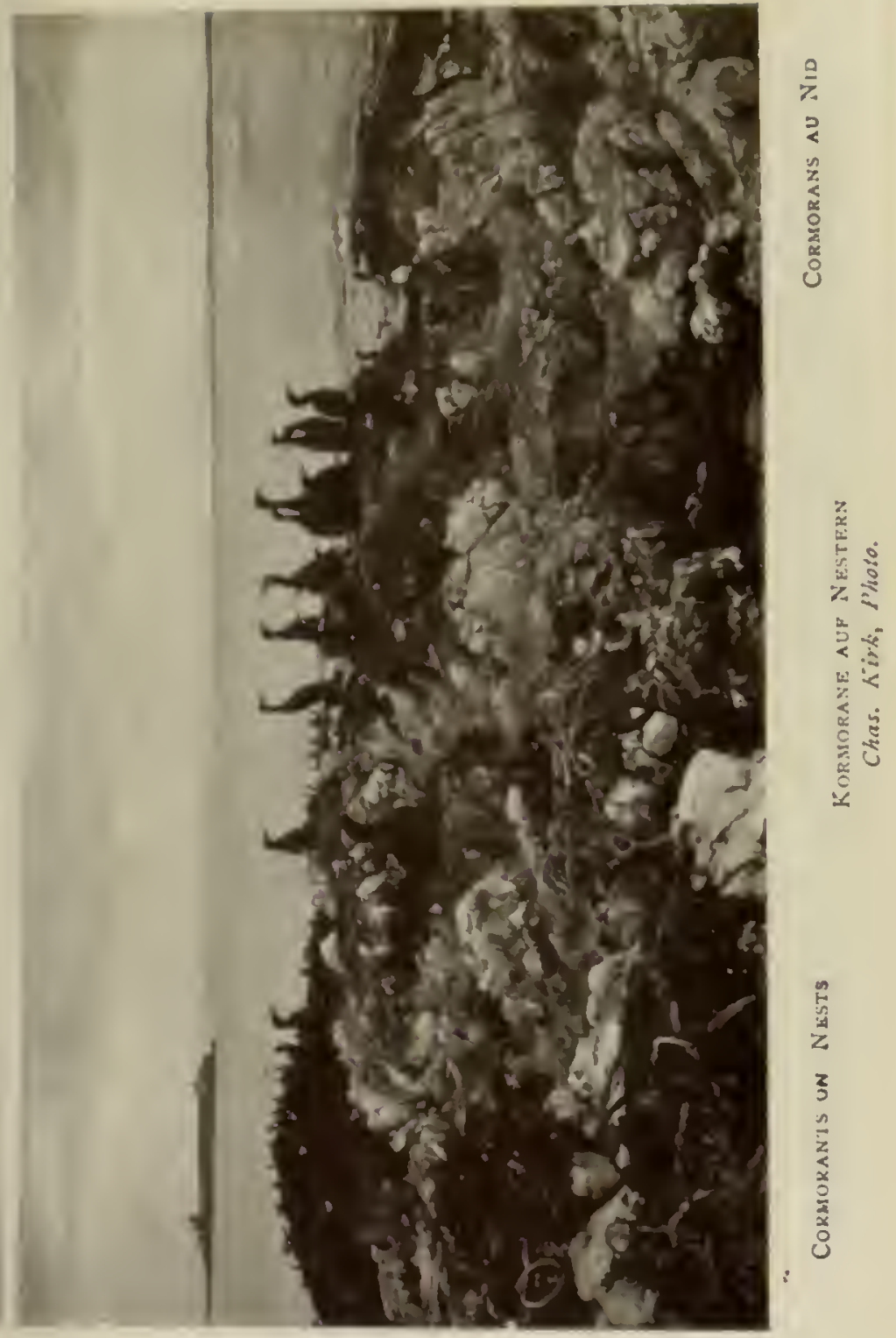




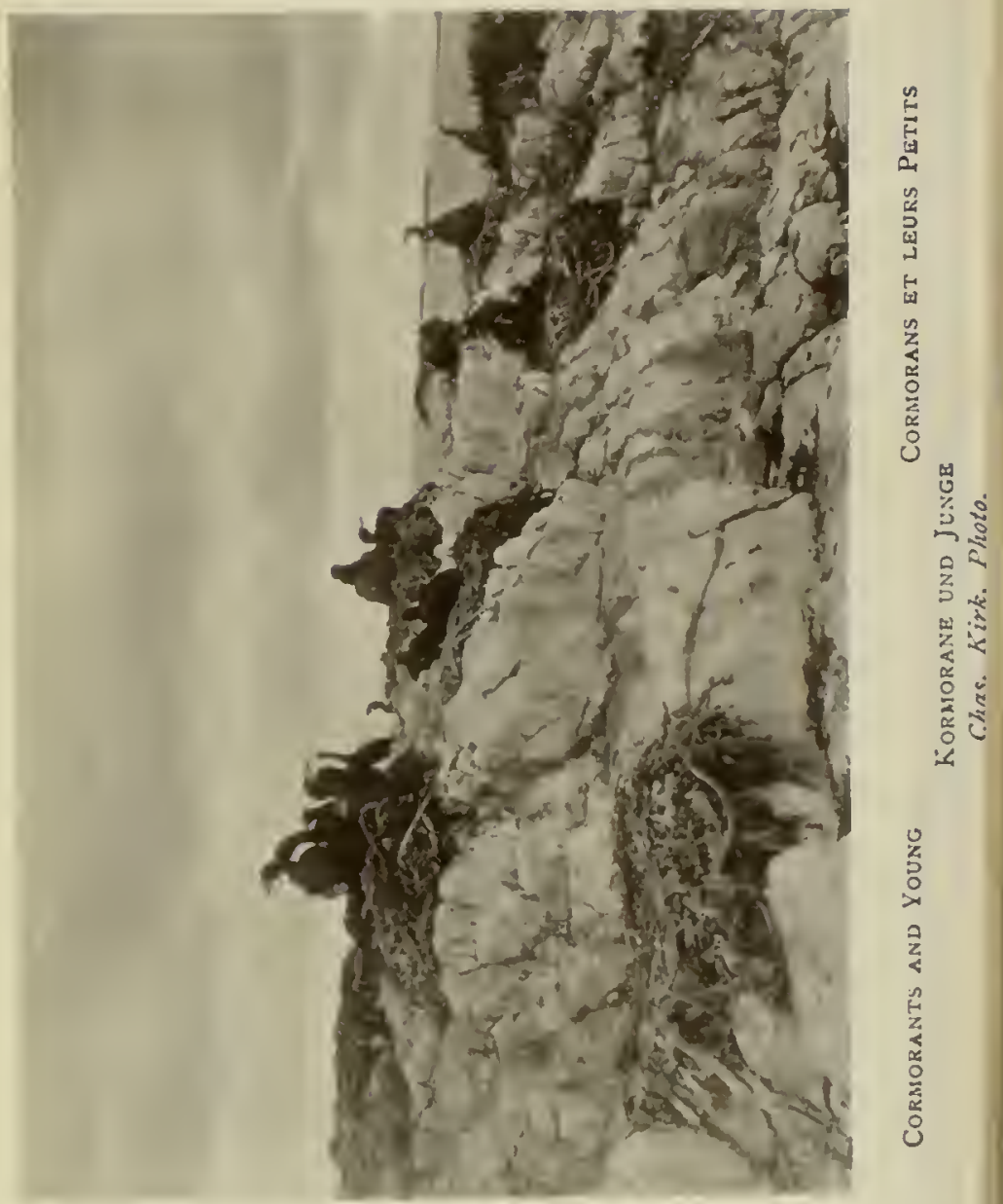




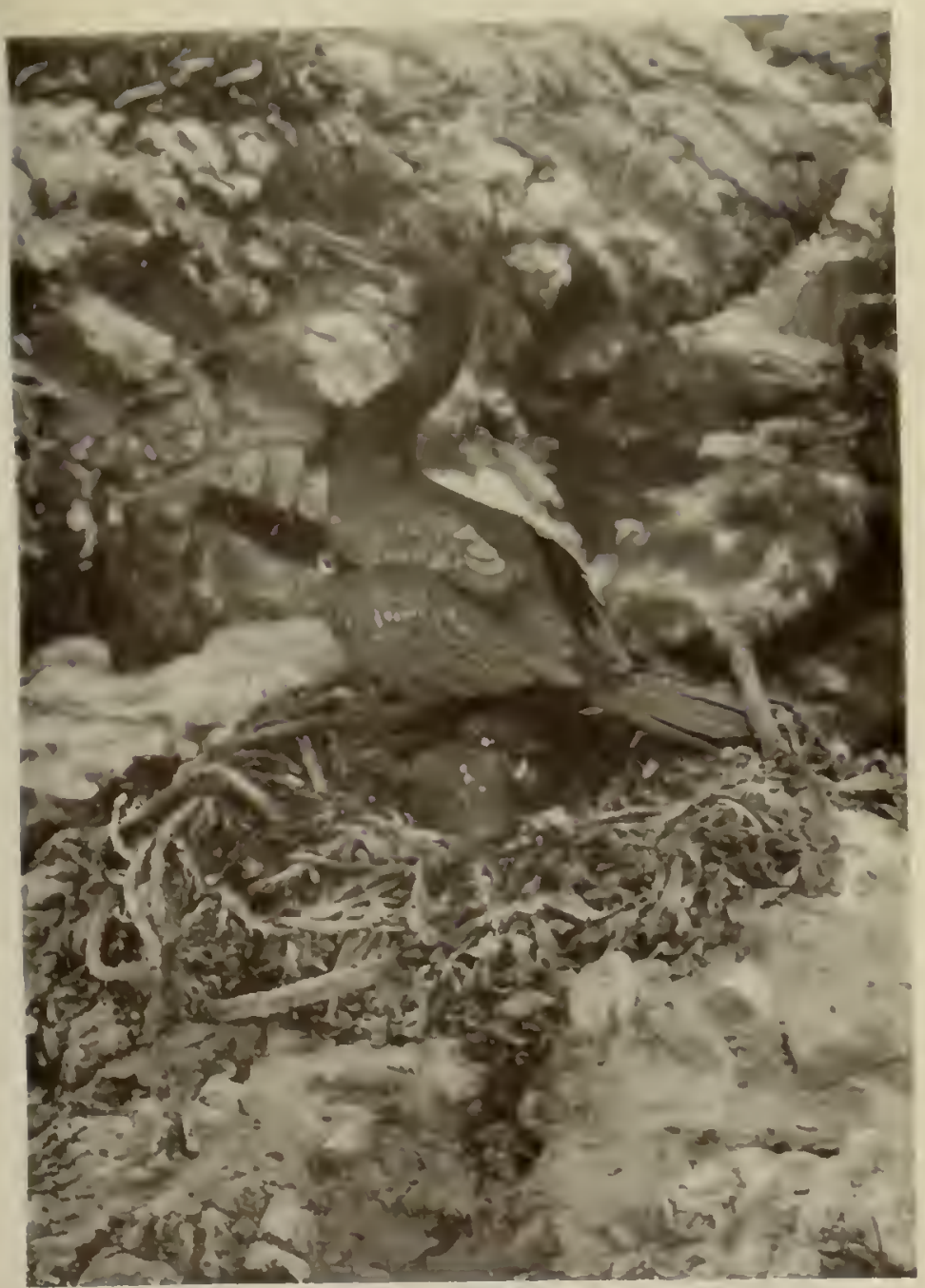

CukM Rant w Nest mTH loivg
Cormoras al Mido ateE SEJ

IETITS

KURMORAY IM NEST MIT JUNGEN

Chas. Nirk, Ficro. 


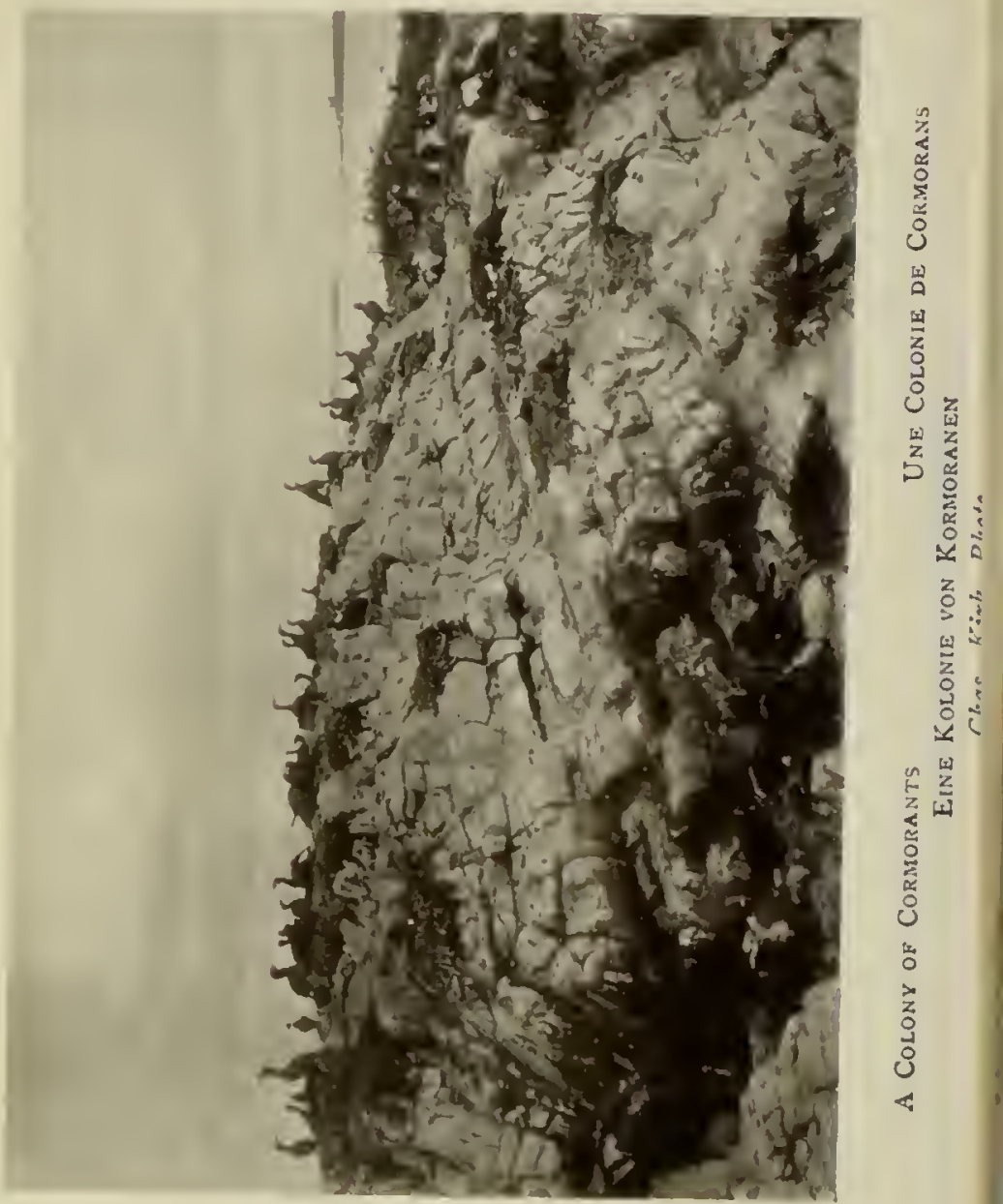




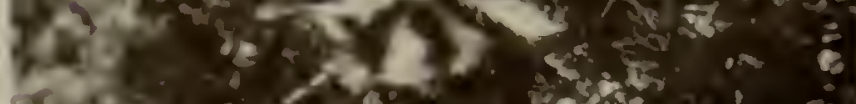

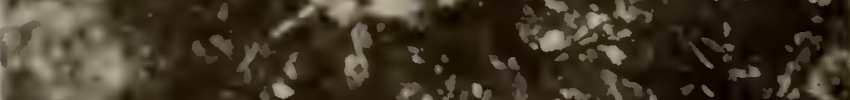

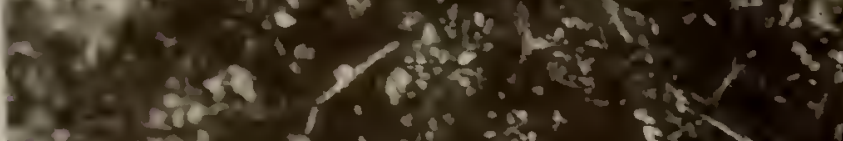

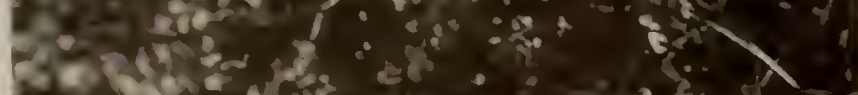

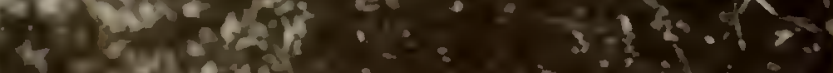

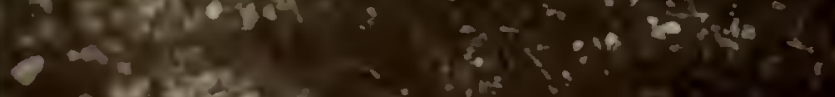

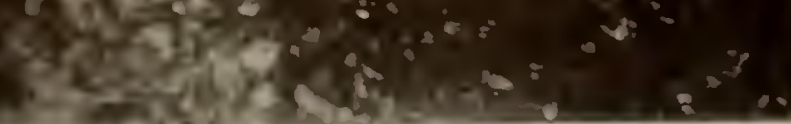




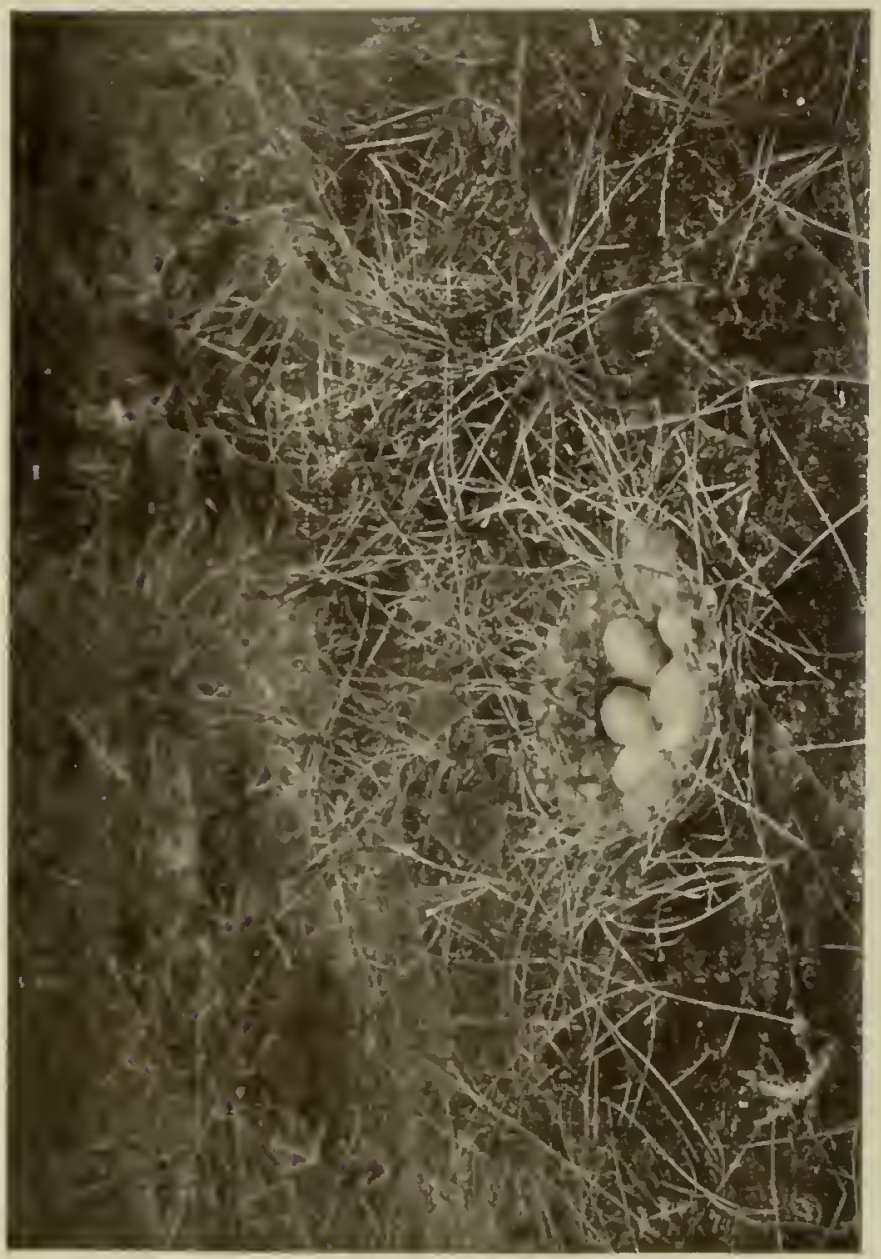

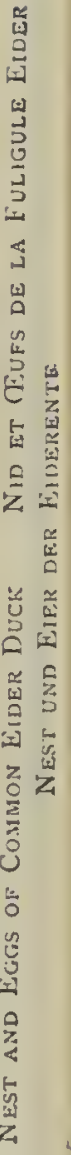




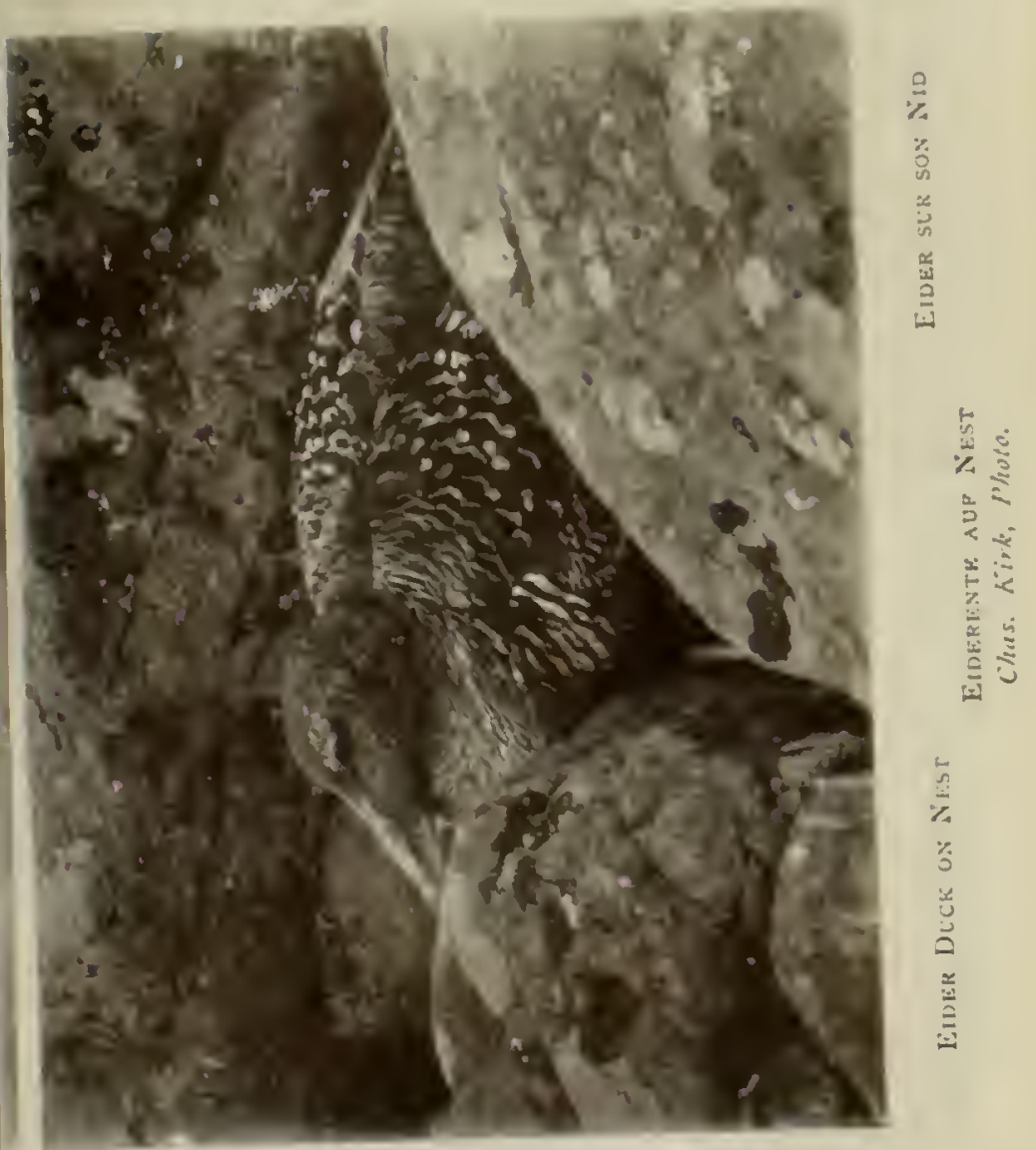




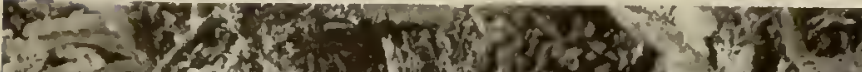

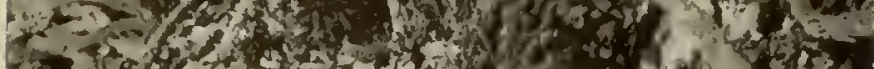

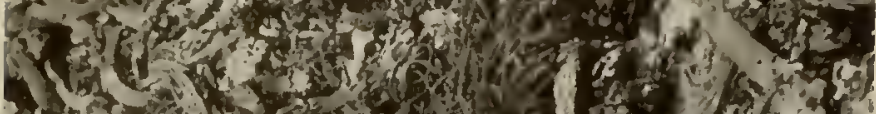

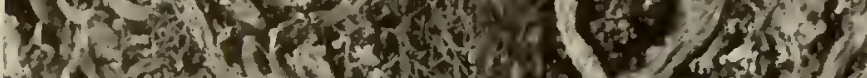

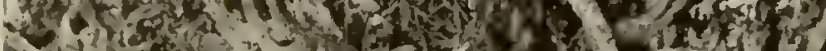

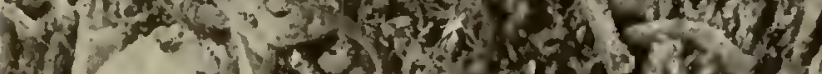

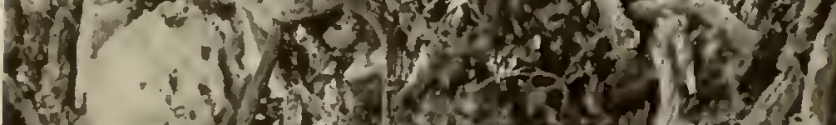

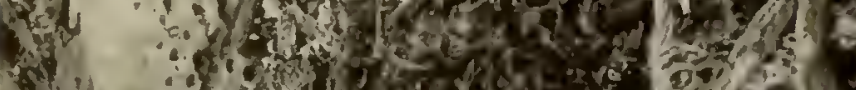

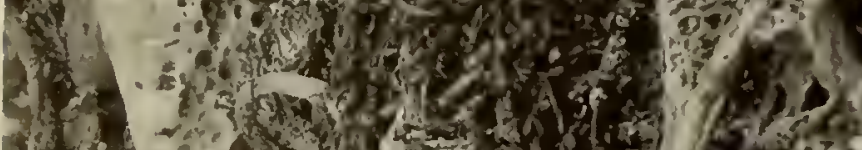

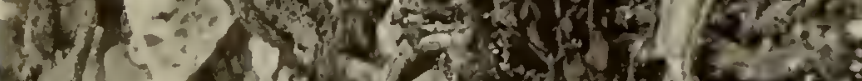

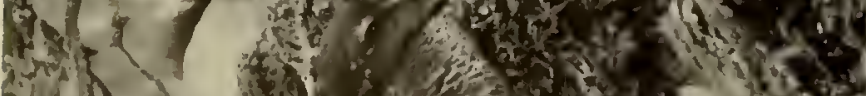

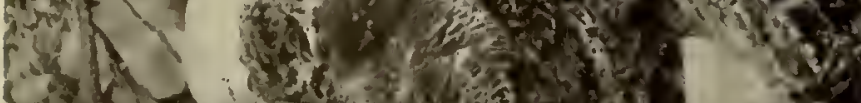

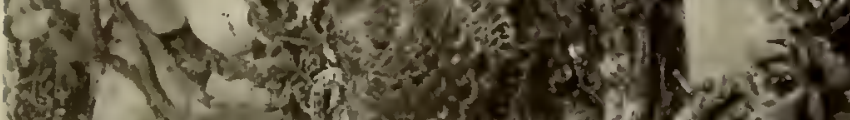

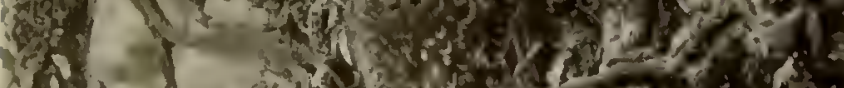

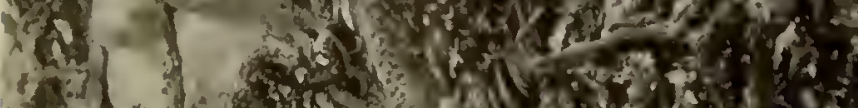

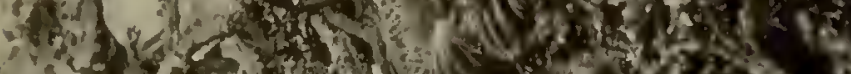

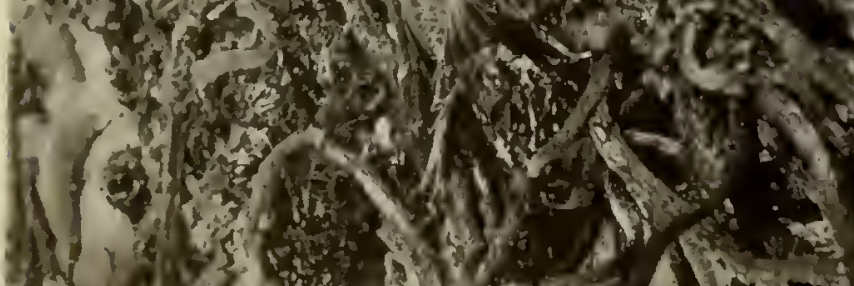

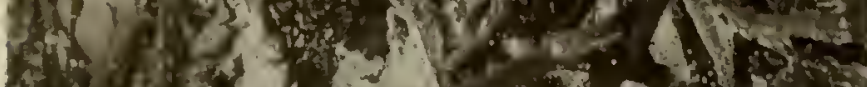

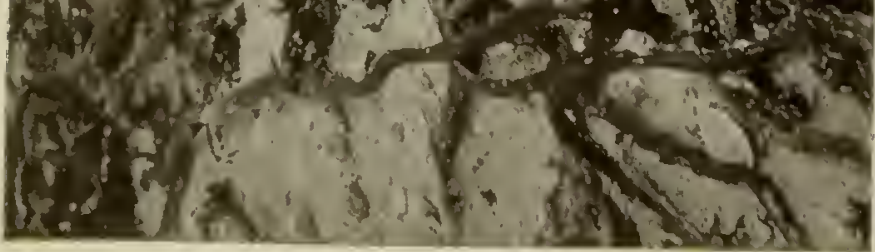




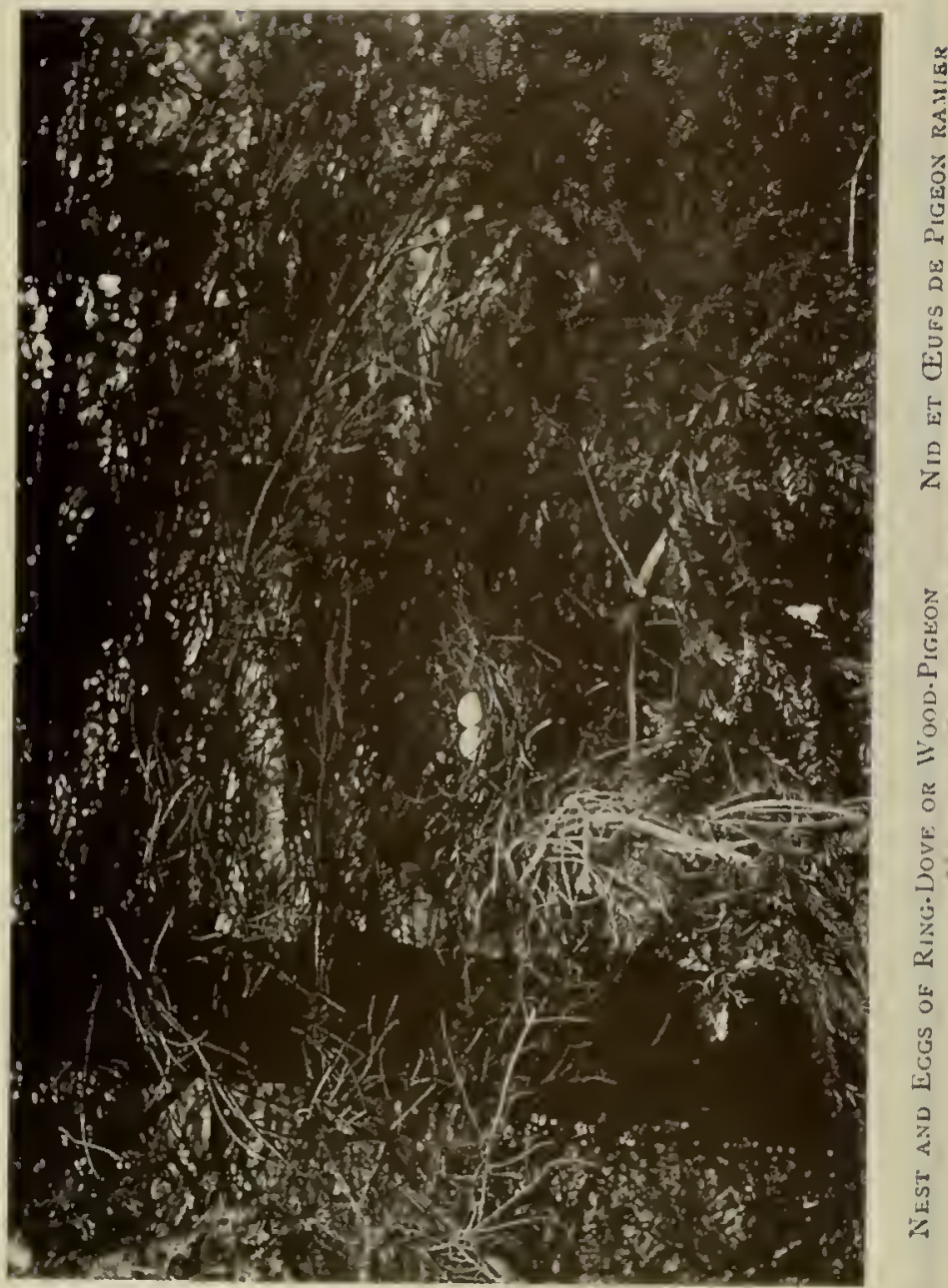




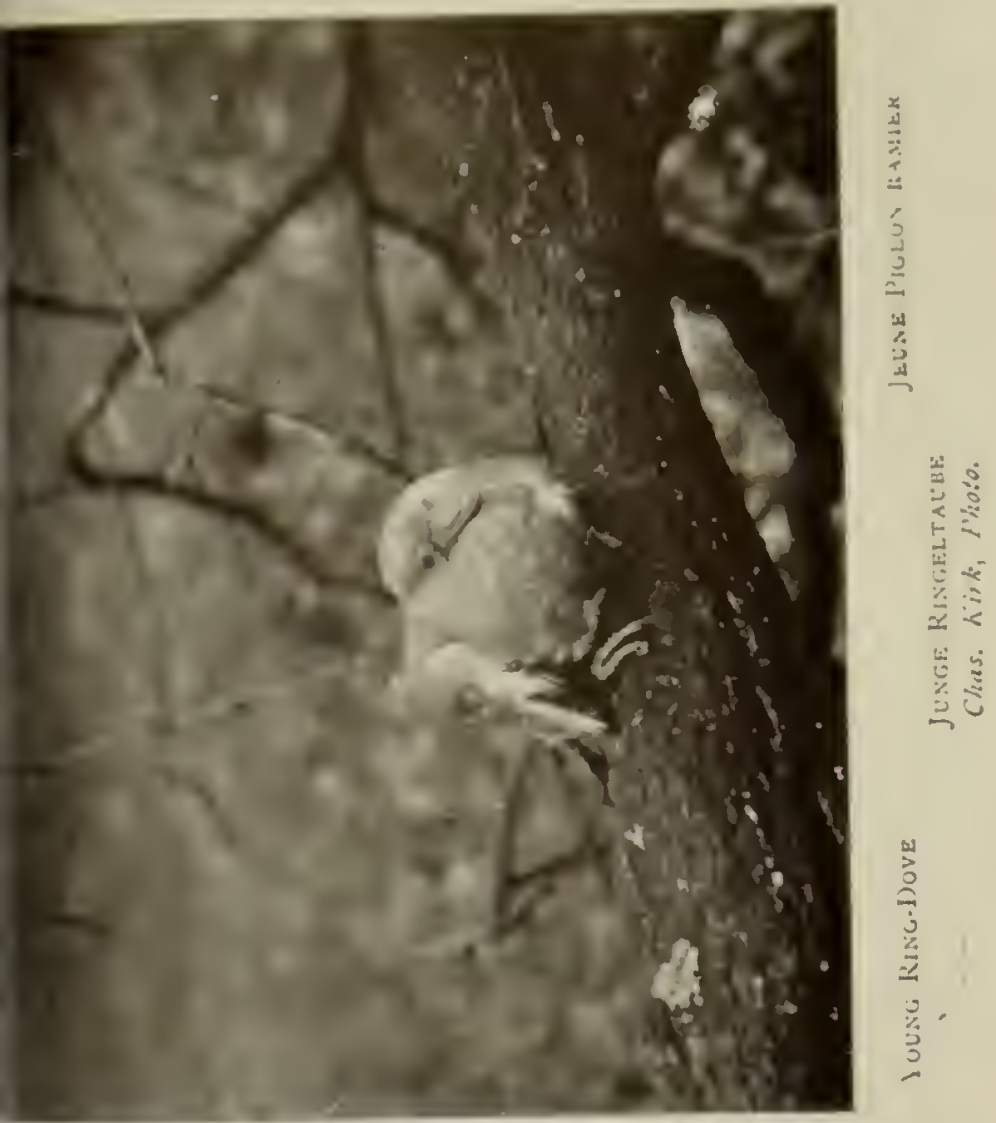




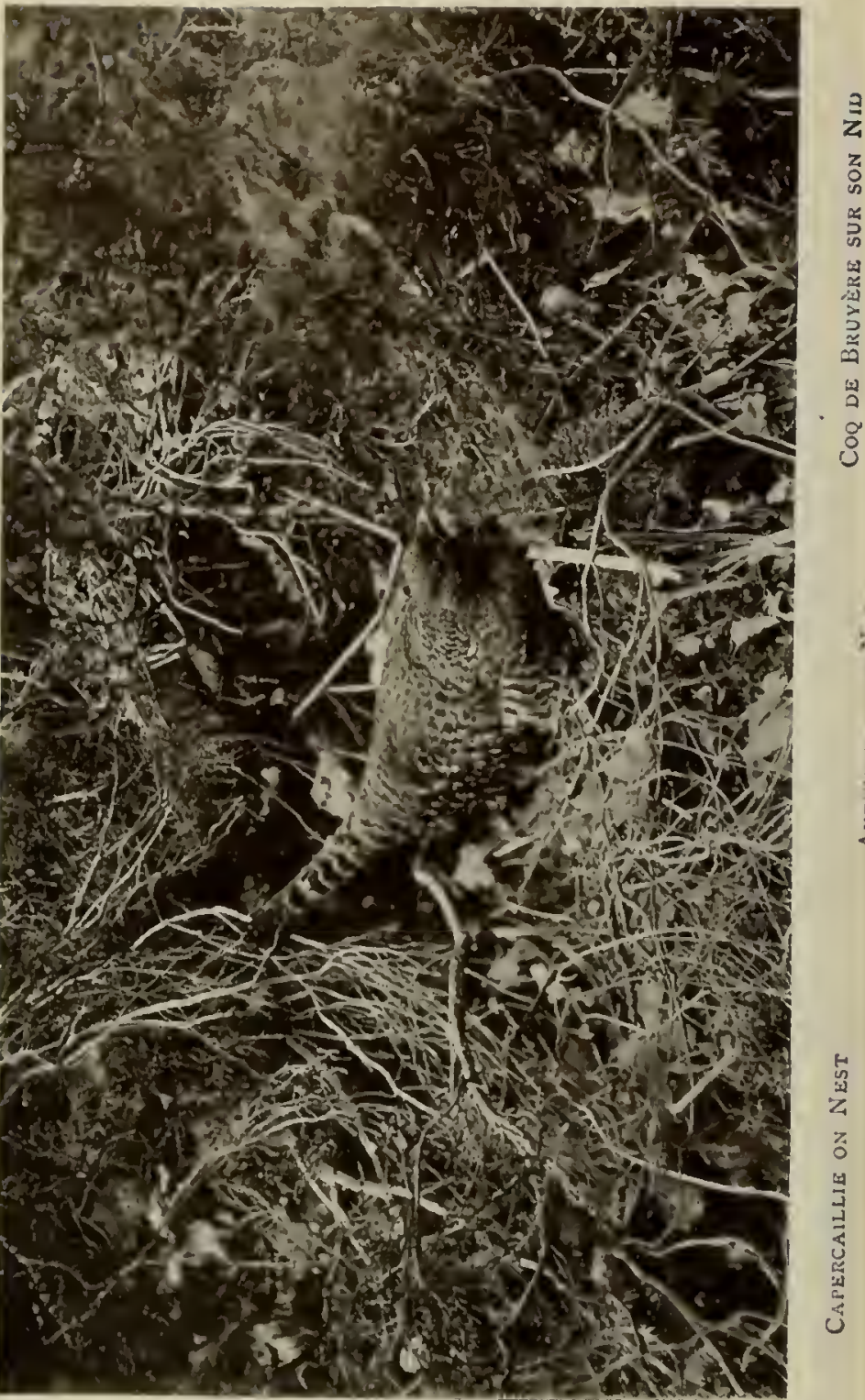




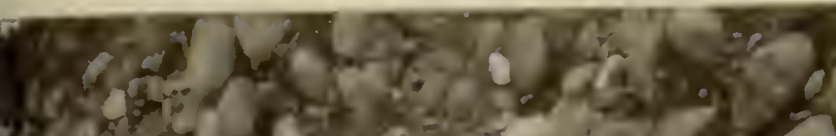

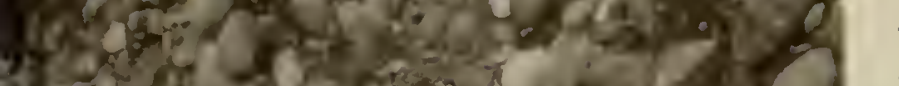

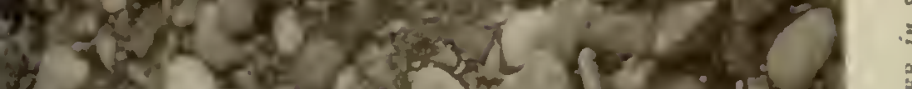

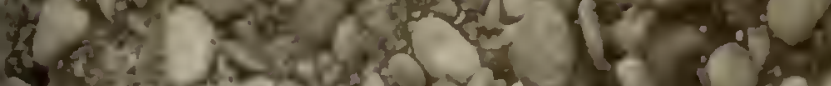
and

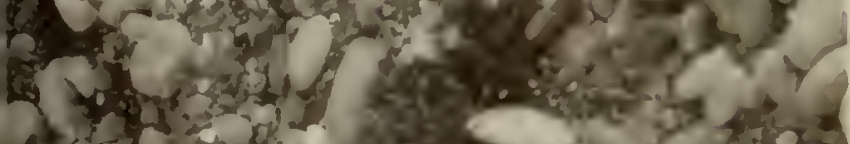

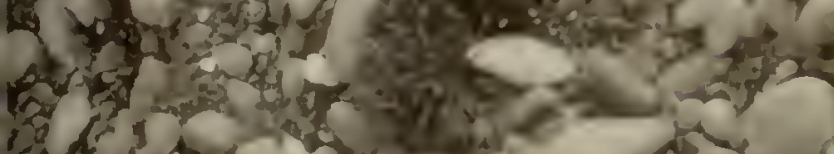
ind

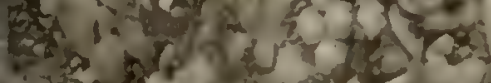

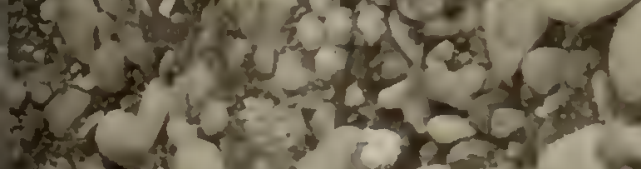
i. 20. $x+x-2 x$

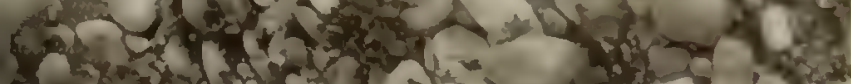

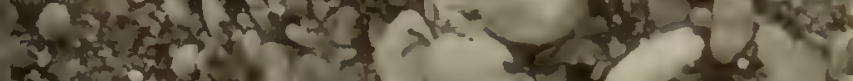

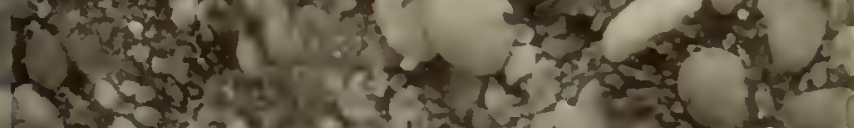

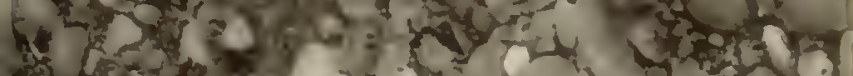

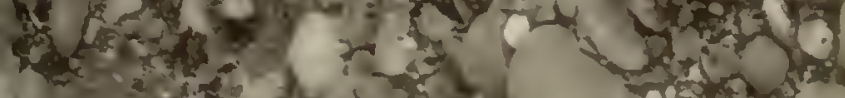

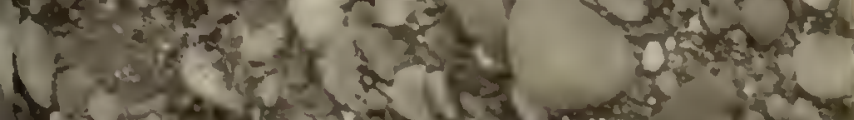

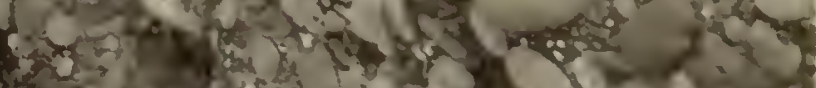
$30, x^{2}$, th

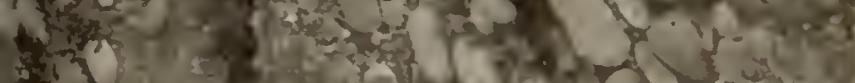

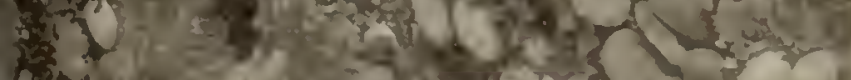
a. $\rightarrow-3 \times 10$,

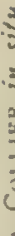

w

2

$=$

C.

(2)

L

$\leq 2$

0

if $\%$

i. $\div$ ?

i. $\div$

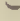

$=$

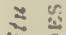

$\therefore 2$

. $x$

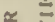

$\div 2$

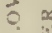

단

2
3
3
5
0
3
5
$=1$ 


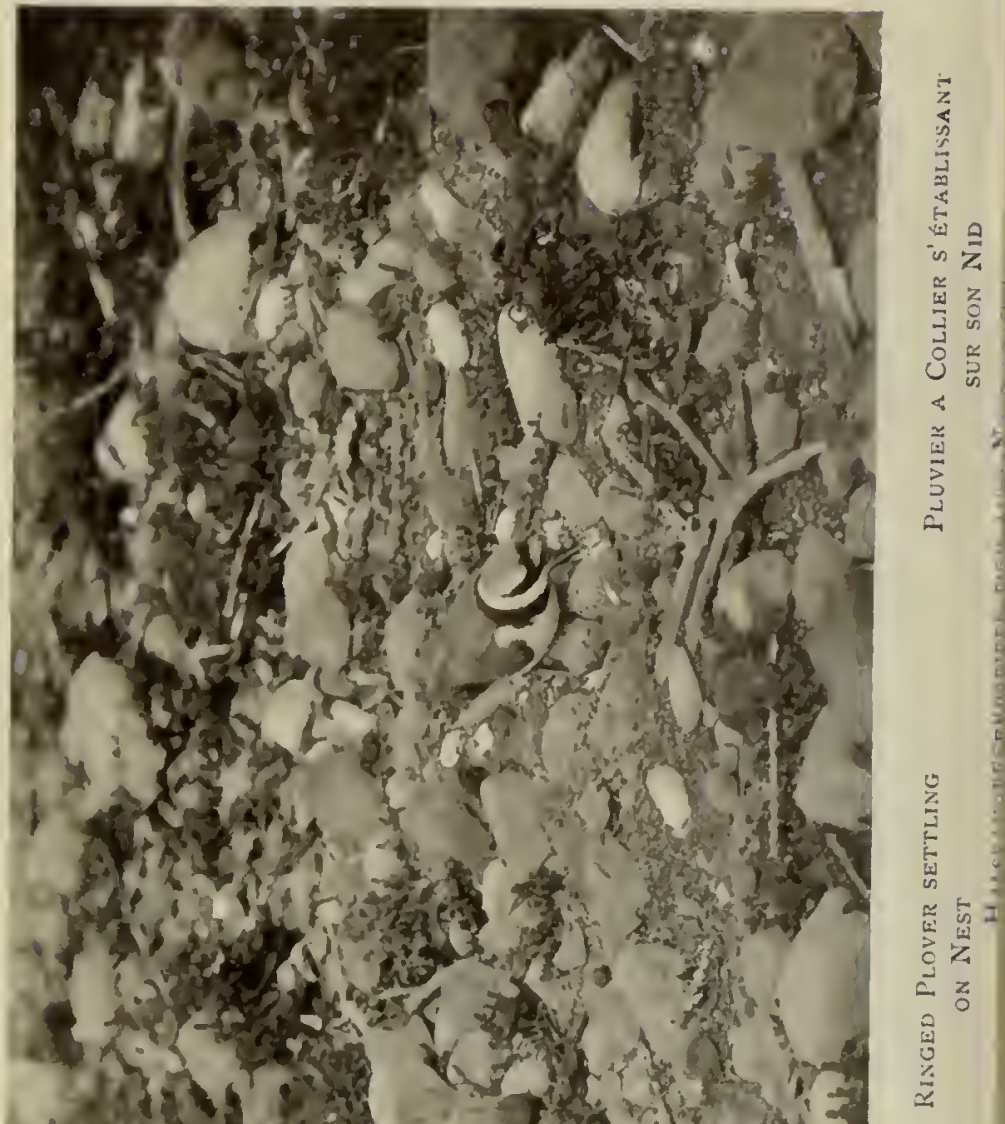




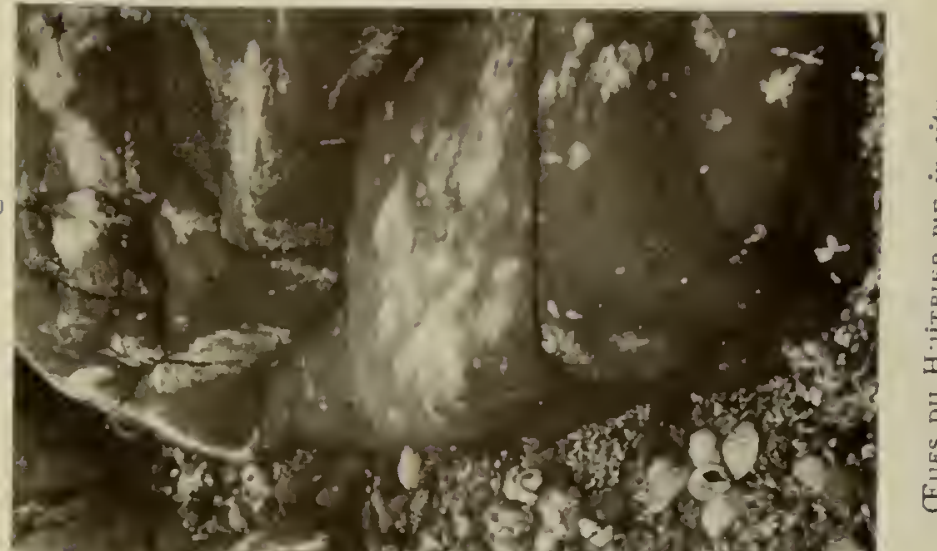

3

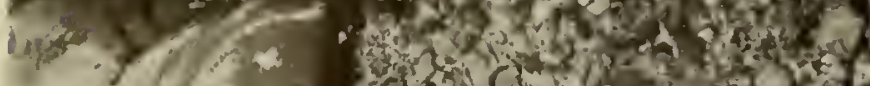

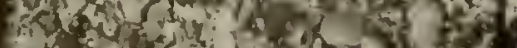

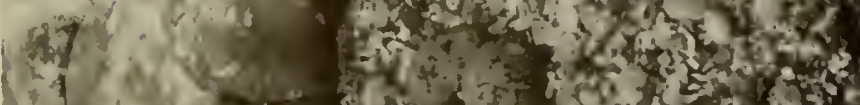

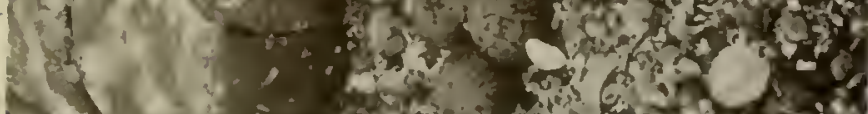

Q

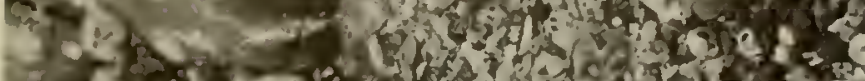

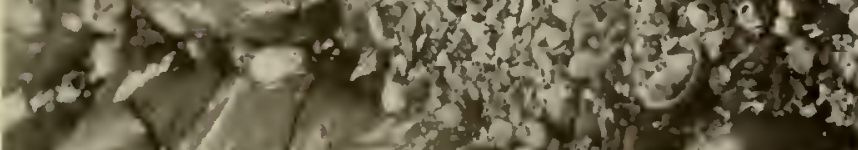

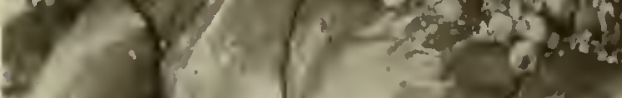

1

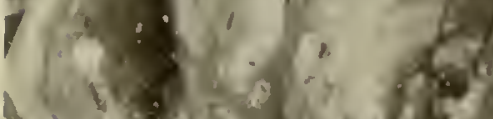

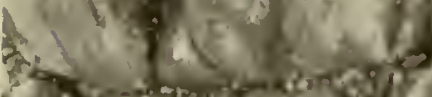

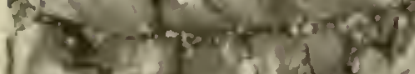

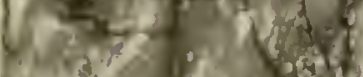

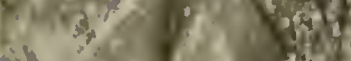

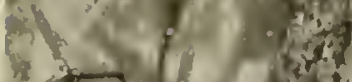

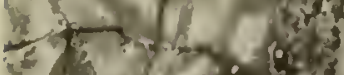

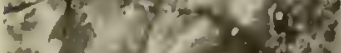

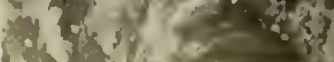

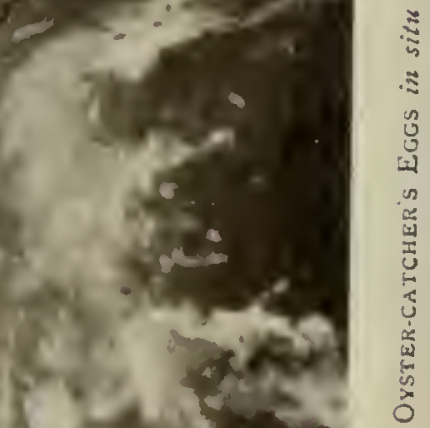




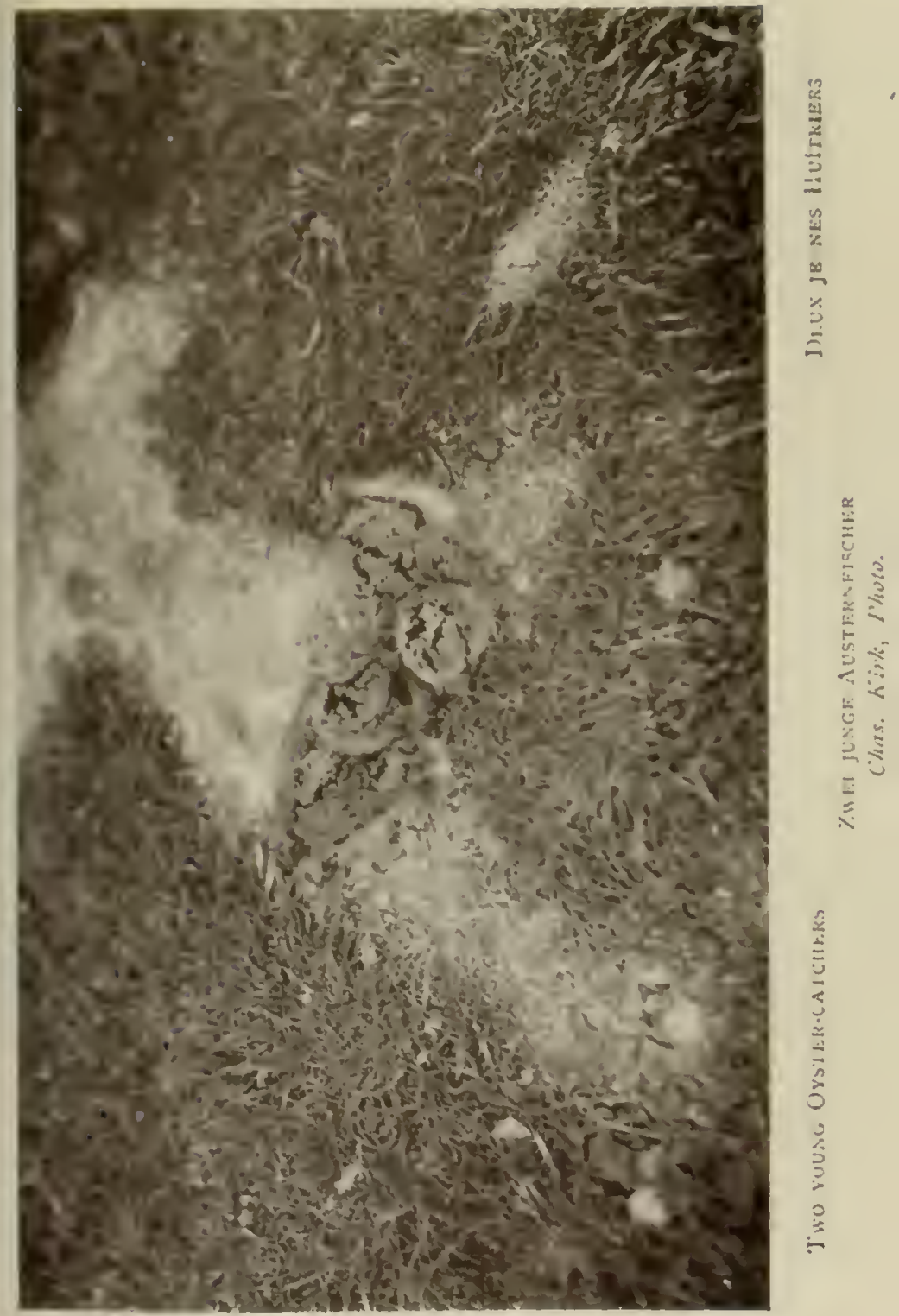




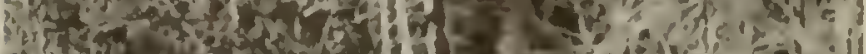
- bitio

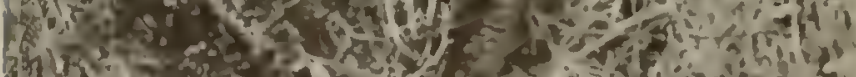

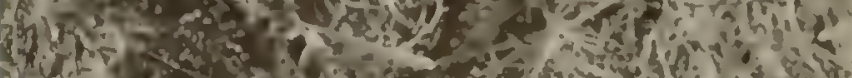

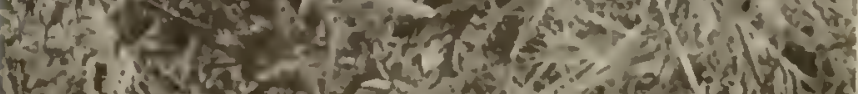

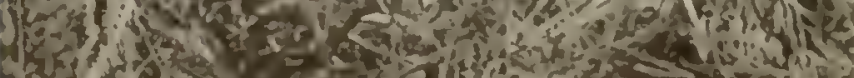

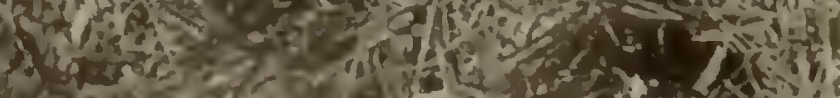

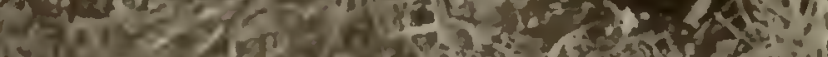

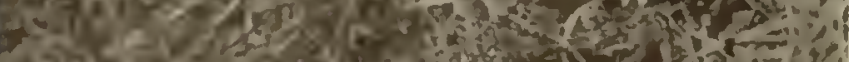

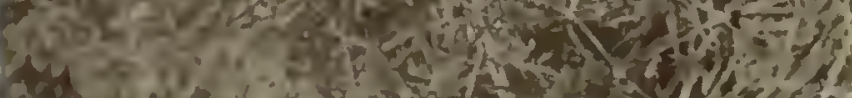




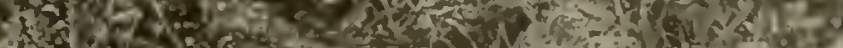

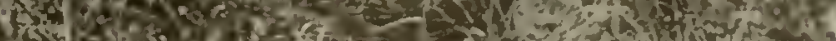

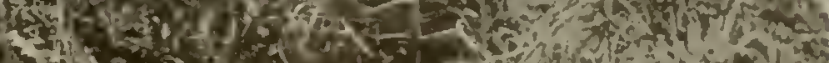

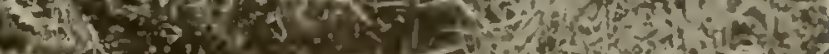
1.m.

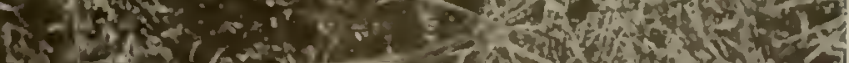

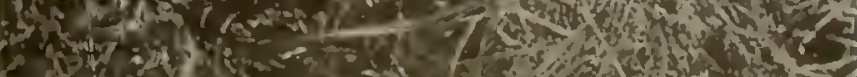

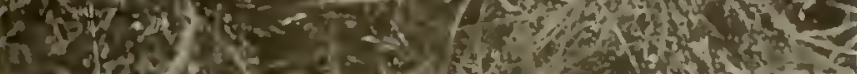

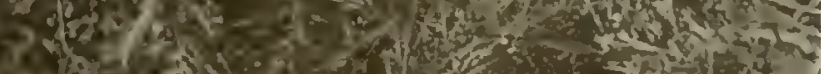

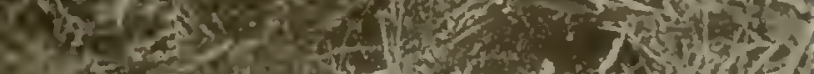

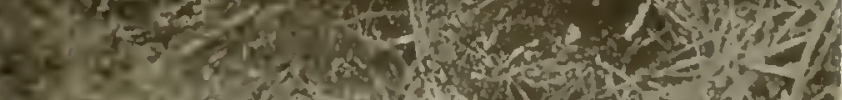

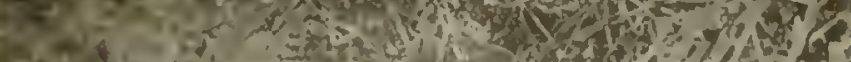

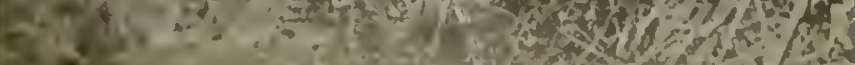




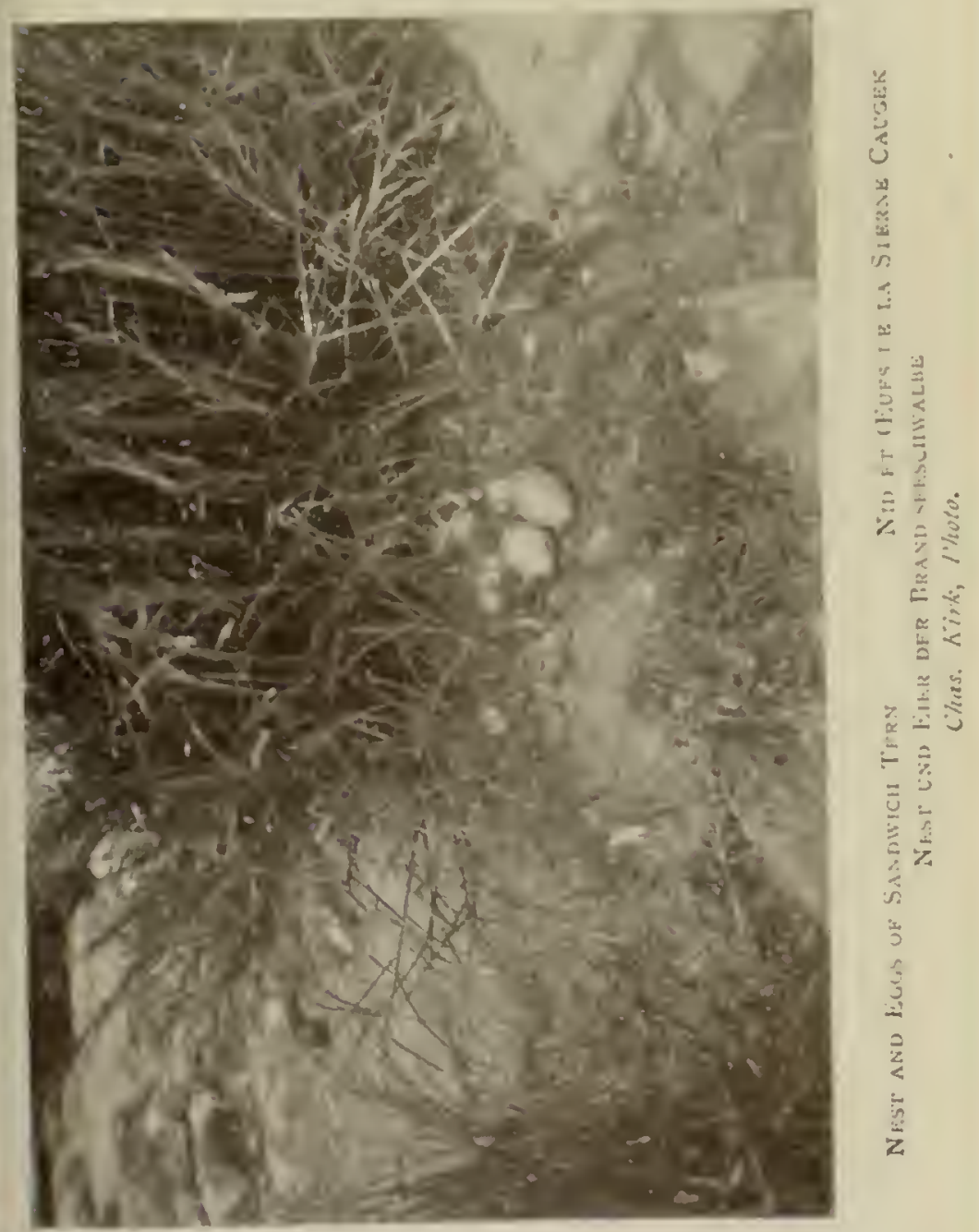




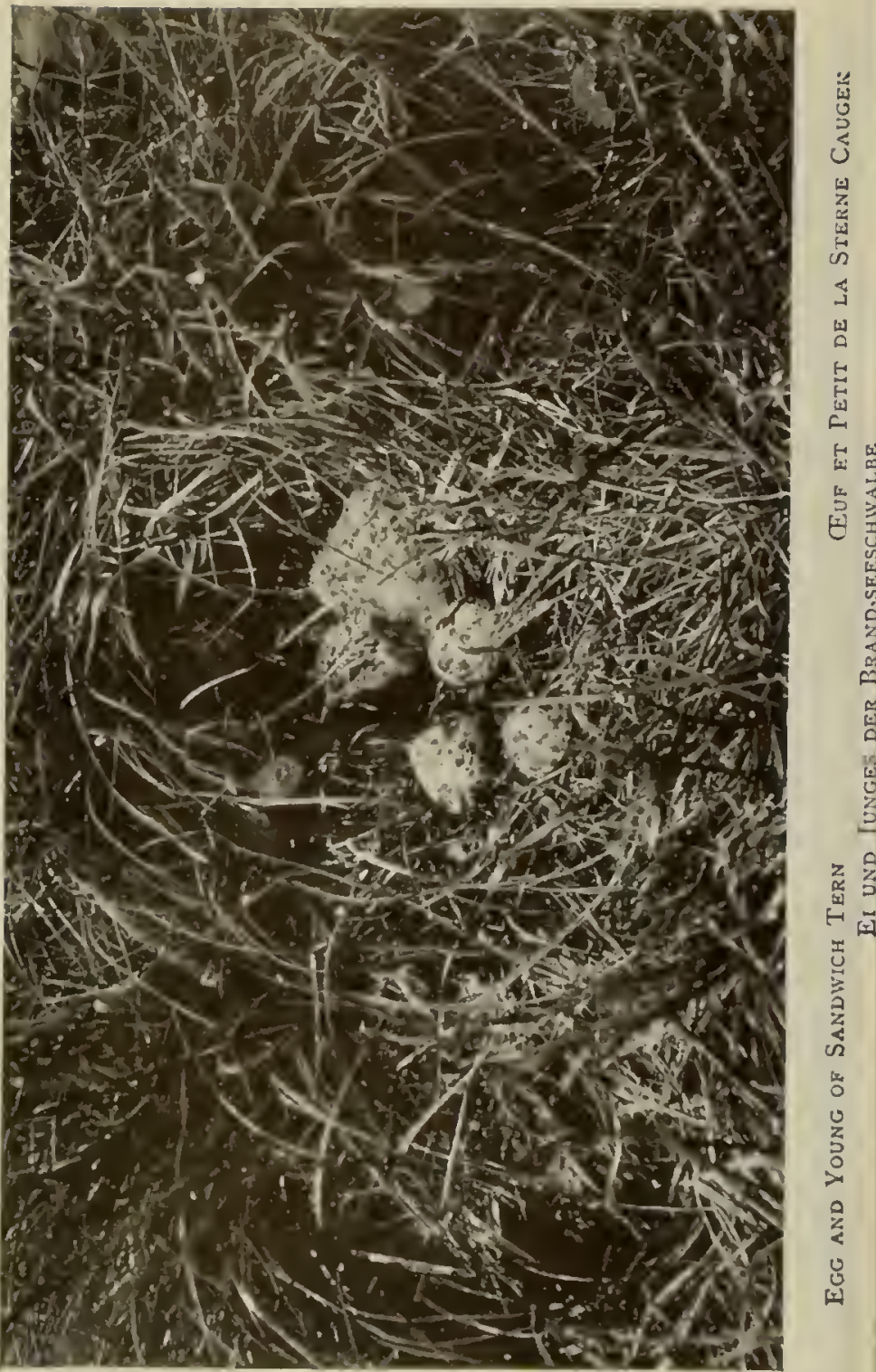




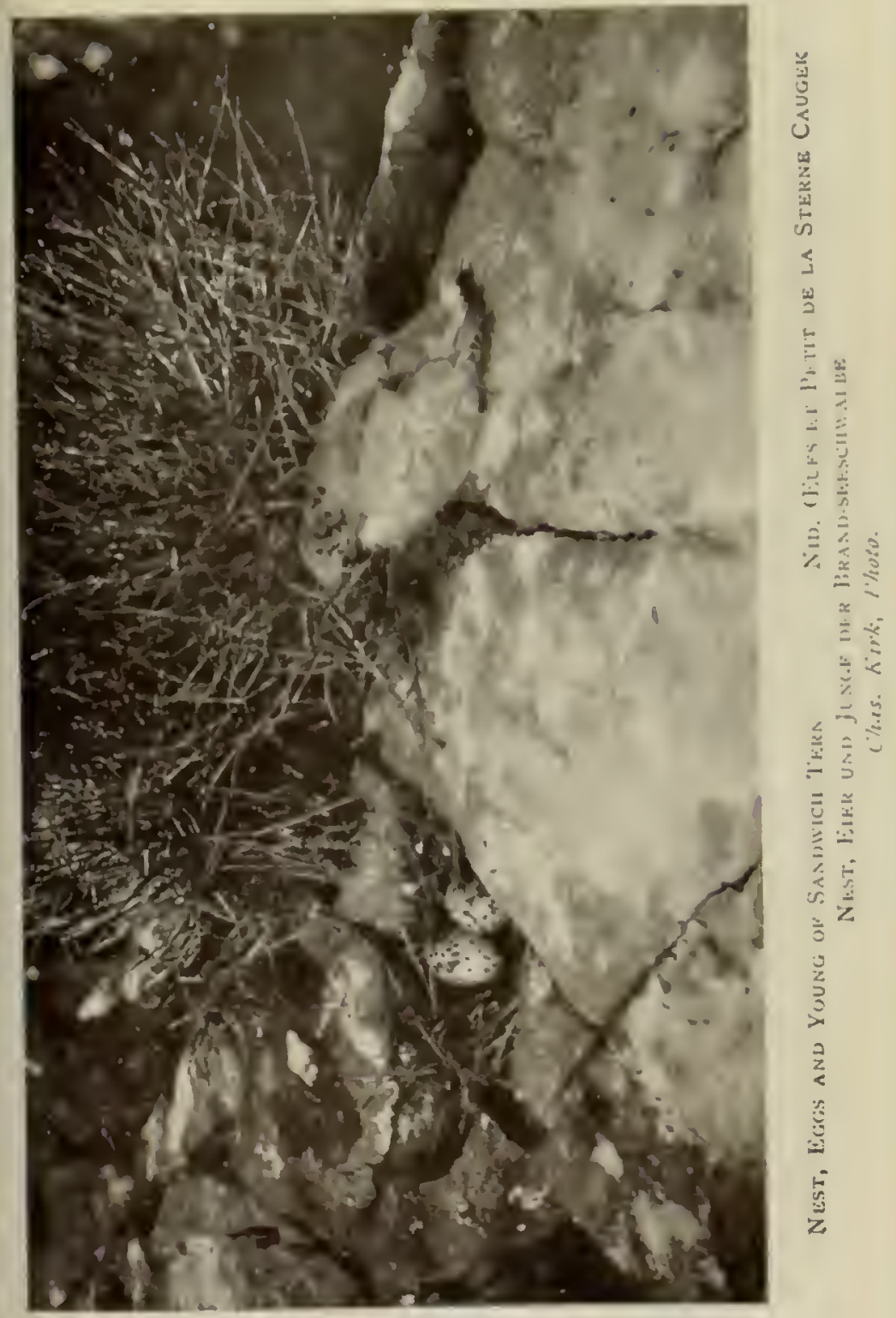




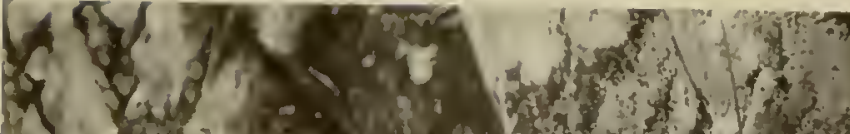 \\ 1. $y$ o $(1)$

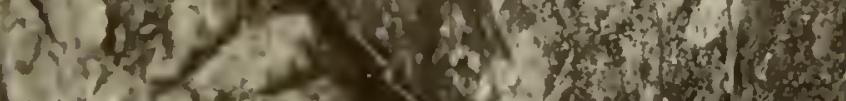 $3,7, y, 4,4$

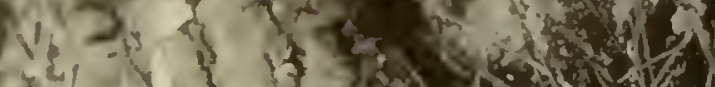

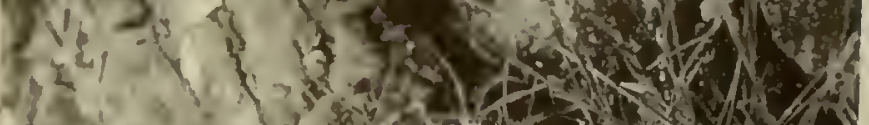

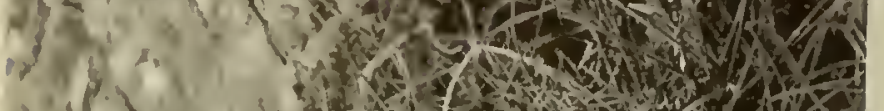
$240(0)$

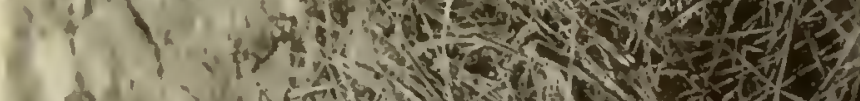

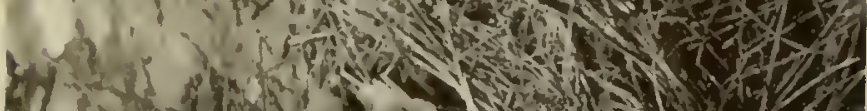

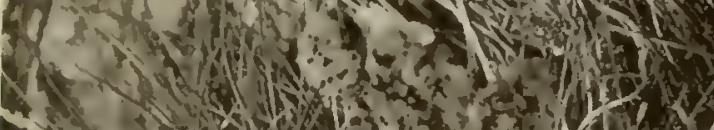

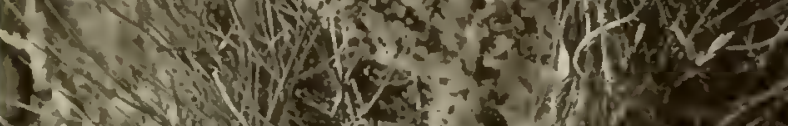

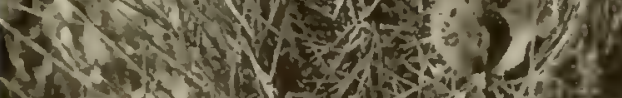
2013 x. X T b. ros nescos $x+3$ $x=\sin ^{3}$ $\therefore x^{2}$

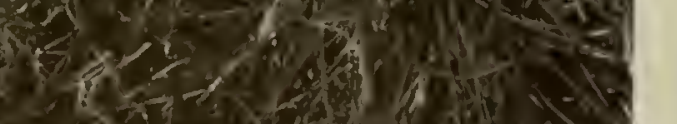

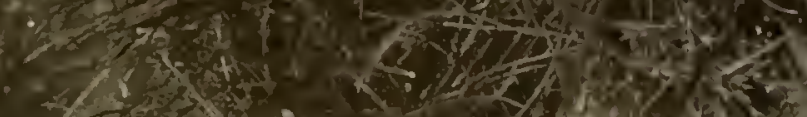

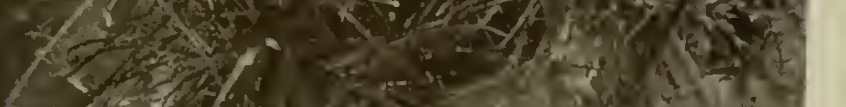

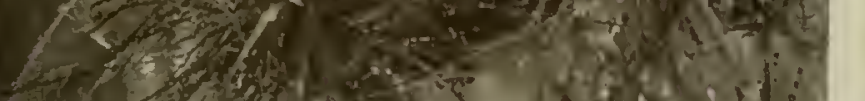

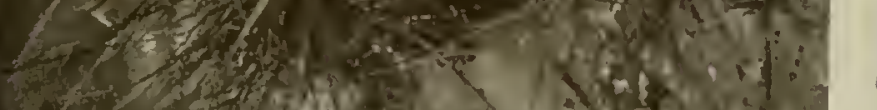

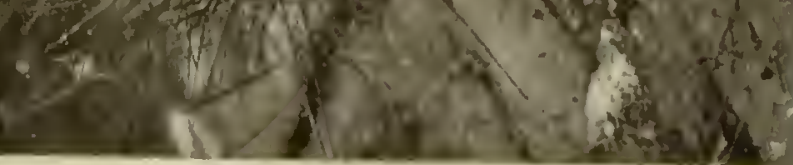




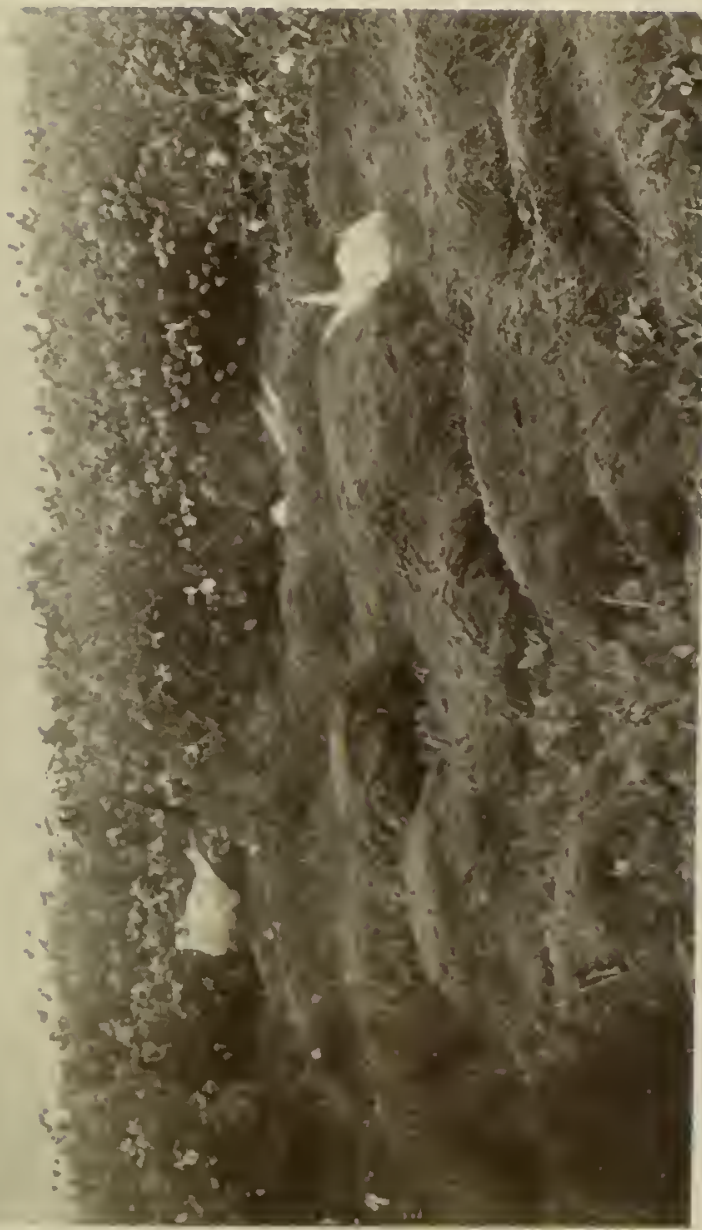




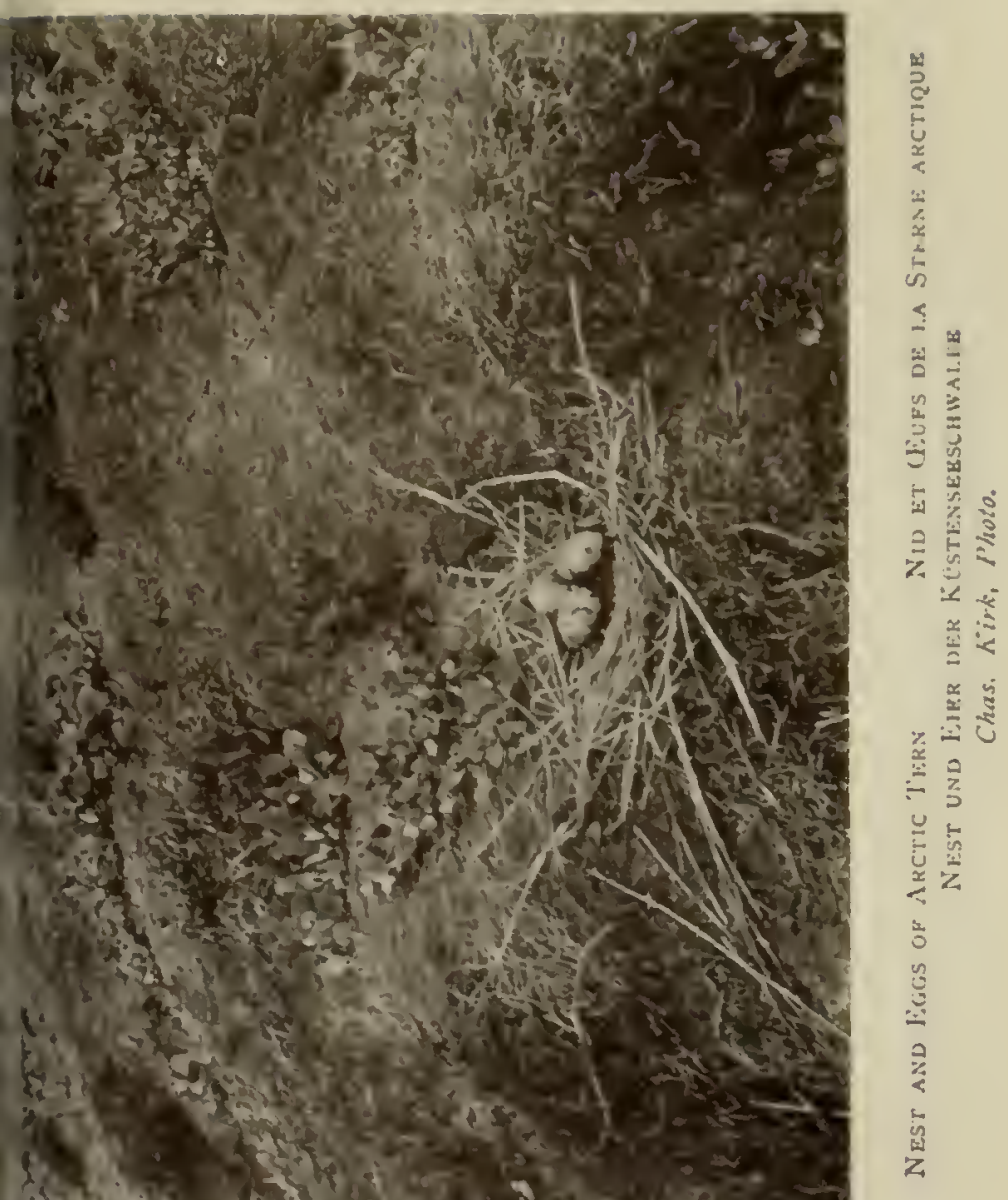




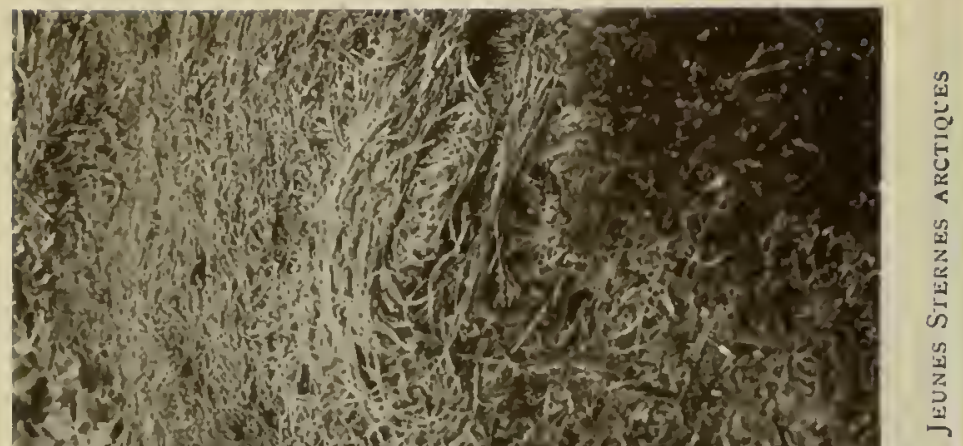

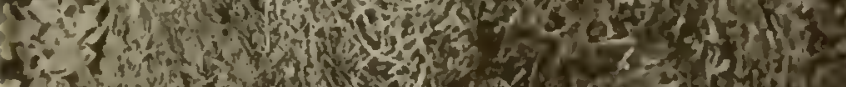

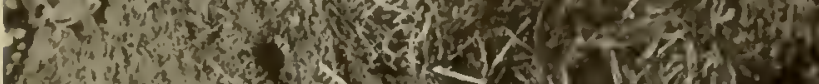

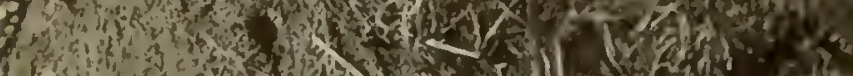

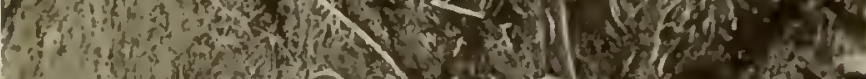

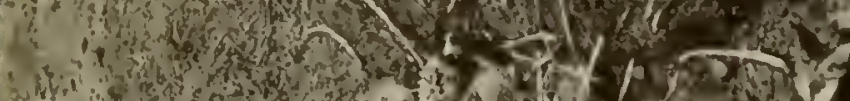

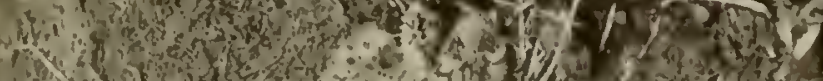

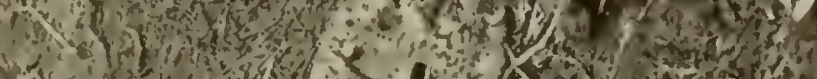

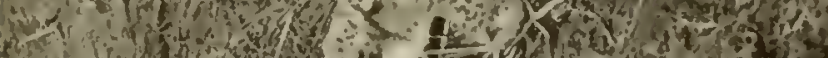

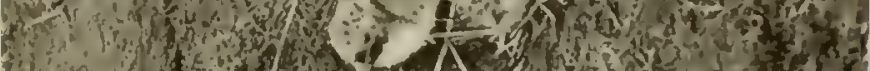

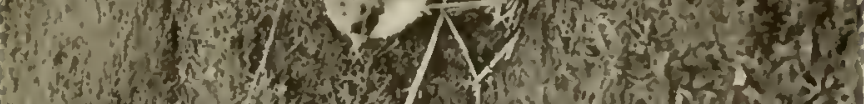

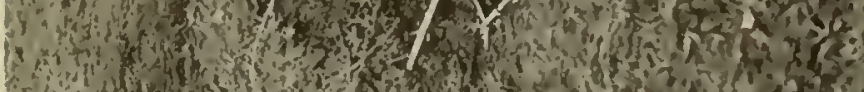

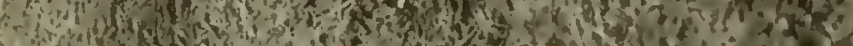

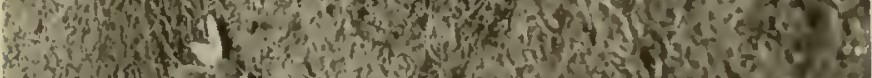

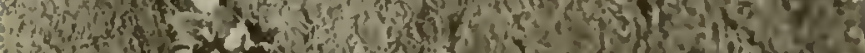

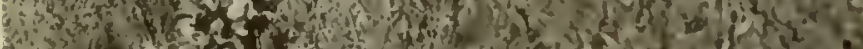

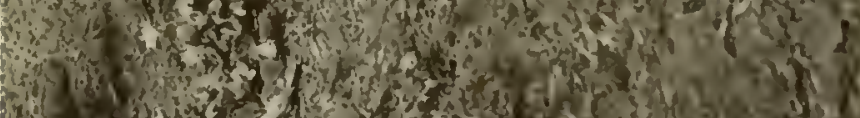

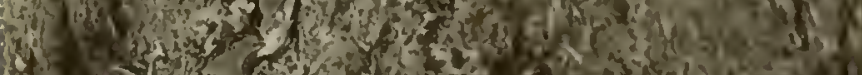

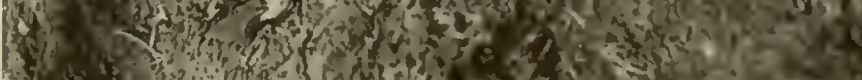

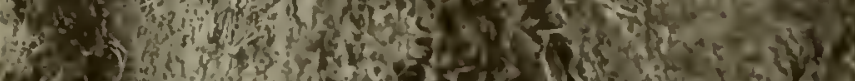

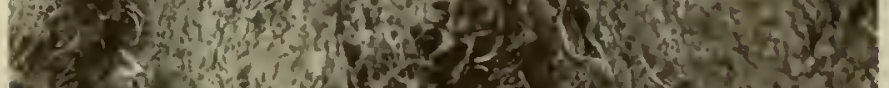

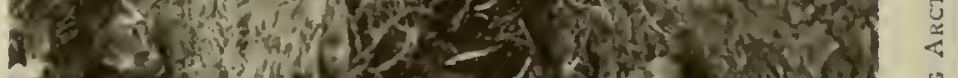

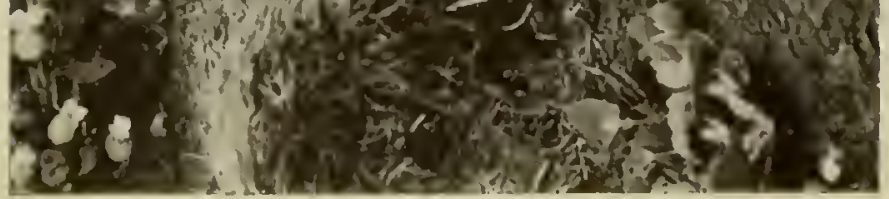




$$
4
$$




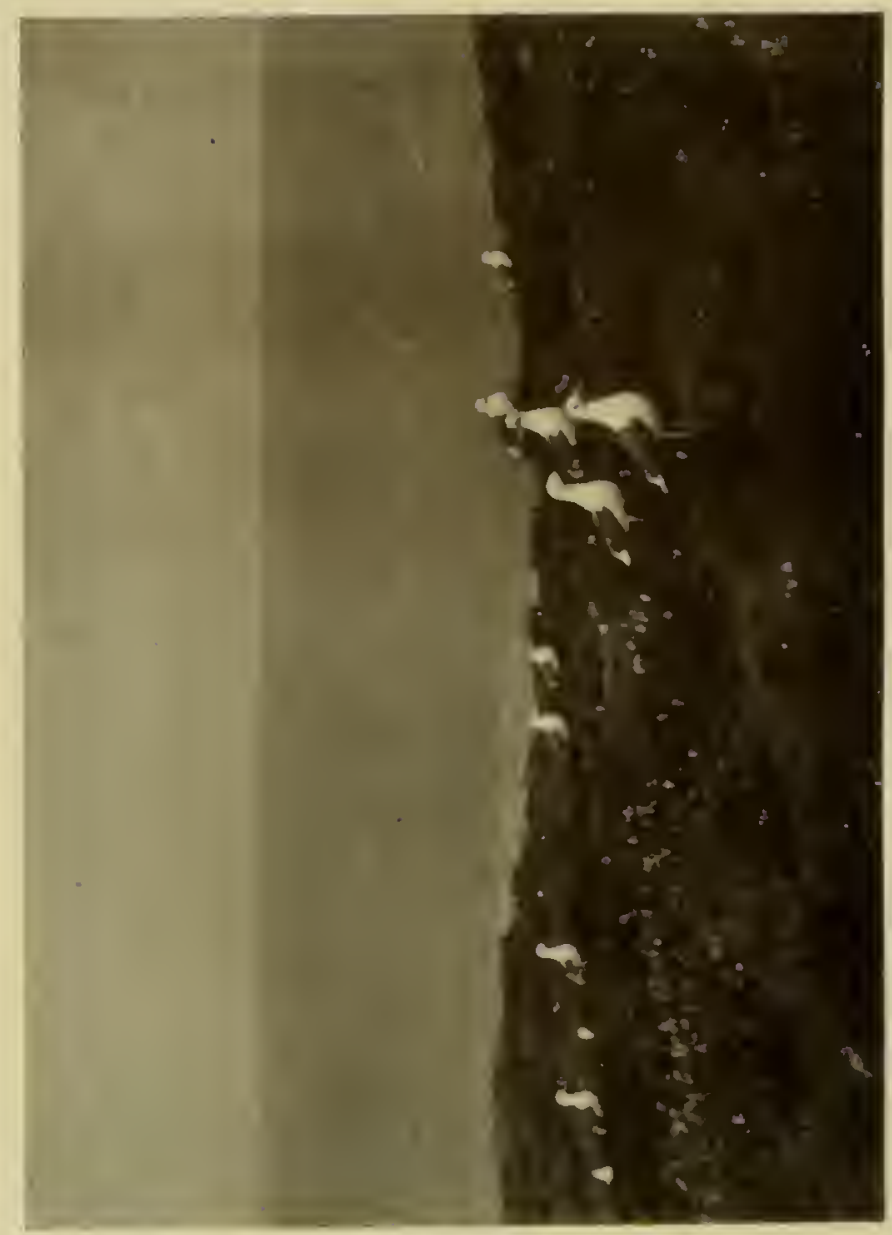

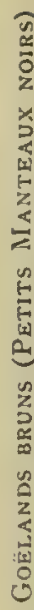




\title{
Some Notes on the Birds
}

OF $\mathrm{WHICH}$

\section{PHCTOGRAPHS APPEAR IN}

\author{
THE FOREGOING PAGES .
}

\section{DY \\ GEORGE GIRDWOOD.}

Capercaillie. - The Capercaillie is much the largest of the British gnme birds, the male attaining to the lengtl of 35 inches, the female veing some ro inches shorter. 'The former is a very' handsome hird, dark slate grey in colour, with each feather finely vermiculated with white. It is possessed of an extremely powerful bill, curved in shape, and has a habit of distencing the feathers of the 1 hroat, the combination giving to the hird a some what fierce look, which is however alto. gether belied by its nature and hahits, which ntuch resemble shose of the black zronse. "The colour of the female is chest'ut, barred with black, the tips of the feathers being touched with white. This apecies was at one time indigenous 10 Great l3ritai 1, but hecame extinct. It was, however, re-introduced to Scolland sume 50 years ago, and has within late years greatly increased in numbers, and in its range; oocurring now frequently in counties much farther south than that into which it was re-introduced. The food of the Caper. caillie consists very largely of the needles of the Scots fir, a diet which imparts to its flesh a strongly resinous flavour. In dissect. ing a mature male, it is not uncommon to find its crop distended with an enormous mass of these needles, so hard and dry as to cause adiniration of the extraordinary digestive power which can reduce and assimilate such unlikely material. This hird also feeds on berries and acorns, and the young shoots and buds of a variety of tuees. The Capercaillie is polygamous. and in the hreeding se.son the males are said to indulge in severe fighting, at which time they may he easily" approached. The nest of the Capercaillie consists of a mere scraping in the ground in which are laid from five to eight eggs, or in the case of older females from eight to twelve, though as many as sixteen eggs have heen found -in the last case probahly the product of two heus. They resemble the eggs of the hlack grouse, being of a dull cream ground colour, spotted with redulsh brown, and are rather over $\mathbf{z}$ inches in lenzth.

Cormorant. - The Cormorant is a resident species, breeding in all suitahle localities round our coav line. except in the north and north-west, where it is superseded by the Green Corniorant or Shag. This hird is easily identified. Most of our sea fowl are light in colour: the Cormorant, on the other hand, is a dead black, though in the hreeding season this is varied by a slight metallic wash of bluish hlack, and at this season also hotl sexes assume a series of white nuptial plunes, extending from the crown down either side of the neck, and also a large white patcl on each thigh. The length of the Cormorame is 32 inches. 
In flight the Cormorant is a heavy hird, and though once on the wing it moves with considerable rapidity, it yet gives the observer the appearance of being heavy, and its flight only sustained hy much exertion. The hird is a most rapacious feeder. Its food consists entirely of fish, which it captures by diving, and in its pursuit of its prey below the surface it is itself fish-like in the rapidity of its movement and the length of the time it remains submerged. After the period of feeding and its consequent diving a very favourite attitude of the Cornorant is to sit upon a rock, or even on a huoy, just ahove the reach of the waves, with its wings extended and apparently in a state of repletion.

The Cormorant hreeds in colonies, most frequently on rocky ledges on the sea coast, but often selects what appears so unusual a site as the tops of high trees, where it breeds in conditions similar to those of the Heron. The eggs are usually three in number, and are of a pale blue ground colour, though this is covered with a chalky white coating which quickly hecomes soiled and discoloured. There is something weird in the appearance of the Cormorant, with its staring light green eye, strong bill with its sharp book at the end, its unfeathered face, and its snake-like neck, and its general repulsiveness of appearance, no douht, was the reason of Milton selecting its description as similar to that of Satan. A visit to the breeding-place is interesting, hut unsavoury. A peculiar bahit in its feeding of its young is that the young bird thrusts its head down the throat of the parent, which regurgitates the balf.digested fish it has captured.

Crow, Carrion.-This bird is a resident, though in winter its numbers are enormously increased by an autumn invasion from the Continent and Scandinavia. Of the Carrion-Crow very little gaod can he said, while it is unquestionably the source of much trouble and loss to game preservers. In size it is slightly larger than the Rook, which it also resembles closely in appearance, the chief dis. tinctions between the two hoing that, while the Rook is always gregarious, the Carrion-Crow is a solitary hird, and the latter has at all ages the face feathered, in contrast to the Rook, which in its second year loses the facial feathers, presenting that rough and uncouth appearance which is so faniliar to all.

The Carrion.Crow has deservedly earned persecution, with the result that it is now a shy bird and to be found in any number only in the more retired and inaccessible parts of our islands, where it resorts to hreed. The nest is placed either in crevices amongst rocks, or perhaps more frequently in the hranches of a tree growing from a cliff face, though now and again an odd pair may' be found nesting in an unexpected situation, such as the corner of 2 coppice, quite near to human habitation, though in such cases it is unlikoly there is a gamekeeper near. The eggs are four or five in number, of a bluish green ground colour, thickly hlotched, spotted and streaked with olive brown. The Carrion-Crow is the nearest relative of the Hooded Crow, with which it not infrequently interhreeds, the young in such cases partaking of the characteristics of one of the parents-that is, appearing either all black, as the Carrion-Crow, or with the plumage of the true Hooded Crow.

In colour the Carrion-Crow is hlack, with a metallic lustre of dark steel blue, which is particularly apparent in the spring months; its length is about $\mathrm{g} g$ inches.

Cuckoo.-This well-known bird is perhaps from one point of view the hest known of our summer migrants, its note being eagerly listened for each year by many as the harbinger of that pleasant 
season which comes with April. Volumes of great lengtb might ba written rogardiog the Cuckoo and its liabits without exhausting its history, but in these short notes one can touch ouly on one or two alient points. First aud foremost is of course the parasitical habit nf the Cuckon, which thrusts the burden of its fanily upon other hirds. The female Cuckoo lays her egs upin tlie ground, and lifring it in her bill places it In the nest of another bird. In our country the hleadow-l'ipit, Hedge-Sparrow, and the l'ied Wagtail are the species most usually victimised. When the young Cuckoo is born it is blind, hut is possessed of an instinct which teaches it to -ndeavotir to eject whatever else be in the nest beside it, and this effort it exerts unceasingly until eggs or young are thrown out and it is left the sole occup.int.

The Cuckoo is a handsome hird, closely resembling in appearance the Sparrow. Hawk, for whicl it is indeed not infrequently taken. Above the general colour is asliy blue, the hreast is white, barred with black; while in the fernale on the breast there is a wash of rufus colour which still further leads to its resemblance to the Sparrow.Hawk. The bird has been the subject of much study by ornithologists from the time of Dr. Jenner, of vacciration fame, up to the present day, and the conclusion has been senerally arrived at that one of the reasons for the parasitical habit of the bird is that the males greatly exceed the females in number. One German authority states be bolieves that the fenale Cucko, lays up to as many as 17 or 20 eggs, but as, of course, each is placed in a different nest any definite conclusion on this subject is most difficult to arrive at. The old Cuckoos leave our country for their winter baunts in Africa, Persia, and elsewhere at the end of July, and not the least interesting speculation connected with the migration of birds is that with which one wondersliow the young Cuckoo without guide, philosopher, or friend fiods its way later in the year to these far distant shores. The egg of the Cuckoo is very variable, light blue egrs being occasionally found, but the ordinary type resembles closely that of the Meadow-Pipit, though someuhat larger in size than that of the latter bird. The Cuckuo is insectivorous, is par. ticularly fond of caterpillars, and is stated to be the only bird which preys upon the bairy variety of this insect.

Dipper, or Water-Oueel.-The Dipper, or, as it is known in Scotland, the Water Crow, is a native bird, distributed over the British Isles, more numerously on the mountain streams of our higher lands, though it is also to be found on the moorland streanis of Devonshire and elsewhere. In appearance the Dipper resenubles a large Wren, being of the same build and carrying its tail in the same perky, upturned fashion. In colour this bird is of a dark sooty brown, sliowing up in the surroundings where is is to be observed as a distinct black, save on the breast, which is pure white. Its length is seven inches.

This bird is one of our most interesting on account of the peculiarity of its babit, disassociated as it is with anything in its appearance wbich wculd lead us to suppose it possesses the powers it bas. As we have stated, it resembles the $1 W^{\circ}$ ren in its appearance, but it is really a water bird, under the surface of which it cbtains its food. It feeds chiefly upon the Caddis. $11 \mathrm{c}$ im and the larva of water insecis: these it finds at the bottom of pools into which it walks, or, slipping off the side of a stone. disappears horizontally beneath the surface. How the Dipper nianages to keep itself under water in places such as the swift running part of a stream, where it may often be chserved, is somewhat of a nystery, yet it does so, and 
the fortunate observer who may see the Dipper enter the water may with great caution approach suffciently near to see it moving along the bottom actively engeged in its earch for food. When it so desires it rises, cork.like, to the surface, and, taking wing, usually flies in a straight line up stream to the nearest stone atfording a resting-place above water. Like most of our native birds, the Dipper breeds more than once in the cours of a season; the first nest may be found in favourable seasons early in April. It resembles the nest of the Wren, though it is, of course, much larger, while the aperture is lower down in the side. It consists of a large ball of moss felted closely together, and is lined with dry leaves, frequently those of the beech, and contains four or five eggs, pure white and of a rather pointed shape. They, before being blown, possess a delicate pinky tinge, due to the translucence of the shell and the presence of the yoke within. 'The nest has, in the writer's experience, been always placed above water in such a position that the young, if emerging before flight, would fall into the water. It is most usually placed in the crevices of a rocky bank, though occasionally in the roots of a tree projecting from a similar situation. The song of the Dipper is very musical, and heard in the young months of the year and in its usually exquisite surroundings has a charm all its own.

Duck, Common Eider.-This bird is resident with us throughout the year, in winter its numbers are added to by migrants from the Faroe Islands, and also from Scandinavia, but a considerable number instead of proceeding north in the spring remain with us to breed. On the well protected Farne Isles they breed in numbers. Their favourite haunt is further north, numbers frequenting the Hebrides and larger numbers the Orkneys and Shetlands. In the latter Isles, it is not an uncommon sight to find eight or ten Eiders on a small out-lying Islet.

The well-known down of this bird is a commercial commodity, and they are accordingly afforded much protection in Iceland and Scandinaviz, where the down is regularly gathered, but the bird does not occur in our Islands in numbers sufficient to enable this to be profitably done. The female Eider like all the others of the duck tribe lines her nest with the down pulled from her own breast, this down is of grey colour, centred with white, and a very considerable portion is employed in the construction of each nest. In the centre of this warm and comfortable nest, are to be found from three to five eggs of a greenish grey colour. The female Eider is very tame at the nest, often sitting until actually touched. The male Eider is a particularly handsome bird, some 23 inches in length, the plumage of the back is pure white, and the crown of the head of a rich glossy black, the sides of the head and neck are white, washed with exquisite Eau-de-nil. The breast is white, washed with a delicate roseate cream, ending in a clearly defined line, where it meets the under parts of the bird which are of a rich black. The male Eider has in the breeding season a most peculiar call, resembling the coo of a dove, though much harsher in tone. The food of the Eider consists of shell-fish and its digestive powers may be described as tremendous. In dissecting this duck a large quantity of whelks may often be found in its gizzard, entire, including the shell. They are there ground to a rough, gritty powder by the powerful action of this organ, and pass to the stomach, where the uutritious part is assimilated and from which the ground sliell is ejected.

Duck, Common Sheld.-This bird is our most brilliantly coloured duck, its plumage being in broad patclies of pure white, 
jetty black, and ricb cbestnut, wbile its bead is of a ricb dark metallic green. Its bill and feet are brilliant red, wbile the bill of lie nale has a basal knob of the same rich brilliancy. Its length is 22 inclies.

The Sheld-Duck is a resident species, and is becoming less unconmon of recent years. The djustment that nature makes for the preservation of species is shown by the nesting babit of this Uuck u.jil brilliant plumage, namely, to resort to rabbits' burrows. WV hen in the open its colour of reveals it to the prying eye, but in the dark seclusion of its underground home, which it enters and energes from in the inost careful faslion, it is lot to be easily discovered. At a distance of some fuur or five feet from the entrance of elie burrow the Shelil-1)uck makes her nest of down, pulled from her own breast, aud in this most exquisite of nests she lays from 12 to even 16 large creany-coloured eggs. It is a most interesting sight to sea the parent birds bring down tbeir young brood from where they have been born to the water. It is 2 time of great anxiety", as tlie duck. lings are inost defenceless, and the ingenuity and patience of both parents and young in overcoming tbeir difficulties is vel y wonderful.

The food of the Sheld-Duck consists of slugs, worns, and shell. flih, and is invariably obtained on or near the sea coast. "This is nne of the birds which has undoubtedly lienefited by the Wild Bird Protection Act, and it is to be boped its numbers will still contiuue to increase that its beauty may become more common round our sliores.

Flycatcher, Spotted. - This "most familiar bird," so lovingly" written of by Gilbert White, is widely distributed and not uncommon. It is a summer migrant, and may be identified by its persistent habit of perching on the top of some favourite post, from which it ever and again flits out to hawk at a passiug insect, returning to the post only to repeat its flight after a short interval. Save for this habit it is so unobtrusive as to attract but little notice; its lack of song and incouspicuous colouring allowing it to pass unnoticed in many cases. The nest is placed in a variety of situations, the most favourite being possibly a recess in a tree trunk, where a brancb has rotted out, or in the cleft where a branch springs from the main stem. though a clump of fern growing out from a wall or a hole in the wall itself are sites ofien selected. The eggs, four or five in number, are usually. of a pale green ground-colour, prettily blotched with a light brown, thougl occasionally a clutch may be observed where tbe ground. colour is of decided green, witb distinct spots of red-brown.

Greenfinch. - Tbe Greenfinch is resident in Britain throughout tbe year, and is not uncommon. It is a beavily built bird, about six inclies long, and is possessed of a very tbick and powerful bill, which it uses with effect upon tbe seeds on which it chiefly feeds, tbougb, like mosi of our other small birds, it feeds its young chiefi. upon insects. Tbe general colour of tbe Greenfincb or Green Linnet, as it is sometimes named, is olive green, but this is relieved, particu. larly in the male bird, by, on wing and tail, a ligbt and brilliant sellow, making the bird when in flight quite brilliant.

Tbe favourite haunt of this species is tbe hedgerow, small coppice, and garden, where its feeble efforts at song are to be heard in the spring montbs. The nest is placed often in tbe hawthorn hedge, sometimes in small fir trees, but is also to be found in whins and iry. covered walls, and it is not an unusual thing to find two or even tbree nests in comparatively close proximity. The esss are four or five in number and of a pale greenish ground tint wish small spots of reddisb brown. In size they aro just larger tban tbose of tbe 
Common Linnet, which in appearance they closely resemble, though there is a great variation in size in the Green Linnet's eggs, with the result that occasionally the sizes overlap-that is, eggs of the Green Linnet may be found smaller than eggs of the Common Linnet, and identification is impossible without ob-ervation of the parent bird.

Gull, Lesser Black-backed.-This bird, a resident in Britain throughout the year, is numerous and widely distributed along our const-lines. It is a large and handsome lird, white in colour but with, as the naine denotes, b.ack and wings of blue.black colour, and with a large and powerful beak. The young do not become mature until their fourth year, and are until then of a dull white colour, speckled with grey. "The Lesser Black-backed Gull has few, if any good qualities, nay, is indeed both thief and murderer. They nest most usually in the vicinity of other sea fowl, and are ruthless robbers of the eggs and young of such species as Guillemots and Kittiwakes, selecting a nimment when the parent bird is absent, to make a dash and seize their prey. Their nest is a slight structure of dry grasses, placed upon the green slopes of the cliffs along our coast-line and islands, but cccasionally they will select an island on some fresh.water lake, or even the heaiher of the open moorland. The eggs are three in number, somewhat variable in shade, but usually of a greenish stone ground-colour, spotted and blotched with grey and black, and are not to be distinguished from those of the Herring.Gull, save by identification of the bird as she rises from the nest.

Kestrel, - Ihe Kestrel is the commonest of our British hawks, and is a harmless inoflensive bird, but alas! to the indiscriminating eye of the ganie-keeper, 2 hawk is a hawk, and the mouse-loring "windhover" has oft-times to fall the victim of his gun. The food of the Kestrel consists almost entirely of mice and beesles, and it is while in pursuit of the former, that it may so frequently be seen hovering high in air, there poised for a few seconds absolutely motionless, ere it darts down to seize the mouse it has been watching. The Kestrel is a handsome bird, the general colour above dull chestnut, with a black spot in the centre of each feather, in the male bird the head and neck are steely blue, as is also the tail, the latter being tipped with white; the throat is buff, the breast and undersides reddish fawn, streaked with black. In the female the blue of the liead and tail is absent. The Kestrel is partially migratory, but is found in Britain throughout the year. In the winter it will leave the high lands and descend to avail itself of the less severe conditions of the lower lying parts. A number migrate to the continent while an immigration of a certain number takes place to the British Isles from more northern countries. The Kestrel nests in cliffs, old ruins. or even in the old and deserted nest of a crow or wood-pigeon. The eggs, four, five, or even as many as seven in number, are white in ground colour, but often this is so thickly blotched and spotted with brownish red as to be barely visible.

Oyster-catcher. - This bird, known as the Sea Pie or Mussel Picker, is a bird of brilliant plumage, and though not uncomnon, is in only a few localities abundant. It is resident and breeds with us, but its numbers are augmented in winter by migrants from breeding grounds further north. 'I'lie length of the Oyster-catcher is some 16 inches, the upper parts are of a rich glossy black, con. trasting sharply. with the white of the lower parts, and giving the appearance which has suggestrd its mame of Sea Pie. The bill is some three inches in length and of a briglat blood ied, the 
legs are somewhat long in relation to the size of the bird, and are of a purplish orange. It is to be questioned whether the bird actually: preys on oysters, but it is without duubt that both the muscel and limpet form the staple of its food, wbile small crustaceans and sheltfish of nither kinds atso form part of its diet. In the Shetlands it is particularly numerous, and it is there known as the Shelder. On all the coast lines of these rocky islands it is to be found breeding, never in colonies, but often several pairs may be found comparatively near each other. The egrs are ino or thee in nunber, tbe ground colour, clay, spotted, blotelred and streaked with black, and with grey underlying shell nurkines, the egg lias a slight gloss. "This bird is often to he found in subll parties in wuter, and they are very wary, unally seuding out a loud piping whisıle before the advance of the sportsman within cunsliot, and so warming all neighbouring fowt that danger is near.

'The nest of the Oyster-catcher consists of a slight deprestion on the ground, often in shingle, is shallow as though shaped hy the breast of the bird and is invariably lined with pieces of wlite shell. A notable feature of the nesting of this bird is that it makes several of these depressions lefore finally fixing upon one. There mock nests are not lined like the final one, but are often the guide to the vicinity of the true nest.

Pipit, Mradow.-1he Meadow.Pipit, or Tit-Lark is common and uidely distributed over the whole of the 13ritish Isles. In appearance it resembles the Skylark, but is smuller in size and darker un colour. $I_{t}$ is pre-eminently the small hird of the moorland, where it nests, carefully concealing its eggs within the shelier of a tussock of grass. The egns, four or five in number, are of a dull bluish white in ground. colour, but are so thickly hesprinkled with spots and freckles of grejish-hrown as to leave the ground-colour barely visible. This is the bird in whose nest the cuckoo frequently places is ege; and it is indeed an incongruous sight, that of the two \% it. Larks teeding the young monster palmed of upon them, which, even before fully grown. is in bulk much larger tban both its foster-parents together. This species is much preyed on by the Merlin, 10 whose dash, in the absence of cover on the inoor, it falls a somewhat easy victin. While resilent with us throughout the $\mathrm{y}^{\mathrm{e}} \mathrm{ar}$, it descends during the winter months from the high moorland to lower-lying and less inho-pitable parts.

Plover, Ringed.-This bird, known also as the Ringed Sand Plover, and in some localities as the Sand I.ark, is resident with us, and is widely distributed breeding along the coast line in many localities, as also round the consts of the Hebiides, Orkneys, and Shetlands. It is an insect eater, and finds a plentiful supply of fo d, particularly in the summer months upon the insect life present on the beach.

In colour the Ringed Plover is of a light brown on the upper parts, the forehead pure white, divided from the light brown at the back of the head by an intermediate band of black. There is also a band of black round the upper part of the breast, while the under parts of the bird are white: the tail and legs are orange, and the whole appearance of the bird assinulates in a wonderful degree with its usual surroundings. Ihe length of the Ringed Plover is 7 inches.

During the breeding season in particular, the Sand Lark haunts those parts of the beach rich with yellow sand and covered with sbingle, and were the bird to remain quiet it would be well nigh impossible for the human eje to discover it, but it greets the intruder with a low, pipiug whistle, and trips along with burried 
little steps, usually close to the margin of the sea, ostentatiously showing off with an overdone air of innocence as though the last thing it thought of was nesting. However, in early May, 2 very careful search in the neighhourhood where the hirds are seen will reveal one of the most wonderful instances of nature's protective power. On the shingle, in a slight indentation often lined with small particles of shell may be found the four pear-shaped eggs. In ground colour hright clay, spotted with black, and so exactly resembling the shingle on which they rest that even to remove one's eye for a moment renders it difficult immediately to re-locate them. The photograph gives an instance of how bard these eggs are to find, and when the knowledge is given that the eggs are present on this small pbetograph, this is contrasted with a search along a mile of sea shore it will he evident how well nature cares for her own.

Redstart. - This tird is a summer migrant, arriving on our shores usually in the earlier part of April. The males precede the females by $2 \mathrm{few}$ days, and it is at this period that this species is most usually to be ohserved, as later, after pairing, they hecome shyer, and are then much less in evidence. The male is a very handsome hird, ahove slatey grey, the forehead pure white, the throat hlack, while the under parts are a rich orange chestnut. The tail coverts are also of this chestnut colour approaching to red, and this, com. bined with the quaint lateral movement of the tail, has given the hird its popular name of Fire Tail. The length of the Redstart is between five and six inches.

The Redstart is widely distributed though in no district very numerous. It haunts the old walls surrounding woodlands and quiet country roadways. I ts food consists mostly of insects, many of which it captures after the manner of a flycatcher. In autumn this species varies this diet with seeds and fruits.

The nest of the Redstart is always placed in concealment and is sometimes very difficult to find; holes in old walls are favourite sites, while cavities in old trees and similar situations are frequently resorted to. The eggs are five, six, and sometimes seven in number; the colour resembles that of the egg of the Hedge Accentor, hut is slightly paler; while the egg is rather smaller in size, more of a pointed oval and with a greater polish on the surface.

Ring-Dove.-The Ring Dove, Wood-Pigeon, or as it is known in Scotland, Cushat-Doo is a large and handsome bird. It is resident and now breeds freely in almost every locality, rearing several broods each season, as do most of the genus. At one time it was not so numerous in Scotland, but of late years the Ring.Dove has increased in numbers till in certain counties its depredations have become a source of serious menace to the farmer. In Berwickshire some years ago a systematic raid was made upon these birds, resulting in the destruction of something over roo, 000 in one month. As their chief food consists of peas, seeds, and grain, there is little doubt that when their numbers become excessive the agriculturalist suffers, even though it is taken into consideration that they also consume the seeds of noxious weeds.

In autumn the favourite food of the Cushet.Doo is the beech mast on which they feed to repletion, and good sport may be obtained by hiding up in the vicinity of a beech wood at this season of the year. The length of the Ring.Dove is some 16 inches, its colour is slaty blue, washed with a vinous tinge, and neck and breast possess the rich metallic lustre common to the Dove tribe. On either side of the neck is a large spot of white feathers, from which it derives its rame of Ring. Dove. The hill and feet are of a hright coraal red. 
'he Ring. Move in its usual haunt is a shy and wary hird, it is more frecuently heard than seen. Through the spring and summer its low sueet cronning love song niay be heard in almost any wood. land, wlile its noisy departure when startled giver one the idea of it almost if ting through the overhead folizge. Sirangely enough this bird list of recent years become a denizen of the parks of London, and the cusutryman is astonished to see bis llative Cushat, usually so shy and waty, liere talle and confiding, so much so, as to actually take food from the outstretched hand

The nest of the Riug. Dove is frequently placed on the branch of a fir tree though it will select ivy covered irees and various other sites. It is built rudely of a few dried twigs, and is frequently so loosely constructed as to show from below, through the interstices of its frame, the two white eggs which it contains.

Rook. - The Rook, often erroneously called the Crow, is one of the most familiar and widely distributed of Britisb birds. It is to be foundeverywhere, save in the outer islands, and even to those spots is occasionally a visitor. The plumage of the Kook, as seen in the sunshine of early spring, has ricli dark blue gloss; the length of the bird is seventeen inches, and in the mature form, the face is devoid of feathers, giving it an unsightly and almost repulsive look. As is well known, the Rook is gregarious, both in winter and during the nestiug season, which begins early in March, at which time they build their nests in colonies, known as rookeries. In the main the rook is undoubtedly the farmer's friend, ridding bis ground of the grubs of many destructive insects. Tlie eggs are three to five in number, pale green in ground colour, spotted and blotched with blackish brown.

Snipe, Common.-In size the Common Snipe occupies an inter. mediate place between the Great Snipe and the Jack Snipe. Its length is some ten inches, and its chicf characteristic is its long hill, which measures about three inches, or not far short of one.third of its total length. When in flight it appears to be a dark brown hird, but on close examination its plumage is found to he of a rich brown, each feather centred with glossy black, and in certain parts, particu. larly the tail coverts and tail itself, the colour is exquisitely delicate and beautiful. The eye of the Snipe is placed very far back on the head, and this is no douht to enable the bird to freely indulge in the probing of the soft mud of river bank and pool edge, which is the means by which it finds its food. This consists chielly of worms, in the capture of which it is guided by a delicate and wonderfully con. structed nerve which extends down the whole length of its hill.

The Snipe is resident in our Isles, and breeds in many counties, chiefly in Ireland and in Scotland. Its numbers are, however, enormously inereased in winter when au invasion from north and east takes place to our shores. It is a bird much valued by the sports. man, more on account of the difficulty of bagging $i t$, than for its edible properties, the bird being so small. When the Snipe is flushed it rises with a shrill cry of "Scape," "Scape." in rapid zigzags until at a litule distance from the intruder, when it makes of in a straight line at high speed. The nest of the Snipe is placed upon the grouni, usually in a tuft of grass or sedge, the depression is lined with grass, and contains four pear-shaped eggs, often very hand. some, the ground colour stone spotted and hlotched with rich hlack.

Sparrow-Hawk. - This bold raptorial birl is resident with us during the year, and despite the movt persistent persecution of the species hy the ganie-keeper over the length and breadth of the land, 
still manages to maintain itself in considerable numbers. The female is, as throughout the raptorial family, considerably larger than the male, and it is unfortunately unquestionable that she destroys, especially at the breeding season, numbers of the young of the game birds near whose haunts she invariably nests. The site selected by the Sparrow-Hawk for nesting purposes, is frequently a corner of a wood or on the edge of a glade inside a wood, and so suitahle a position does this site appear to be that pair after pair of Sparrou. Hawks may be shot from the nest only to be replaced each succeeding year by another couple. The male is hlue-black above, the hreast white suffused with hrown, harred with a darker shade of brown, while his mate is ratber lighter in colour, the breast white harred with ashy grey. The legs of hoth are yellow, with toes greatly developed, giving great grasping power, and ending in needle. pointed talons. The eggs, five or six in number, are of hluish-white ground colour, richly hlotched and marked with reddish-brown.

Starling.-This bird is familiar to all, though at one time it was a comparatively rare hird, particularly in Scotland, where Sir Walter Scott, it is recorded, rode 30 miles to see a nest of young, so uncommon was this hird in his day. Tlurough whatever cause, it is unquestionable that the number of Starlings in Britain, where it is a resident and widely distrihuted lreeding hird, within recent years has greatly increased. In addition to our own native birds, enormous numbers of Starlings fock to our shores in the autumn months, and one of the most wonderful and interesting sights in bird life is to be witnessed, when in the darkening bour of an autumn afternoon one waits near one of their roosting places, from every point of the compass at short intervals single birds, little hunches, larger companies, and great fincks may be scen winging their way to the shelter where they pass the night. The writer has on more than one occasion witnessed the gathering of these birds, comprising hundreds of thousands, in one comparatively small shruhbery, where their whistling and cluattering resembled the noise of many waters. Before fually settling these hirds rise again and again into the air, wheel round several times, and then pour then. selves down from on high into the hushes hefore they finally settle to rest for the night, and to see these countless thousands of hirds wheel round, then suddenly turn, almost at right angles, as though animated by one single impulse, is suggestive of some strange understanding between the units, which can enable the mass to act so simultaneously.

The Starling is some eight inches in length, and is of a dark blue colour, waslied with brilliant metallic green and blue lustre, though a grey spot at the point of each feather suhdues this brilliance and imparts a brownish tinge. It is an indefatigable worker, and may be seen in pairs or small family parties ohtaining its food, which consists of slugs, small worms, grubs, and various forms of iusect life, by carefully examining almost every individual inch of lawn and pasture over which it runs with hurried eager steps.

The Starling huilds its loosely-constructed nest, consisting of a few straws, in holes, frequently in hollow trees, but in all sorts of situations, such as lioles in walls, drain pipes, in chimneys or corners of the ronfs of sheds and byres, while a favourite situation is in holes in the thatch of an old cottafe. The eggs are of an exquisite and delicate pale blue, unspotted, and number from five to eight, or even nine, thougl it lias been suggested that in the case of numbers, such 25 the last, thev are the product of two hens. 
Tern, Arctic.-This hird is not to be distinguished from the Common Tern save by the erjert, and, indeed, it takes a very exact knowledge for even the student 10 determine between the Cornmon and Arctic l'erı. The latter is very slightly smaller in size, being, perhaps, only lialf an inch less in length, but in general colour, appearance, and hal,it it in no way differs from its congener. The one infallible test lies in the difference of the lengeh of the tarsus, which in the Arctic "lern in longer than in the Coinmon lern.

I'lie Arctic T'ern is the only species found nesting in the Sliet. lands, where it breeds in large colonies on some of the out lying skerries. Fut ther south it is not uncoum non to find colonies of both Arctic and Coumon Tern breeding in close proximity. Generally speaking, those nests nearest the actual shore belong to the Arctic, the Common 'J'ern not infrequently occupying a site farther from the sea, often in swampy parts or amongst the sandy dunes. The eggs of the Arctic 'lern are two in $111 \mathrm{mber}$, very seltom three, and resemble closely those of the Conmon Tern, though, if distinc. tion can be made, they are possilly a trifle smaller and more boldly niarked, though the eggs of each species run through so many variations as to ynite overlap each otlier in this regard. The Arctic Tern is, like the Common Tern, a bird of very powerful Ilight, which it can sustain bour after lonur unweariedly. It also is 2 summer visitant to ottr shores, and during the winter season dis. tributes itself along the shores of Aftica and further east.

Tern, Common.-This species is 2 summer migrant to our shores, along which it is wiclely distributed. It does not extend to the Shetlands and in the Orkneyss and the extreme north of Scotland it is outnumbered by the Arctic Tern, but it is the more numerous along the sotuthern parts of the Scottisly const and out the coasts of England.

The Comnon Tern is all elegant bird in regard to shape, and its plumage is delicately coloured. It is known as the Sea Swallow, and owes this name to its resemblance in shape to the latter bird. 11 is, however, nuch larger, being some 85 inches in length, and in colour it is above of pearly grey, the head being capped with hl ock, bill and feet of brilliant blood red, and it is only owing to the length of the wing and the long forked tail that any reseniblance can be traced entitling it to the nanie of Sea Swallow. The Tern in its manner of obtaining food comes between the diving sea fowl, such as the Gannet, and the surface feeders, sucb as the Gulls. It feeds chieby. on the fry of fish, and in pursuit of these passe; with hovering flight along the shore, its liead downturned with beak at right angles to its breast, every now and again darting from above and striking the water in a clond of spray, but it at no time appears to descend more than a few inclies below the surface. The Common Tern breeds in colonies, always near water and usually on the sea coast, a favourite site being a low.lying islet. Uccasionally these colonies are very large, the nests usually placed in such close proximity as to make it difícult for a visitor to avoid stepping on the eggs, while the ear is assailed by the ceaseless cry, resembling $k \cdot \pi r$, shrill and long drawn out, of the birds disturbed from their nests. The intrusion is vehemently resented by many a downward swoop towards the tisitor's head. These birds seen to get along with little sleep, as a visit to their nestiug place at any hour of day or nizht reveals the same restless clamour. The nest of the Common Tern is placed upon the ground. The eggs are two or tbree, frequently three, in number, and are of a stone or olive ground colour, spotted with black, with fainter under shell markings of a purplish tinge. 
Tern, Sandwicl. - This is tbe largest of the genus which visits our shores, to which it is a summer migrant. It is also of stouter build than the two earlier-mentioned species, and its hill is stronger and of beavier make. In colour it resembles the Common Tern, hut is rather darker, while the bill and feet, instead of being of the brilliant red of the Common and Arctic Tern, are black, the bill tipped with yellowish. The black cap of the Sandwich Tern is elongated, the bindmost feathers forming what might he almost termed 2 crest. At one time this hird visited our shores in con. siderable numbers, breeding in colonies at various parts, but unfortunately it is no longer to be found in such large numbers, though at various places carefully guarded colonies still exist, and are, indeed, in certain places increasing in numbers. In hahit the Sandwich Tern resemhles its smaller cousins, feeding on the fry of fish which it captures in a similar way. The eggs of the Sandwich Tern are to be found at the end of May or in early June. They are placed on the ground in a slight plate-like depression, sometimes entirely unlined, sometimes lined with a few pieces of grass. The eggs are two and three in number, usually two, and are very rich and handsome in appearance. The ground colour varies from stone to buff, and they are ricbly spotted witb black, with strong purplish brown under-markings. A favourite site for the hreeding-place of the Sandwich Tern is on an island. There is considerable colony on one of the Farne Isles, wbere the birds are carefully protected.

Titmouse, Long-tailed. - This feathered mite is a resident with us, widely distributed, and in certain districts fairly coinmon. As will be seen in the illustration it is possessed of a tail longer than its hody, which is of a dull whitish-brown colour, sligbtly suffused with pale rose. It is indeed a charming sight, to see the little family, consisting of tbe two parent hirds, with their six, eight, nine, or even ten young, flitting along from tree to tree as is their habit in the autumn montbs. Their tiny size combined with their length of tail renders them quite distinct from any of our other birds. The Long tailed Tit huilds what is unquestionably the most beautiful of British nests, an elongated structure of moss thickly covered with lichen, and felted together with cohwebs, with a small aperture beneath the upper end, and cosily lined with feathers. One patient observer took the trouble to ccunt the number of feathers contained in one nest, to find the surprising number of over 2000 . Tbe eggs, usually ten or eleven in number, are extremely small, pure white, and spotted with tiny dots of light brown. With the exception of the Golden.Crested Wren, the egg of the Long-tailed Tit is the smallest laid by any of our British birds. The instinct is surely a marvellous one, which enables these feathered mites to feed without favour and in due turn tbe ten hungry tots contained in their cradle.

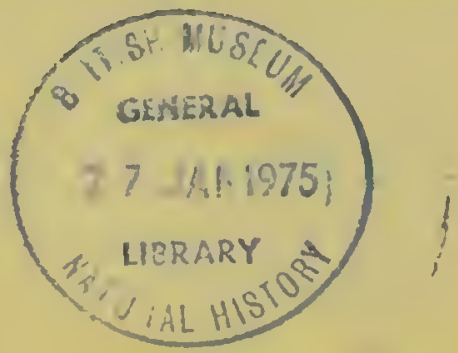




\section{Gowans's Books for the $Y_{\text {oung }}$}

A SERIES of prettily got up little volumes containing old lavourites and other bocks sure to become tavourites.

\section{ILLUSTRATED EDITION}

NO. I. THE SEVEN CHAMPIONS UF CHRISTENDOM. By W. H. G. Kingston. With sixteen coloured illustrations by Siephen Reid.

No. 2.-THE HEROES. Greck Fiairy Tales for my Children, by Charles Kingsley. With sixteen coloured illustrations by Charles Pears.

Price: Art Cloth Boards, I/- net. Postage, Ifd, each.

\section{ORDINARY EDITION}

No. I. THE SEVEN CHAMPIONS OF CHRISTEN. DOM, By W. H. G. Kingston.

No. 2.-THE HEROES. Greek Fairy Tales for my Children, by Charles Kingsley.

No. 3.-THE STORY OF PRINCE AHMED AND THE FAIRY PERIE BANOU (from the Arabian Nights).

Price, Picture Boards, Cloth Back, 6d, net., Postage, Itd. each.

Gowans \& Gray, Ltd., London and Glasgow 


\section{The Children's Bookshelf}

PRETTY little Books with about a dozen coloured lliustrations by well-known artists.

No. x.-THE UGLY DUCKLING, by Hans Andersen. Coloured illustrations by Stewart Orr.

No. 2.- LITTLE KLAUS AND BIG KLAUS, by Hans Andersen. Coloured illustrations by Charles Pears.

No. 3.-THE PIED PIPER OF HAMELIN, by Robert Browning. Coloured illustrations by Charles Robinson.

No. 4. -THE STORY OF CHANTICLEER. Re-told from Chaucer, by R. Brimley Johnson. Coloured illustrations by Stewart Orr.

Price, 60. each in Parchment Covers. Polt freg 70.

GOWANS \& GRAY, Ltd., London \& Glasgow 


\section{Gowans's Nursery Pictures}

$T^{H E}$ publisher, believe that the complete novelty of a series of four coloured pictures by Mr. Stewart Orr, which they have just published will ensure their immediate success. No sinilar series is in existence, and the attractive appearance of the prints will delight every child who is presented with them. They are supplied in meat brown paper frames, ready to bang up, at Sixpence each, net, Postage, threepence extra.

THE FOUR SUNECTS ARE:

\section{OLD MOTHER GOOSE} HUMPTY-DUMPTY

\section{TOM THE PIPER'S SON}

\section{THREE BLIND MICE}

The pictures are to be had through any bookseller, printseller, or stationer, or direct from the publishers,

GOWANS \& GRAY, Lid, 58 Cadogan St, Glasgow 


\section{Gowans's Colour Prints}

In Germany during the last tew years attempts have been made by some enterprising publishers to raise the standard of artistic taste of ordinary people with little money to spend in pictures, by issuing colour print for wall-decoration, specially painted by the best artists in the country, at very cheap prices. These attempts have been attended with great success, and many hundreds of such prints can now be had. Messrs. Gowans \& Gray have gone a step further still, and they have now issued the first three of a similar specially-painted series of pictures, $14^{\prime \prime} \times 10^{\prime \prime}$ in size, which are sold in neat brown or white paper frames, ready to bang on the quall, at the unprecedented price of One Shilling each ; post free, $\mathrm{r} / 4$.

THE TITLES OF THE THRFE ISSUED ARE-

No 1. "MY POOR DOG TRAY," by Chakles Pears.

No. 2. "APPLE BLOSSOM," вY Jessir M. Kung. No. 3. "FAR FROM THE MADDING CROWD," BY STEPIIEN REID

They are all artistic enough to hang in any roon, no matter how luxurious, and should supersede those oil-colour daubs and grocer's-calendar-pictures, which are so often to be seen hung up in British houses, where everything else, except the pictures, is in good taste.

Gowans \& Gray, Ltd., London and Glasgow 
$\cdot$ 
